University of Redlands

\title{
A Facilities Management Solution for Montessori in Redlands
}

\author{
A Major Individual Project submitted in partial satisfaction of the requirements \\ for the degree of Master of Science in Geographic Information Systems \\ by \\ William Mertens \\ Mark Kumler, Ph.D., Committee Chair \\ Douglas Flewelling, Ph.D.
}

December 2021 
A Facilities Management Solution for Montessori in Redlands

Copyright @ 2021

by

William Mertens 
The report of William Mertens is approved.

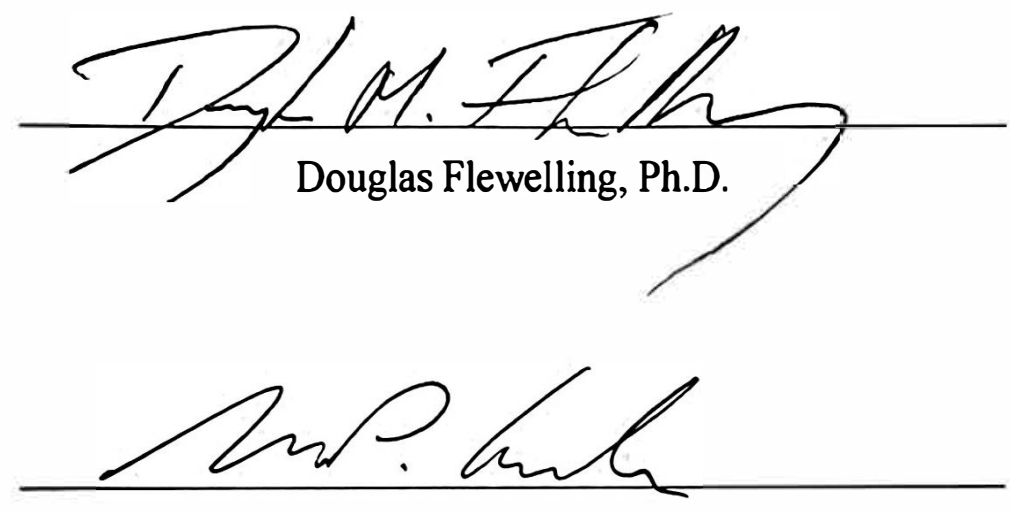

Mark Kumler, Ph.D., Committee Chair

December 2021 



\section{Acknowledgements}

To say I have done anything on my own would be a lie. I could not have arrived where I am without the countless people who want me to succeed and who supported me along the way. I'm going to thank a few of them here.

I would like to thank my advisor Dr. Mark Kumler for his help throughout this project. Your undying and patient support was invaluable in keeping myself sane and on track to complete this MIP on schedule. Thanks also to my other committee member Dr. Douglas Flewelling not only for agreeing to serve on my committee but also for kindly and calmly forcing me to think conceptually as well as practically. Thank you to the other GIS faculty members Dr. Ruijin Ma and Dr. Fang Ren and to adjunct faculty Jonathan Quinn, Makram Murad-Al-shaikh, Pinde Fu, and Kenneth Baloun for providing me with all the GIS knowledge I could ask for. Thank you, Andrea Alvarado, for being supportive and helpful to all of us. Thank you, Lisa Benvenuti, for helping me work through issues and assisting with all the metadata. Thank you, Bridgette Callahan, for helping me edit and revise. Creating a productive learning environment remotely is a challenge. These instructors and staff managed to pull it off beautifully.

A big, enormous, massive thanks and congrats goes to my fellow members of cohort 39, 39.5, and 37.5. Jordan, Stephanie, Edmond, Courtney, Jessica, Tim, Tanessa, Aubri, and Faraz: we met remotely, but I'm thrilled we all eventually were united in person. We supported each other through this difficult undertaking and you all should be enormously proud of yourselves. Thanks especially to Faraz, who assisted with getting my Indoors data just right. A big thank you also goes to Aubri, who helped me through some particularly challenging issues.

Thank you to Phil Sanchez, who generously donated his time to help with digitizing and enabled all the buildings on the MIR campus to be included in the GIS. Not only is digitizing mind-numbingly boring, it's also massively frustrating, so to be willing to donate as much time as you did was truly a gift to me, this project, and MIR.

To my former professors at Beloit College, especially Dr. Sue Swanson, Dr. Leslie Williams, and Dr. Kylie Quave: Thank you for all the support throughout my undergrad. Thank you, Kylie, for inspiring me to study anthropology and being endlessly supportive in those first two years. Thank you, Leslie, for making me a better writer. Thank you, Sue, for showing me that GIS is awesome.

To my Beloit friends Kerry, Claire, and Mustafa: I love you all very much and you have no idea how much it means that you are in my life. I missed you all this year.

Lastly, thank you to my family who have always and will always support me. Thank you, mom, for listening to me whine about bugs in ArcGIS Pro for an entire year. Thank you, dad, for unconditional support and being my first role model. To my sister Brooke: you are a kind, smart, and beautiful person. We somehow manage to simultaneously think incredibly alike and diametrically opposed, but I could not imagine growing up without you. Family, I love you all. 



\begin{abstract}
A Facilities Management Solution for Montessori in Redlands

by

William Mertens
\end{abstract}

\begin{abstract}
Montessori in Redlands is a small, private, non-profit Montessori school in Redlands, CA serving toddlers to pre-teens. Their campus includes six buildings nestled in grapefruit groves and spread across twelve acres. The school requested assistance implementing a solution for managing these facilities and their contents. Staff members identified three problems of high concern: (a) lost classroom items due to lack of inventory tracking, (b) unexpected costs to replace failing building components, and (c) emergency first responders lacking access to high-quality campus maps. This project implemented a GIS to create a facilities management solution to address these concerns and allow for expansion in the future. Utilizing ArcGIS Online, the campus data are stored in a series of Hosted Feature Layers and are manipulated in Web Maps, Web Apps, Dashboards, and the Field Maps mobile application. Within these layers are building floorplans that use the ArcGIS Indoors information model to dynamically store and query indoor features in a way that makes sense to the user. All features "know" where they are in relation to others so users can locate items between buildings and floors without having to interact with the raw data. Indeed, ease of use was considered one of the most important aspects of the system. Consequently, the implemented system can be accessed, adjusted, and expanded without touching ArcGIS Pro.
\end{abstract}





\section{Table of Contents}

Chapter 1 - Introduction ................................................................................................. 1

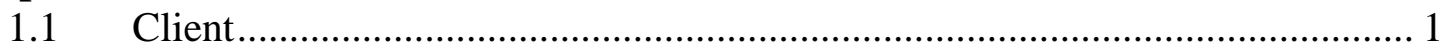

$1.2 \quad$ Problem Statement ........................................................................... 1

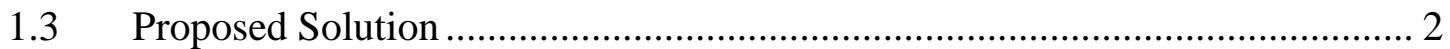

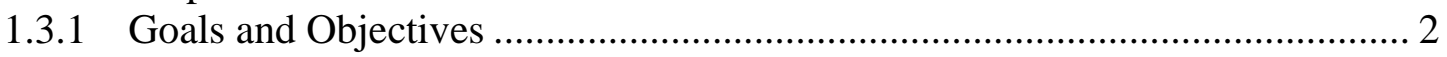

1.3.2 Scope

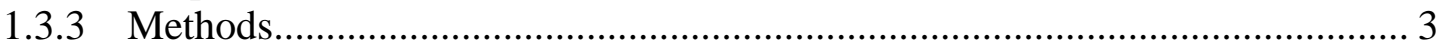

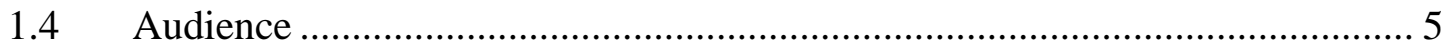

1.5 Overview of the Rest of this Report .............................................................. 5

Chapter 2 - Background and Literature Review ............................................................ 7

$2.1 \quad$ Overview of Facilities Management......................................................... 7

2.1.1 Differing Approaches to FM................................................................... 7

2.1.2 FM in Schools and the Montessori Perspective ............................................. 9

2.2 GIS for Facilities Management ................................................................ 10

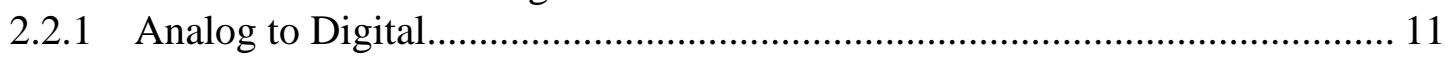

2.2.2 Georeferencing Scanned Floorplans .......................................................... 12

2.2.3 Digitization and Data Models ................................................................... 12

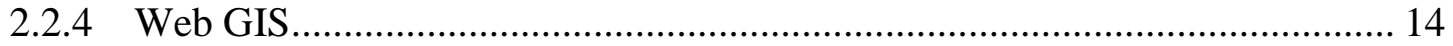

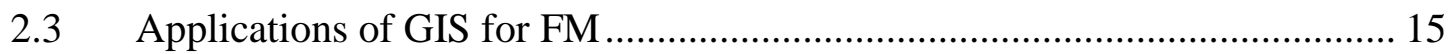

2.3.1 Digitizing and Map Creation ………………….......................................... 15

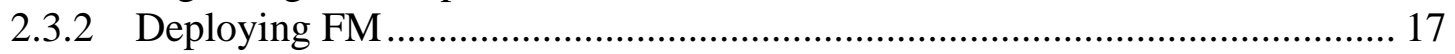

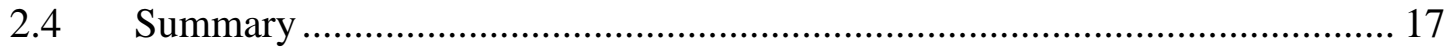

Chapter 3 - Systems Analysis and Design....................................................................... 19

$3.1 \quad$ Problem Statement .......................................................................... 19

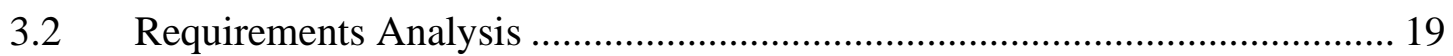

3.2.1 Functional Requirements ..................................................................... 19

3.2.2 Non-functional Requirements ................................................................ 21

3.3 System Design ............................................................................... 22

3.3.1 Data Creation and Editing with ArcGIS Pro.................................................. 22

3.3.2 The ArcGIS Online System ................................................................ 23

$3.4 \quad$ Project Plan ...................................................................................... 23

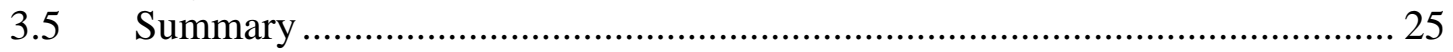

Chapter 4 - Database Design.............................................................................................. 27

4.1 Conceptual Data Model .......................................................................... 27

4.2 Logical Data Model …………………………................................ 28

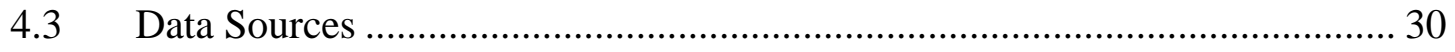

4.4 Data Collection Methods ........................................................................... 31

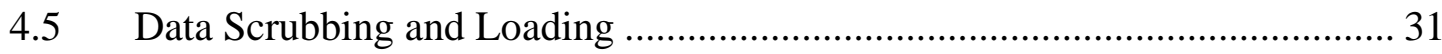

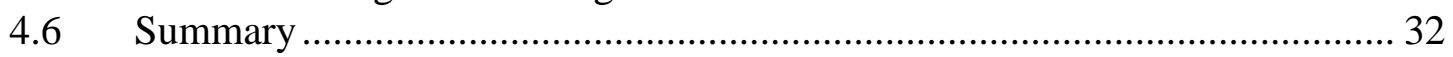

Chapter 5 - Operations in Desktop Software ..................................................................... 33

$5.1 \quad$ Preparing and Processing Paper Floorplans................................................. 33

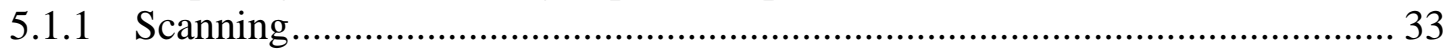




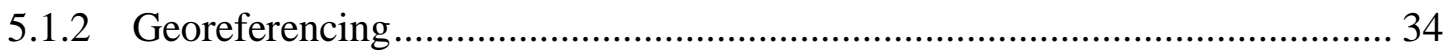

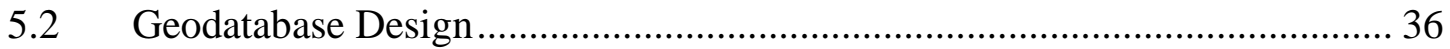

5.2.1 Fundamental ArcGIS Indoors Feature Classes ........................................... 36

5.2.2 Facilities Management Feature Classes …………………………………..... 37

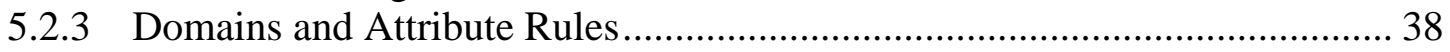

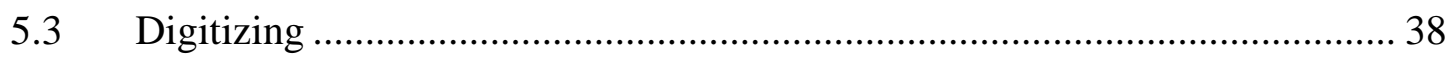

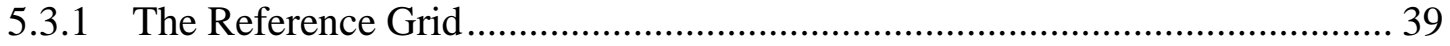

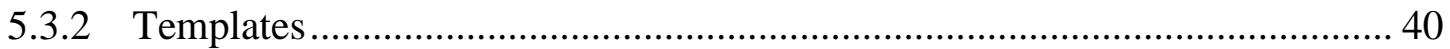

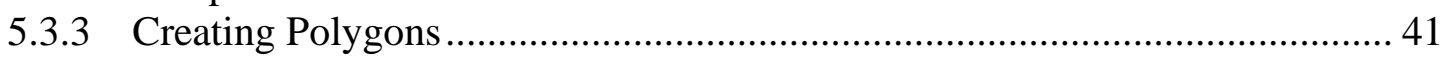

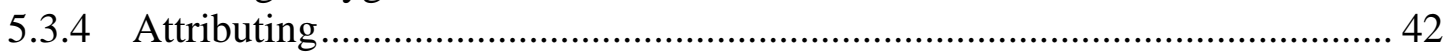

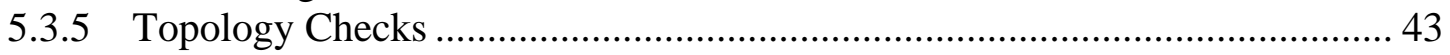

5.3.6 Creating and Attributing Facilities Management Features ............................. 44

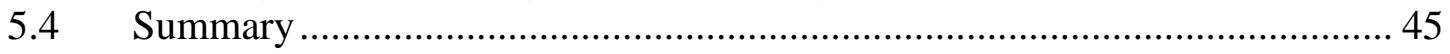

Chapter 6 - Online Deployment............................................................................... 47

6.1 Maps and Apps in ArcGIS Online ............................................................. 47

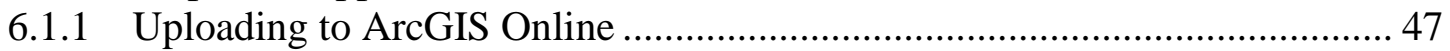

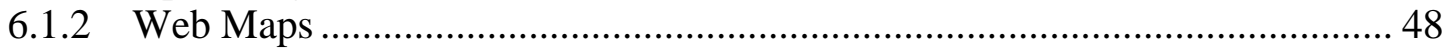

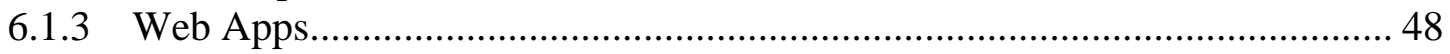

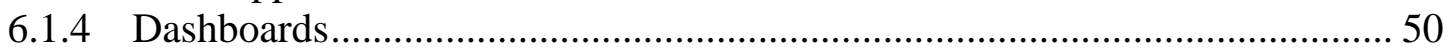

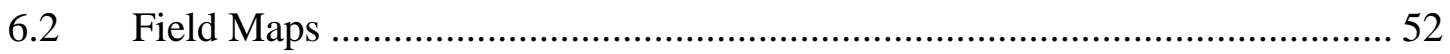

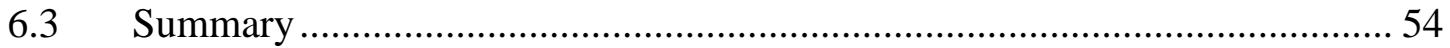

Chapter 7 - Results and Analysis.................................................................................... 55

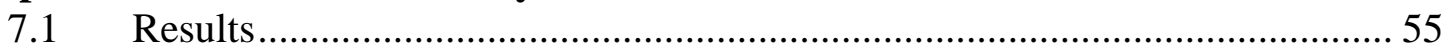

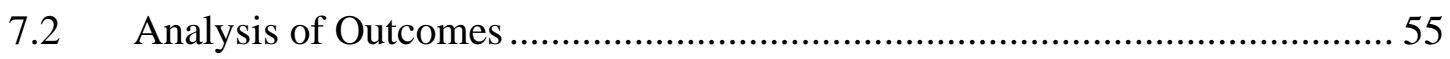

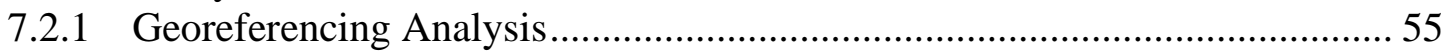

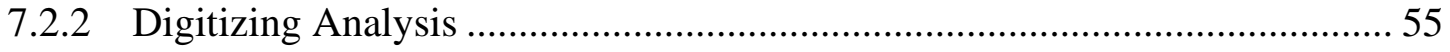

7.2.3 Analysis of Uploading to ArcGIS Online .................................................... 56

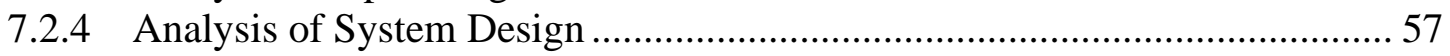

7.2.5 Analysis of System Construction …………………................................... 58

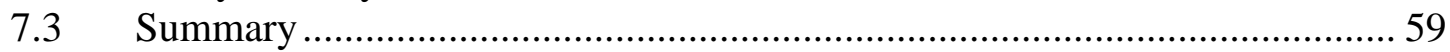

Chapter 8 - Conclusions and Future Work .............................................................. 60

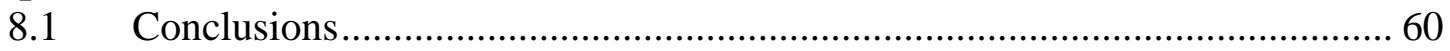

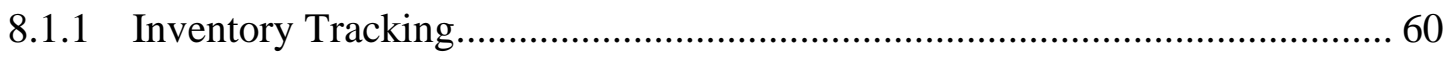

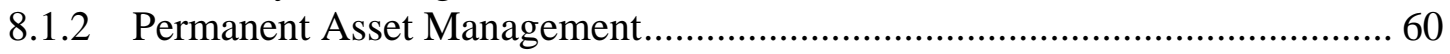

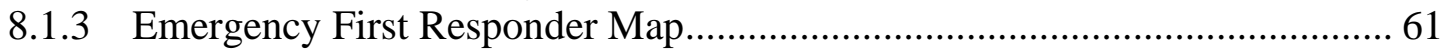

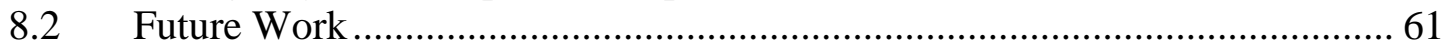

8.2.1 Expanding ArcGIS Indoors Functionality ......................................................... 61

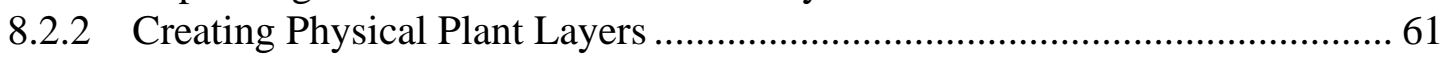

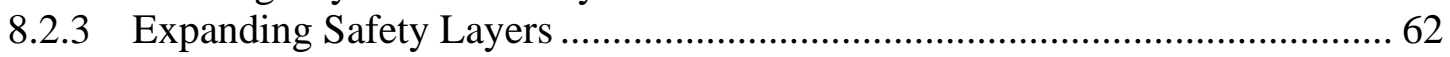

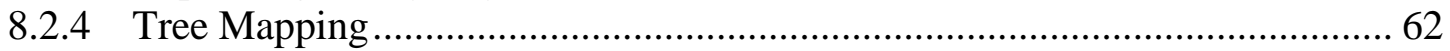

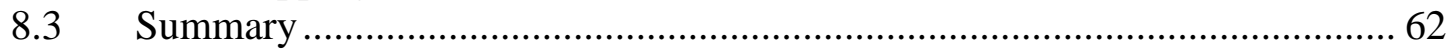

Works Cited ............................................................................................................................ 63

Appendix A. Schema Report......................................................................................... 67

Appendix B. Campus Maps ...................................................................................................... 79 
Appendix C. Custom Symbology ......................................................................................... 93

Appendix D. Online System Components.............................................................. 95 



\section{Table of Figures}

Figure 1-1: List of Tasks and Project Phases........................................................... 4

Figure 2-1: IndoorGML Data model (from Tekavec \& Lisec, 2020) ........................ 13

Figure 2-2: Example of a building floorplan (from Bahri et al., 2019) ...................... 16

Figure 2-3: Various image-parsing methods (adapted from Dodge et al., 2017) ...... 16

Figure 3-1: System Architecture ........................................................................22

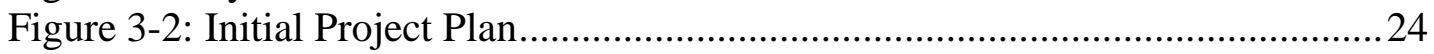

Figure 3-3: Updated Project Plan....................................................................... 25

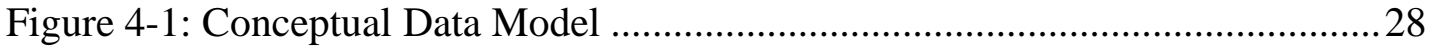

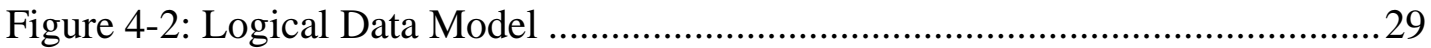

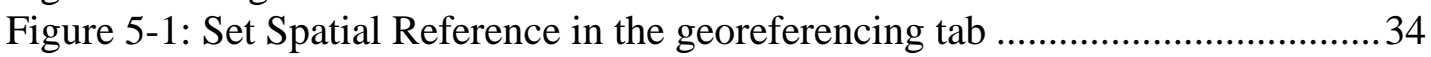

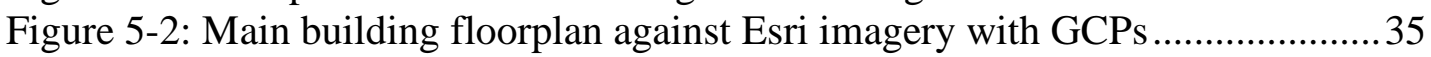

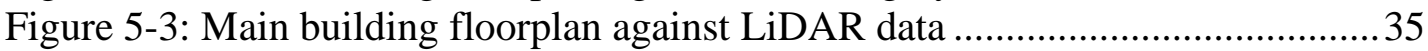

Figure 5-4: Section of the Units attribute table showing IDs for Indoors ................. 37

Figure 5-5: Reality versus floorplan depiction indicating removed fixtures ..............39

Figure 5-6: The reference grid in the Northeast corner of Building A ......................40

Figure 5-7: Configuring the window group template .......................................... 40

Figure 5-8: Creating door features using a preset template ................................... 41

Figure 5-9: Drawing a Unit polygon in the main building ......................................42

Figure 5-10: "Details Use" coded value domain .................................................. 43

Figure 5-11: Digitizing errors omitted from topology checks ............................... 44

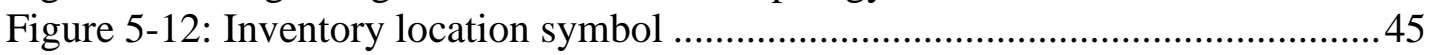

Figure 6-1: Floor filter in the Inventory Web App. Set to level 1 ...........................49

Figure 6-2: Viewing inventory locations and their associated items ........................50

Figure 6-3: Category selectors with floor picker dropdown selected ........................50

Figure 6-4: Three elements below the map frame in the Assets dashboard ...............51

Figure 6-5: Safety Dashboard showing Building A...........................................51

Figure 6-6: Field Maps showing Safety (Building A level 1 and 2).......................52

Figure 6-7: Field Maps configurator editing Inventory Items ...............................53

Figure 6-8: Inventory location, related items, and item details ...............................54

Figure 7-1: Section of Main Building office and kitchen area .................................56

Figure 7-2: Rendering in ArcGIS Online (top) vs. ArcGIS Pro (bottom) .................59 



\section{List of Tables}

Table 1. Task Breakdown ................................................................................. 3

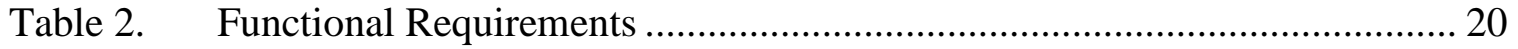

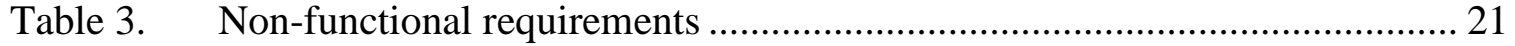





\section{List of Acronyms and Definitions}

$\begin{array}{ll}\text { API } & \text { Application Programming Interface } \\ \text { BIM } & \text { Building Information Modeling } \\ \text { BLE } & \text { Bluetooth Low Energy } \\ \text { CAD } & \text { Computer Aided Design } \\ \text { DPI } & \text { Dots Per Inch } \\ \text {.dwg } & \text { Proprietary file format for the Autodesk product suite } \\ \text { Esri } & \text { Environmental Systems Research Institute } \\ \text { FC } & \text { Feature Class } \\ \text { FD } & \text { Feature Dataset } \\ \text { FM } & \text { Facilities Management } \\ \text { GCP } & \text { Ground Control Point } \\ \text { GIS } & \text { Geographic Information System } \\ \text { GML } & \text { Geography Markup Language } \\ \text { GPS } & \text { Global Positioning System } \\ \text { HVAC } & \text { Heating, Ventilation, and Air Conditioning } \\ \text { IFMA } & \text { International Facilities Management Association } \\ \text { LiDAR } & \text { Light Detection and Ranging } \\ \text { MIR } & \text { Montessori in Redlands } \\ \text { OCR } & \text { Optical Character Recognition } \\ \text { SPCS } & \text { State Plane Coordinate System } \\ \text {.svg } & \text { Scalable Vector Graphics file extension } \\ \text { tif } & \text { Extension for Tag Image File Format files } \\ \text { UI } & \text { User Interface } \\ \text { VGI } & \text { Volunteered Geographic Information } \\ \text {.xml } & \text { Extensible Markup Language file extension } \\ \text {.zip } & \text { File extension. Container format for compressed files } \\ & \\ \end{array}$





\section{Chapter 1 - Introduction}

The Montessori in Redlands (MIR) school requested assistance implementing a facilities management (FM) platform for its campus using a geographic information system. FM is a broad term, but generally refers to the management of maintenance and use of the physical assets of an institution (Kamaruzzaman \& Zawawi, 2010). A well-designed, well-maintained facilities management platform can enable a "one-stop" solution for every-day operations and long-term planning. In many cases, organizations rely on decentralized and fragmented information to manage facilities. A GIS-based approach to FM would represent buildings, grounds, and their attributes spatially and establish a modeled relationship unique to GIS.

For this implementation, the needs of MIR were to (a) track inventory of classroom materials, (b) manage long-term asset maintenance and repair, and (c) provide emergency first responders with a detailed basemap of campus. The GIS implementation will also have applications in other environments, but these were the areas of most need identified by the client. This paper addresses the aforementioned problems and presents a solution using GIS technology to assist MIR in the management of their facilities.

\subsection{Client}

Montessori in Redlands (MIR) is an independent, non-profit school in Redlands, California which serves about 360 students from pre-K to $6^{\text {th }}$ grade (Montessori In Redlands, 2020). There are six buildings on campus; five are used by MIR and one is used by Grove School, which serves the $7^{\text {th }}-12^{\text {th }}$ grades. Ellen Camarillo and Lisa Kensok served as points of contact for this project. Ellen Camarillo is the facilities coordinator and Lisa Kensok is the marketing and communications director. Their role was to provide all campus data, provide feedback, and define the final scope of the project. Ellen Camarillo played an integral part in this project, providing access to the school grounds, answering questions, and promptly returning valuable feedback.

In general, the staff at MIR have limited knowledge of GIS, yet were able to define general requirements and their priority. Technical requirements were derived from these conceptual requirements. This limited knowledge also highlights the need for a GIS that is simple to use, does not require routine maintenance through specialized software, and can be accessed through a mobile device. More extensive maintenance and support can be provided by MIR parents who work for the Environmental Systems Research Institute (Esri). Two parents have already expressed interest in maintaining the project, including Phil Sanchez, a product engineer for Esri's digitizing team.

\subsection{Problem Statement}

MIR required an integrated facilities management platform which encompassed all relevant data for asset management, inventory management, and first responders. Further, the client articulated its need for the system to be centrally accessible to all members of the MIR staff and first responders. Completing the project presented both a capacity and 
a capability problem, in that MIR had neither the dedicated staff to implement FM or the necessary expertise to complete the project on their own.

Because building floorplans existed only in paper form, building information was largely not digitally accessible, much less centrally accessible. Due to these limitations, the school faced problems while implementing projects and conducting day-to-day business. Inventory was managed using pen and paper or not at all, and planning maintenance and replacement of school assets was conducted through spreadsheets. In addition, first responders did not have relevant information for the school such as building names or door numbers.

\subsection{Proposed Solution}

The proposed solution created a basemap of the MIR campus, including building interiors, as the basis for a FM platform. Implementing GIS for FM at MIR provided a common coordinate system for spatial data and enabled better tools for the organization of datasets. Datasets within the scope of this project were classroom inventory, asset management, and information for first responders. The system was designed to be easy to use, regardless of skill level, and be accessible with only a smartphone. The system also included the ability to update attributes without the use of specialized software. This section describes the methodology used to solve their problem.

The most important feature was the digitized building interiors, which was created from the original paper floorplans. These features were overlayed onto an existing basemap of the campus exterior, created by an Esri employee. The newly created campus basemap, now including buildings' interior features, can be used for future applications that are beyond the scope of this project.

\subsubsection{Goals and Objectives}

The goal of this project was to provide a FM solution at MIR by implementing GIS. Beyond providing a high-quality basemap of campus, the solution allowed MIR staff to manage classroom inventory and assist in long-range planning of school assets. A modified basemap was also made available to emergency first responders, enabling swift access to relevant location information such as door and room numbers. The schema also included the flexibility for future expansion and was implemented in such a way that requires little maintenance.

\subsubsection{Scope}

The main tasks of this project included digitizing floorplans in ArcGIS, creating the campus basemap, creating features and attributes for classroom inventory and asset management, publishing the data to ArcGIS Online, and deploying the tool in FieldMaps. Each of these tasks had to be completed before the next task could commence. Beginning the digitizing process required the delivery of the floorplans to be scanned. The project demanded approximately 500 to 600 hours of work. Table 1 shows the initial work breakdown structure for this project. 
Table 1. Task Breakdown

\begin{tabular}{|l|l|}
\hline Task & Time Duration (Hours) \\
\hline Requirements Verification & 20 \\
\hline Document Scanning & 10 \\
\hline Floorplan georeferencing & 5 \\
\hline Floorplan digitizing and map creation & 150 \\
\hline Creating FM features and attributes & 120 \\
\hline Publish to ArcGIS Online & 110 \\
\hline Deploy to FieldMaps & 90 \\
\hline Total & $\mathbf{5 0 5}$ \\
\hline
\end{tabular}

The time values listed in table 1 represent the minimum number of hours taken for each task. The most time-consuming aspect of this project was the digitizing. Since the buildings' interior features were stored only in paper floorplans, all the digitization had to be done by hand. The data schema chosen for this project also required each feature to include specific attributes to display correctly. These also had to be inputted by hand with the assistance of automated functions in ArcGIS Pro. Creating FM features was also a time-consuming process as the specific features desired by the client were not as clear as anticipated. This issue did, however, further highlight the need for a centralized FM solution at MIR. The specific outcomes of this project were as follows:

1. A geodatabase that includes feature classes for the ArcGIS Indoors data model.

2. Building interior features digitized from paper floorplans and stored in said feature classes.

3. Feature classes for classroom inventory and equipment maintenance. These serve as the basis for the FM platform.

4. Comprehensive campus map that includes both indoor and outdoor features.

5. A series of ArcGIS Online Web Maps, Web Apps, and Dashboards for classroom inventory, equipment maintenance, and emergency first responders.

6. FieldMaps deployment for accessing maps on a mobile device.

\subsubsection{Methods}

ArcGIS Pro 2.8 was used to complete all data creation and editing. The data were then published to ArcGIS Online, first to a University of Redlands account, then to an account owned by MIR. Licensing was provided through a donation from Esri and is hosted in the cloud. Accessing the platform requires only an updated web browser or a smartphone capable of running the most recent mobile applications. Backups of the data stored in a local file system were also provided to MIR.

Time constraints necessitated flexibility on some of the deliverables, including during digitization. None of the six buildings had Computer Aided Design (CAD) files accessible to the project, necessitating hand digitization. Given these time constraints, a staged methodology was used. This model combines the simplicity of the waterfall method and includes the ability to maintain flexibility such as with the spiral method. It was important to allow for the timely completion of certain workflows, but also to be responsive to changes as issues arose (Forsberg et al., 2005). One known issue was the 
availability of digitized CAD files, which were ultimately unavailable. This methodology considered that possibility and allowed for completion of only certain campus buildings. Figure 1-1 outlines the tasks and procedures involved in different project phases.

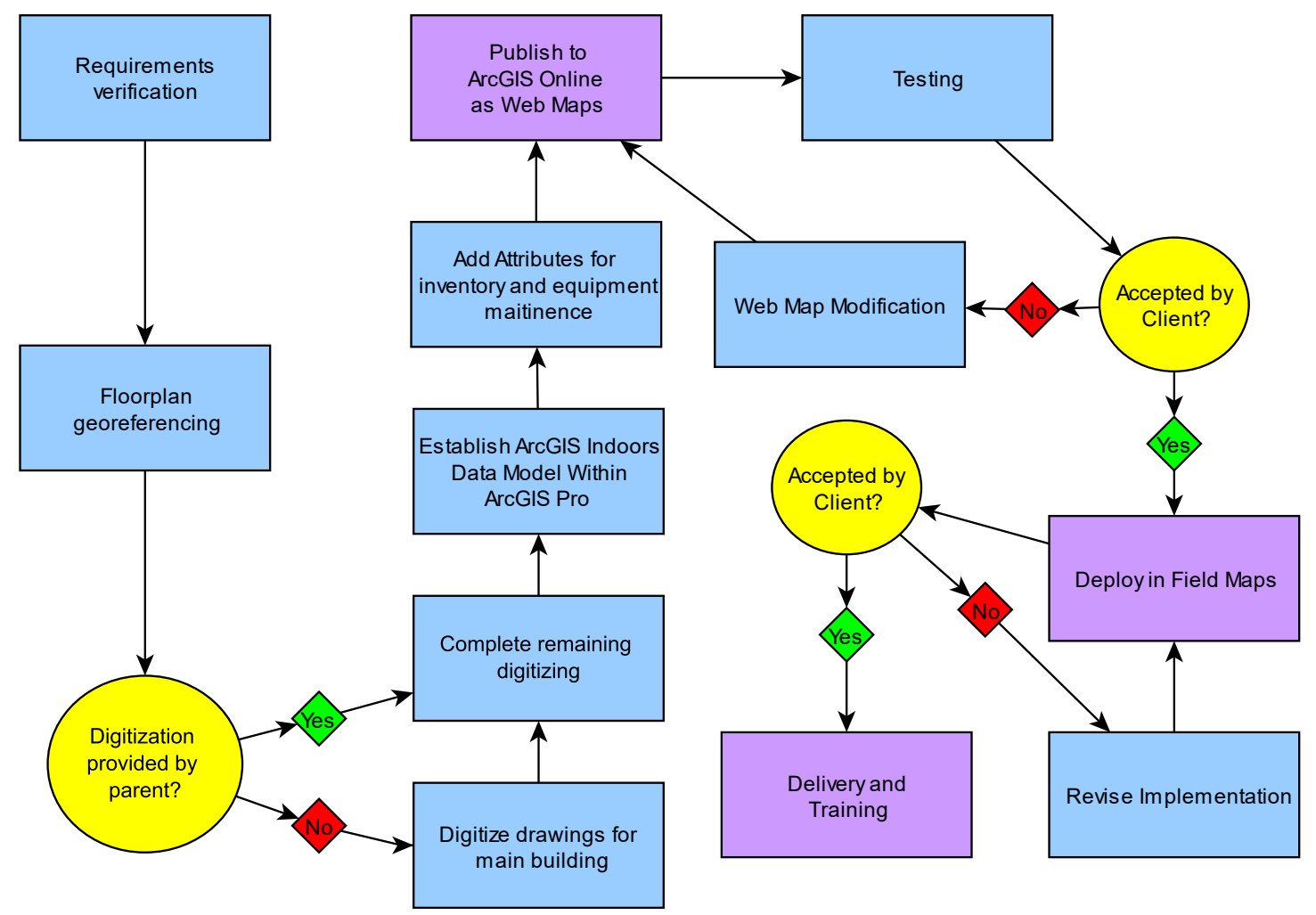

Figure 1-1: List of Tasks and Project Phases

Requirements verification included several preliminary meetings with the client to gather all relevant information and ensure all materials and data were available. These meetings also established the client's expectations for the project and cemented the role of the client in project completion. Client meetings also provided an opportunity to ensure digitizing assistance from MIR parents would be available. A project plan was then provided to the client for review and approval. The following tasks were established based on the results of these meetings.

The project commenced by scanning the paper floorplans into high-quality .tif files and by georeferencing in ArcGIS Pro using a combination of the Esri imagery layer and Lidar data for the Redlands area. A geodatabase was also created with separate feature classes based on the pre-defined ArcGIS Indoors model. These feature classes are viewable on a previously-created basemap of the campus exterior. This basemap was a few years old and needed to be updated to reflect recent changes.

After the floorplans were digitized and the Indoors model established, features were created to represent classroom inventory and asset management. These are represented by a points-of-interest layer. For inventory management, there are points for each classroom, with a related table listing the items they contain. Assets are also stored as point data and are placed at the location of the asset. Attributes include name, type, quantity, maintenance schedule, and replacement cost. 
Publishing to ArcGIS Online commenced only after the integrity of the geodatabase was fully verified. Three Hosted Feature Layers were published to the University of Redlands ArcGIS Online account and then moved to an account owned by MIR. The first layer was campus features for first responders, including door numbers. The second layer had inventory data for classrooms and was accessible to relevant staff and teachers and the third held the asset management data. Also included was the layer containing indoors information. These layers are capable of using the ArcGIS Floor Aware functionality included in ArcGIS Indoors, meaning that individual floors can be selected for viewing. The deployment to FieldMaps enabled MIR staff to access the platform on their mobile devices.

\subsection{Audience}

This project report is intended to be useful for both non-GIS and GIS users. The Montessori in Redlands staff are largely not GIS-literate. Therefore, this document provides a sense of understanding into how the system works and how it can be improved in the future. Several GIS projects have been conducted at MIR in the past, many of which included direct involvement from the students and staff. As such, the culture on campus creates a particularly welcoming environment for the implementation of GIS. It is, then, not the goal of this paper to sell the merits of GIS to the client themselves, but demonstrate the value of GIS to other organizations looking to implement FM.

For those who are GIS-literate, this paper includes best-practices for many of the major tasks in this project. It also outlines many of the problems and mistakes made along the way and how professionals completing a project like this can avoid them. In general, this report is useful to anyone who wants to digitize floorplans and implement floor-aware features, be it for facilities management or other applications.

\subsection{Overview of the Rest of this Report}

This project report is divided into eight chapters. Chapter two is the background literature review. It provides research into how GIS can be implemented to create a facilities management platform. It also highlights how emergency first responders utilize GIS for calls and best practices for digitizing. Chapter three discusses systems analysis and design, from how the requirements were gathered to how they were implemented into the system.

Chapter four is database design. In this chapter, the data models are described along with how the data were sourced, prepared, and cleaned. Chapters five and six discuss the implementation of the system. Chapter five explains data creation in the desktop software while chapter six examines the construction and operation of the online system. Chapter seven provides the results of the project and analyzes the final product, in comparison to the conceptual model. Chapter eight includes conclusions and recommends further work that can be completed. 



\section{Chapter 2 - Background and Literature Review}

The body of knowledge surrounding facilities management (FM) is immense. For example, the journal Facilities is dedicated to investigating how physical assets underpin the operation of organizations. The academic discussion on the topic of FM is underscored by disagreement on the role and future of the field. Most agree that FM is necessary, but the field has failed to reach a consensus on how it fits into the broader context of an organization. Dorian et al. (2012), for example, highlights public confusion about the role of FM and calls for FM professionals to solidify their understanding of the discipline. As such, this chapter will explore these definitional issues. Historically, FM is a relatively new field borne from the new style of offices that emerged in the mid- $20^{\text {th }}$ century. Understanding these underlying concepts is important to developing a system for FM.

GIS is one solution to FM and has been used in the past to provide a centralized system with a spatial context. Newer cloud GIS technologies have been recently improved to enable the creation of reliable and easy to use maps that are available across a wide range of devices. That is why this mapping project utilized web GIS to deliver valuable FM content to Montessori in Redlands (MIR). Web GIS maps are endlessly customizable, interactive, and accessible to almost anyone for important visuals and analysis.

\subsection{Overview of Facilities Management}

Facilities management is a broad term that can be applied to the maintenance, use, and operation of the physical assets of an institution, including the role of people in that operation. In addition to simply building management, Kamaruzzaman and Zarari (2010) see facilities management as a key factor in the success of an organization. FM, they say, should focus on "... raising the efficiency and sustainability of the management of space and other related assets management for people and processes" in addition to simply building management (p.75). In other words, the goal of FM is not just to improve the bottom line of an organization, it can also prove a valuable tool in fulfilling the goals of an organization. Governing bodies such as the International Facilities Management Association (IFMA) exist to provide certification, research, and news to FM professionals (Kamaruzzaman \& Zawawi, 2010).

\subsubsection{Differing Approaches to FM}

FM has a 40-50 year history, beginning in the United States, and emerged as a profession in the early 1980s (Duffy, 2000). According to the IFMA, at this time, a new style of office, the cubicle, was emerging, and those in charge of managing the new workspace were in need of direction. The Herman Miller Corporation, noted maker of office furniture, hosted a conference wherein key players in the move toward these new, more structured offices, known as systems furniture, laid the groundwork for the emergence of FM as a profession (IFMA, 2021a). Expansion into other avenues followed soon thereafter to potentially encompass the operation of all an institution's physical assets. 
The actual definition of FM remains ill-defined both as a body of knowledge and as a set of professional skills, often spurring arguments in the literature about the nature of the profession. FM's role in the core operation of an organization and the emphasis that should be placed on it is an ongoing subject of debate within the profession. Those in the role of promoting FM, like the IFMA, present an apocalyptic take, implying - or stating outright - that the success of an organization is entirely dependent on whether or not FM has been implemented (IFMA, 2021b). Of course, the associations which support FM professionals are interested in creating a narrative which convinces organizations of the merits of FM. Frances Duffy, who is considered among the pioneers of FM in the United Kingdom, argues "the way in which office space is managed through time is of equal significance to office design ... as software is to hardware, so facilities management is to design.” (Duffy, 2000, p. 371).

Duffy's argument - a good space is nothing without good people to run it - is compelling, although perhaps over-optimistic. Dorian et al. (2012), in an analysis of the differing definitions among the FM community, provides a less charitable take. They note that then-current definitions of facilities management could be summed up as knowledge and techniques which are applied to the management of buildings. This definition is far less grandiose but makes the same basic point as Duffy: buildings need people and systems to run them. The argument presented by Dorian et al. however, implies that FM is merely a mundane, yet necessary, aspect of an organization whereas Duffy advocates for FM as an integral part of an organization.

The Dorian et al. argument, which was presented by and for FM academics and professionals could, in fact, be more self-serving than it seems. If the management of facilities was simply functional support rather than a driver to fulfillment of an organization's goals, instead of creating an FM department within an organization, everything could be outsourced to one or more facilities management firms. "Total facilities management" is a term meant to encourage organizations to leave all "non-core business" to these FM firms. This term was presented by FM academic David Howard (2002), whose snarky analysis warns of the potential consequences in outsourcing too much of FM to other organizations. He seems to argue that leaving these responsibilities to other firms may be helpful to the bottom line but has implications in the long-term quality of the services.

It is important to discuss the above differences because they point to the confusion among the public to what exactly FM is. Dorian et al. (2012) finds that the public commonly associates facilities management with outsourcing and that "companies engaged in outsourcing are required to offer such a range of disparate services that most fail to do anything well" (p. 258). For many organizations, such as a Montessori school, outsourcing management of intimate building spaces is not within their values and flies in the face of the community that they aim to cultivate. In addition, smaller organizations do not have the resources to hire a team of FM professionals capable of managing such a complex system. How smaller organizations tackle FM is described in the next section. Although the term "facilities management" is broad, and internal differences in its definition have created confusion among the public, it would not be utilized if it did not add value to an organization. 


\subsubsection{FM in Schools and the Montessori Perspective}

Much of the literature surrounding FM spends more time lamenting about its supposed identity crisis than it does attempting to address public confusion around exactly what FM is (see Drion et al., 2012; Duffy, 2000; Howard, 2002). So, what is facilities management? And how is it utilized in schools? The fact that facilities managers have a wide range of skills and that each organization takes a different approach to facilities management means that FM is not the same across organizations. The roles of a facilities manager range from planning how space is used such as inventory management to more day-to-day tasks such as catering, cleaning, and security (Tay \& Ooi, 2001). We can, however, provide concrete examples of how FM is implemented and the systems that enable it, which is discussed in subsequent sections. This section is focused on how FM is implemented in schools, where FM's contributions to the bottom line are less apparent.

Prior to and during the industrial revolution, schools were built to be utilitarian and reflected the philosophy that the setting of education was of little consequence. One-room schoolhouses are widely recognized in American imagery as a pillar of westward expansion and were largely not subjected to governmental oversight. Movement from one-room schoolhouses to vast institutionalized campuses took place over subsequent decades and was motivated by migration to large cities brought on by the growth of manufacturing jobs. Efficiency became paramount to the operation of schools, inspired by bureaucratic theory in manufacturing companies (Kowalski, 2002).

Larger, institutionalized schools brought about conversations into how schools should be constructed and the influence of the "built environment" on student success. Curriculum in school administration graduate programs now included emphasis in FM on the basis that quality of school facilities contributes to learning outcomes (Kowalski, 2002). Indeed, FM has been shown quantitatively to be an outstanding factor in the success of students. In one study, poor management of facilities in several key areas correlated significantly to the delivery of instruction (Duyar, 2010). Analysis by Milwaukee's public school system found that "cosmetic condition" of facilities "may impact student performance more than many social and economic variables." (L. Morgan, 2001, p. 11). Bauregard and Ayer (2018), however, did not find a significant positive correlation between facilities condition and student performance, but suggest opportunities for overall performance improvement to facilities management in schools. All these studies relied on questionnaires from either educators or students. Of course, other environmental factors such as poverty have a role in not only overall student performance, but also in the ability to maintain school facilities. The Duyar (2010) study presents these other factors and notes that much more study is needed to determine causation in these instances. What can be ascertained, however, is that students, staff, and teachers rank the quality of their facilities as an important factor to delivery of education.

As described in section 2.1.1, in a business context, the value of FM is usually determined by its effects on the bottom line of an organization. Although some say the value is more inherent, "operational expenses", like FM, are defined by their return on investment. If the organization is civic or non-profit, as in the case of most school systems, the perceived value of FM can become vague (Beauregard \& Ayer, 2018). In that sense, the argument for a school to allocate significant resources to FM often lands poorly. What studies such as Duyar (2010) highlight is the value that proper facilities management can bring. These studies identified that students and teachers alike 
understand that. However, non-profit institutions are also limited by economic constraints, and allocations for FM are discretionary (Beauregard \& Ayer, 2018). Therefore, given the level of satisfaction FM provides, further emphasis should be placed on resources for FM in the public school system.

Many of the problems with facilities quality described above are the result of specific funding constraints creating an inability for schools to make large improvements. Some problems however, are the result of ongoing neglect to perform simple and low-cost maintenance (Kowalski, 2002). Gradual decay can be difficult to notice on an institutional level without a systematic approach to its prevention through the application of FM, leading to significant costs in the future. Therefore, given that primary and secondary schools have limited resources, a system for managing FM provides necessary tools even for those with limited FM experience, and without needing dedicated staff.

FM has grown throughout the years from paper drawings to fully digitized and intelligently maintained databases (Bröchner et al., 2019). GIS is one tool that has been utilized in the past to enable FM on a school campus. In a GIS, facilities features can be represented spatially, and contain complex relationships. With building data represented "inside" a GIS, these fundamental data can be expanded to provide solutions for facilities management, indoor navigation, emergency response, and strategic planning. The link from the non-spatial to the spatial highlights the power of GIS in providing a better, more understandable model for internal use by an educational institution. It also provides the type of flexibility necessary for a changing classroom, especially when applied to more innovative education systems.

The Montessori method of education gained prominence in the United States during the mid- $20^{\text {th }}$ century as an alternative to the more structured education method found in the American public education system. Its creator, Maria Montessori, recognized the natural inquiry for knowledge that occurs in children and allows them to choose, with guidance, the activities that most interest them. Indeed, the classrooms of a Montessori school should be set up to encourage learning in a way that is useful to the child (Montessori, 1976). According to Bruner (1999, p. 32), "to create interest in a subject is to render it worth knowing," meaning that knowledge acquired in the classroom should be applicable outside of the current context. Classrooms, then, should also not be cluttered with extra or distracting material, as this can be confusing. A Montessori classroom is designed not to bombard the student with superfluous knowledge, but to provide just enough stimulation to awaken the learning young children naturally crave.

The materials in Montessori classrooms are, therefore, thoughtfully chosen both in terms of what the specific item is and the quantity or variety provided (Montessori, 1976). For example, developing and refining the senses is considered among the fundamental pillars of a Montessori education. Sensory materials, suitable for different age groups, are usually present in a Montessori classroom. It is, then, important for the correct materials to be in the correct classrooms. A FM system could allow for inventory tracking across classrooms and ensure that when materials are shared between classrooms they are returned to their appropriate locations.

\subsection{GIS for Facilities Management}

Systems that enable facilities management have proved useful for large and small organizations to streamline their FM goals and reduce the staffing needed. Large 
organizations have entire departments dedicated to managing their facilities, staffed with people who have the expertise to manage complex datasets and deploy them effectively. Responsibilities of an FM department include operating and maintaining facilities, grounds, and infrastructure, thus providing the resources staff-members need for day-today operations and planning for the future. Smaller organizations often do not have the capacity to justify dedicated FM staff. A FM system designed for such organizations should be both powerful and usable by staff members without significant technical skills.

Section 2.1 discussed many of the historical and theoretical underpinnings of facilities management and why organizations require FM. This section discusses the use of GIS as a tool for enabling FM and underscores the power of a GIS as opposed to a more traditional system. Or no system. GIS has the functionality necessary to create a user-friendly tool for managing facilities, but first necessary steps must be taken to ensure these facilities and their attributes are represented accurately.

\subsubsection{Analog to Digital}

For organizations with older facilities, or indeed newer facilities, building information may not exist digitally, and are only accessible in the form of paper floorplans. To create a GIS for FM, these floorplans must be digitized either directly as features in a GIS, or in Computer-aided Design (CAD) files, which are converted to GIS features. The main distinction between CAD and GIS is the presence of features' defined relationships to the world in which they physically exist. In addition, CAD files exist in a local coordinate system, which does not include geospatial data, such as a geographic coordinate system and non-spatial attributes. CAD data are important to the construction and planning process and are useful as pictures, but not as a spatial model containing complex relationships between features. A fundamental aspect of a GIS is that its features have relationships. The features know about each other, which allows for spatial analysis and querying to take place (Wittreich, 1997).

However, what if digital information, CAD or otherwise, is not available for any of the facilities included? There are a few solutions in this case. First is to recreate the entire building from scratch using Light Detection and Ranging (LiDAR) and distance measuring technologies. Another method is to rely on volunteered geographic information (VGI) data where building owners and staff members contribute their own information about the building with user-friendly tools. On a large scale, this method is employed with relative success in projects such as OpenStreetMap. Quality, however, can not be guaranteed with VGI, especially indoors, and the tools used are still in their infancy (Wu et al., 2021).

The last solution, while tedious, is to manually digitize floorplans directly within GIS software such as ArcGIS Pro. Automatic options such as Esri's ArcScan tool, which converts scanned images into vector-based feature layers exist. However, due to the diversity of styles and quality present in floorplans, one would spend more time correcting inaccuracies than they would manually digitizing (Esri, n.d.-g; Wu et al., 2021). Consequently, considering the current state of automatic digitizing solutions, manual digitization is still usually the best option, especially when dealing with older buildings. The process of manual digitization begins with georeferencing, which is discussed in section 2.2.2. During digitization, features are classified to adhere to a chosen data model. This is discussed in section 2.2.3. 


\subsubsection{Georeferencing Scanned Floorplans}

When a scanned image, such as a floorplan, is brought into a GIS, it is known as a raster, and stores values in a matrix of cells. Initially, these rasters do not have geographic information stored with them, meaning that the software cannot determine where they are on the planet. Georeferencing matches specific points on a raster with their known location on the earth, usually against a reference layer such as satellite imagery. A paper floorplan can be understood to be accurate within its own "local" coordinate system. Georeferencing can relate these floorplans to their location on the earth and can then be used in conjunction with data from many sources in a "global" coordinate system (Esri, n.d.-d).

The georeferencing tools in ArcGIS Pro will automatically prompt the user to define a coordinate system and automatically selects the current coordinate system of the map. Georeferencing uses ground control points (GCPs) to cross-reference a point on an internal coordinate system to the chosen geographic coordinate system, thereby assigning that point to a specific location on the earth's surface. When georeferencing, the four fundamental transformations performed are translation, rotation, scale, and skew. This requires at least three GCPs: one each for translation, rotation and scale, and skew (Bolstad, 2019). When georeferencing floorplans, it is important to maintain the aspect ratio of the raster floorplans. Skew, therefore must be avoided and a maximum of two GCPs can be chosen to perform the transformation: one point to move the dataset to the correct location (translation) and another to scale and rotate (Esri, n.d.-a). The two GCPs chosen must be as accurate as possible, and can rely on base data such as imagery or LiDAR. They can also come from point data gathered from highly accurate Global Positioning Systems (GPS) (Bolstad, 2019).

\subsubsection{Digitization and Data Models}

With floorplans georeferenced as accurately as possible, digitization can commence. It is best practice to choose a data model and create all relevant feature classes prior to beginning digitization. This way, the analyst can be aware of how the digitization should take place and it can be easily categorized. Feature classes, in which the digitized features will be stored, contain specific attributes with necessary information for maintaining the integrity of the data. This includes items such as the height of a feature relative to the surface, the building or level where a feature is located, and how that feature is used.

There are at least two widely used data models for representing indoor features with a GIS. The first is the IndoorGML model. This open data model uses simple polygons to represent the room geometry, known as cells. Each cell is then given a centroid point from which connections between cells are identified using lines. In this case, connections usually mean there is a way to pass directly from one cell to the next. The connection lines must contain attributes for both point identifiers to enable the integration of separate connectivity layers. Another building layer can also serve to group cells together (Figure 2-1). The IndoorGML standard also supports the conversion between coordinate systems and from local to global coordinate systems (Tekavec \& Lisec, 2020). 


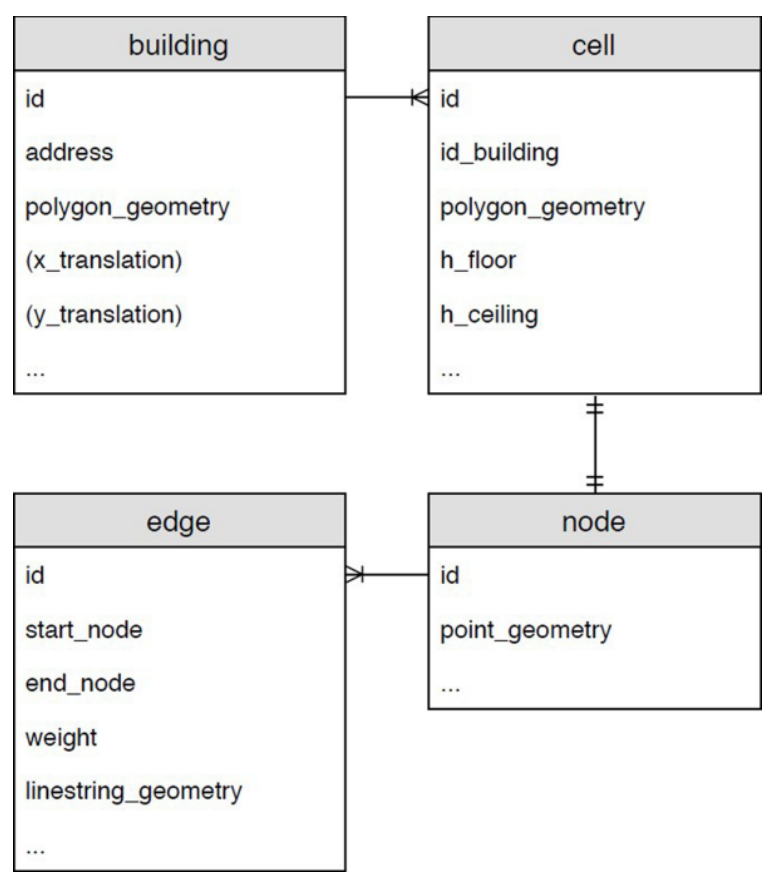

Figure 2-1: IndoorGML Data model (from Tekavec \& Lisec, 2020)

The next model is the proprietary alternative provided by ESRI, ArcGIS Indoors. As is the case with many ESRI products, the structure of the data is freely available, but taking advantage of its more advanced features requires additional licensing (Esri, n.d.b). This data model is meant to enable features like 3 -d representation of buildings, indoor navigation, and interactive displays. The building information is stored in a geodatabase and sorted into six feature classes with a hierarchy representing each level of detail, listed here in ascending order (Esri, n.d.-b):

- Sites - The boundary of the managed site for visualization; Contains all other features

- Facilities - The footprint of each facility

- Levels - The footprint of each level; Contained in a facilities feature

- Sections - Optional feature class representing non-overlapping areas within a level, such as departments or wings

- Units - Individual areas contained by a section or level, such as rooms, hallways, or stairwells

- Details - The lines representing the floorplan such as walls, doors, windows, and stairs

The first five feature classes, representing certain areas in a building, are polygon features, which ArcGIS Indoors uses to assume hierarchy in the data and relate attributes correctly. The "Details" feature class stores line data that represents the floorplan. When digitizing from scanned floorplans, new line segments are created to match the lines on the floorplans. The lengths of segments can either be derived from other features or from the dimensions denoted in the floorplan. It is usually best practice to draw lines by manually inputting their lengths where available (Tekavec \& Lisec, 2020). The final aspect of an ArcGIS Indoors database is the point data. That is, the features within units that are to be represented. In ArcGIS Indoors terminology, these are called 
PointsOfInterest. These features might represent any doorways, stairwells, assets, or classroom materials and can be customized based on the desired features of the map (de Jong et al., 2021).

\subsubsection{Web GIS}

For a GIS to be accessible to a wide range of people and abilities, it must be user-friendly and available on a wide range of devices. Traditionally, the model for a GIS assumes that the system will only be useable with specialized software and require immense expertise to create and maintain it. For organizations looking to implement GIS, this often proves a barrier-to-entry. Smaller organizations, such as schools, do not have the resources to hire dedicated staff to run their GIS. Moreover, the traditional model of GIS is not suitable to the use-cases of modern GIS projects. Today's projects can utilize many contributors, each requiring the most up-to-date data. Users require immediate access to data, from any device. With web GIS, analysts and users are not confined to one machine, nor must they run specialized software to access their system.

Web GIS takes advantage of modern cloud computing technologies to deliver content with minimal computing power on the user's end. The web browser on the user's device serves as the client, with a webserver responding to the request. There are two main approaches to client-server architecture: thin client and thick client. In thin client, the majority of the processing is done on the server side. The response from the server is generated and presented to the client in a web-friendly format. For spatial web applications, the GIS server sits behind the webserver and can be called only through the webserver. In other words, the GIS server delivers the data to the webserver, which delivers it to the client. This is advantageous in cases where a GIS system requires considerable flexibility, and the ability to run on relatively low-end hardware, such as Google Chromebooks and iPads. Tradeoffs of the thin-client method are limited to the functionality and capacity of the server (Agrawal \& Gupta, 2017). The thick-client method enables more computing to take place on the client side through browser plugins or applets, which are locally running applications meant to support online content. Vector rendering, for example, could be done by the client device, meaning the data can be delivered in their raw form. Of course, this means that the hardware and software capabilities on the client side must be considered (Agrawal \& Gupta, 2017).

Over the last decade, significant improvement has been made to the capability of web GIS. For example, ArcGIS Indoors (section 2.2.3) is a relatively new product from ESRI and combines the aforementioned server-based content delivery with newer capabilities of modern devices. This enables further interactive features such as indoor navigation by harnessing Bluetooth Low Energy (BLE) and Wi-fi fingerprinting (de Jong et al., 2021). In this case, the server is utilizing information provided by the device to deliver interactive content. In addition, servers themselves have become more powerful, more reliable, and cheaper. It is no longer normal for organizations to run their own servers to deliver content, save for the largest organizations. Server resources are largely commodified and are thus treated as a utility. Most major tech companies offer their own cloud computing product (Agrawal \& Gupta, 2017). Microsoft Azure, Amazon AWS, and Google Cloud, for example, accounted for $58 \%$ of total "cloud spend" in Q1 of 2021 (Stalcup, 2021). In other words, the responsibility of maintaining complex server infrastructure is not the responsibility of the organization. 
In an educational setting, the advantages of web GIS are more apparent than ever. Baker (2015), who works for Esri on its educational products, cites a few reasons why desktop GIS was never widely adopted in K-12 education, including limitations in IT support, data storage, hardware, and supportive curriculum. With web GIS, interfaces can be tailored to the needs of the user and account for age level and instructional objectives. Collaboration can also take place, allowing multiple users access to the same maps from their own devices. Further, institutions can take advantage of products such as Esri's ArcGIS Online, which hosts content on servers provided by Esri, eliminating the burden placed on, or even the need for, IT support staff (Baker, 2015).

\subsection{Applications of GIS for FM}

GIS has taken hold as a preferred solution to the facilities management problem. All information can be stored spatially and assets can be viewed and managed in the context of the environments they support. For example, air conditioning (AC) units have a relatively predictable life cycle. In a GIS-based FM system, all the AC units of a building could be visualized together, immediately indicating which need replacement. Events, such as maintenance requests, can also be pinpointed to their exact location and assigned priority or status, providing a better understanding of the specific issue.

\subsubsection{Digitizing and Map Creation}

A high-quality basemap that includes interior features is a fundamental component of a FM GIS. This includes both interior and exterior features. Interior features can be gathered from both public and private sources in a variety of formats, including scanned paper drawings, CAD files, and the more advanced Building Information Modeling (BIM). Bahri et al. (2019) created a campus basemap for Universiti Kebangsaan Malaysia by scanning and manually digitizing paper floorplans directly inside the GIS. The floorplan locations were verified using remotely sensed and GPS collected information to place the building footprints. This process was considered by the authors to be an important step as it ensured optimal accuracy. They also used previously created CAD data where available (figure 2-2). Similarly, the Jomo Kenyatta University of Agriculture and Technology created a campus basemap through the digitization of paper floorplans. This solution used a line layer to trace the floorplans segment-by-segment. The floorplans were first digitized into AutoCAD .dwg files, then imported into a geodatabase and georeferenced (Mwaniki \& Odera, 2014). 


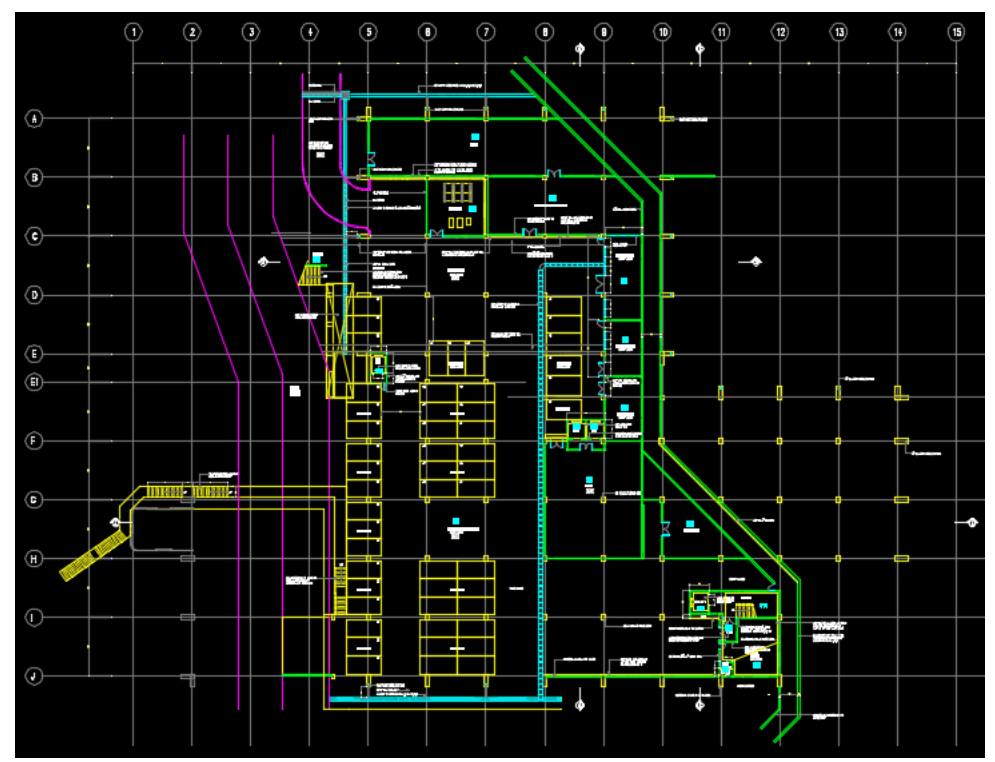

Figure 2-2: Example of a building floorplan (from Bahri et al., 2019)

Tekavec and Lisec (2020), in developing a solution for indoor modeling, also created GIS-based floorplans through digitization, but in a simpler manner. Rooms were digitized by drawing polygons, instead of individual segments, eliminating the need to later create more complex features. Morgan (2009) brought the FM platform for the University of South Carolina from a fragmented CAD floorplan model to an integrated GIS model by georeferencing CAD floorplans. Using two ground control points collected with GPS, the CAD floorplans were accurately located in the GIS. In this case, all buildings were already available in CAD files and no digitization was required. Bingaith (2010) also completed a major individual project (MIP) for the University of Redlands MSGIS program in which CAD files were georeferenced and brought into a GIS for the California Institute of Technology.

Other solutions have employed automated techniques to varying success. Wu et al. (2021) developed an approach that detects building walls to a high-degree of accuracy, although requires input data of very high-quality and is subject to design choices of the floorplans themselves. Wu's approach also can only handle rectangular walls, not curved walls. Dodge et al. (2017) tested several image-parsing methods on floorplans and found generally poor results owing to the vast style differences among different floorplans (Figure 2-3). For an image-parsing system to work reliably, it would have to be uniquely set up for each floorplan. They see these methods as useful for extracting certain information such as room size, but not in total recreation of floorplans
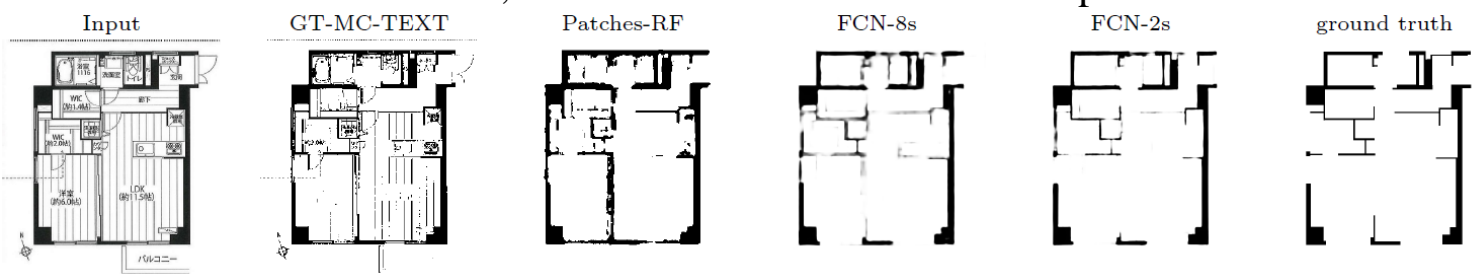

Figure 2-3: Various image-parsing methods (adapted from Dodge et al., 2017) 


\subsubsection{Deploying FM}

Applications of GIS for FM are wide-ranging. The Kuwaiti Ministry of Education developed a GIS-based FM application, which integrates BIM models of selected schools in Kuwait with tools for space and asset management, emergency planning, and other areas of FM. Their goal was to create a solution that is useable by the entire Kuwaiti school system, and is accessible with a desktop software application. The base application provides users with room and utilities information and allows for rooms to be reassigned and adapted for future use. It also stores maintenance data, including scheduled maintenance and replacement as well as requests from staff and students (ElGamily \& Al-Rasheed, 2015).

Bhari et al. (2019) created a system that integrates data from multiple sources as existing maintenance data was stored separately from spatial data. After integrating spatial information into a central geodatabase, they established database connections to the other facilities data, joining through standardized room numbers. The system is hosted on University servers and utilizes an ArcGIS Enterprise Dashboard to serve as the front-end user interface (UI). Unlike the El-Gamily \& Al-Rasheed project, this system is managed entirely on the server side and can be accessed through a web browser. The Morgan (2009) project also required a desktop application to operate and only stored attribute information for individual rooms. It did not provide many interactive abilities like maintenance requests or the ability to easily update attributes.

\subsection{Summary}

The academic debate surrounding the function of FM is a product of differing philosophy in the role FM is meant to have in an organization. Some view FM as fundamentally important to an organization, and as such should be integrated and indispensable (see Duffy, 2000; Tay \& Ooi, 2001). Others see FM as best outsourced to firms that specialize in certain facilities operations (see Drion et al., 2012; Howard, 2002). Both takes are selfserving to the field as the former supports hiring dedicated personnel within an organization while the latter advocates for the growing industry of specialized FM firms. In an American educational context, the concept of the built environment's influence on student learning came about through the onset of the United States' institutionalized and centralized public school system (Kowalski, 2002). Several studies (see Beauregard \& Ayer, 2018; Duyar, 2010; L. Morgan, 2001) show correlation between student success and properly managed facilities, but also cite other factors as contributors and call for more research into the subject. FM is important in the context of a Montessori school as the materials present are thoughtfully chosen and reflect the values of the Montessori system.

From a GIS perspective, the technology has been widely utilized to create FM solutions represented spatially. The basic procedure to creating a GIS system for FM is to (a) establish a consistent indoors data model, (b) represent building floorplans on a global coordinate system by either georeferencing CAD files or digitizing paper floorplans, (c) assign attributes used for FM functions, and (d) publish the data in an accessible format. Web GIS is a popular solution for running the system as it provides user-friendly features that are accessible from many devices. Projects deploying GIS for FM and other indoor mapping applications are widely documented (see Bahri et al., 2019; Bingaith, 2010; El- 
Gamily \& Al-Rasheed, 2015; Mwaniki \& Odera, 2014; Tekavec \& Lisec, 2020; Wu et al., 2021). This project will build on the techniques adopted by previous projects and utilize recent advances in GIS methods to build a system that is useable by people without substantial GIS skills. 


\section{Chapter 3 - Systems Analysis and Design}

The Montessori in Redlands School (MIR) wanted to create a cohesive facilities management system that would give staff members a centralized solution for managing classroom inventory and long-term asset maintenance. They also wanted to provide first responders access to a high-quality map of the campus, including building interiors. The campus basemap created to satisfy these requirements would also provide the foundation for other implementations of GIS in the future. Initial meetings with the client helped to identify these problems and solidify the requirements.

\subsection{Problem Statement}

For several years, the facilities staff at MIR had acknowledged a need for digitized building interiors to provide facilities management solutions and other necessary information about the campus. For this project, MIR staff proposed three high-priority problems that would take advantage of the basemap. These problems were among many others identified; the ones chosen were the most urgently needed and reflect many of the realities facing schools today.

The first problem faced by the school is managing of classroom inventory. Currently, inventory is managed using pen and paper, making the information fragmented or not available at all. Another problem was identifying the location of assets for longterm maintenance and replacement. The school had a reserve study conducted in 2008 to assist with the long-term planning of permanent school assets such as HVAC systems and water heaters. This information currently exists in paper-form or in spreadsheets and wasn't managed centrally, which presented communication issues among staff members responsible for these assets. Also important was the school's emergency response preparedness. First responders did not have access to important location information that would assist in an emergency, such as door numbers. This information is critical in quickly identifying the location of the emergency and planning effective escape routes.

\subsection{Requirements Analysis}

The requirements verification and analysis portion of this project included a set of preliminary meetings with the client that identified all relevant information and ensured all the materials and data are available (and in what form). This established the client's expectations for the project and verified the scope. The requirements also established the client's role in project completion. A project plan was provided to the client for review and approval.

\subsubsection{Functional Requirements}

The functional requirements define what information is inputted into the system, what the system does with that information, and what results it outputs. A geodatabase was required to hold all the digitized building data and the facilities management (FM) data. Data from this geodatabase were then published to ArcGIS Online in the form of three Web Maps: one each for inventory management, asset management, and emergency first 
responders. The system can run entirely in ArcGIS Online and data are viewable through both a web browser and mobile app and editable in a web browser. Functional requirements are outlined in table 2 .

Table 2. Functional Requirements

\begin{tabular}{|l|l|}
\hline Deliverable & Functional Requirement \\
\hline Scanned floorplan files & $\begin{array}{l}\text { Scanned in high-dpi (Dots Per Inch) resolution } \\
\text { Pixels are encoded in 1-bit black and white }\end{array}$ \\
\hline Campus Basemap & $\begin{array}{l}\text { Uses ArcGIS Indoors data model } \\
\text { Accessible to the FM system } \\
\text { Represented as line and polygon data }\end{array}$ \\
\hline attributes & $\begin{array}{l}\text { Includes features for classroom materials inventory, asset } \\
\text { maintenance and replacement, and door numbers } \\
\text { Accessible to the FM system } \\
\text { Represented as point data }\end{array}$ \\
\hline ArcGIS Online System & $\begin{array}{l}\text { Uses data deployed to ArcGIS Online } \\
\text { FM features and attributes editable in the web interface } \\
\text { Includes FM features } \\
\text { Is floor-aware }\end{array}$ \\
\hline Field Maps Deployment & $\begin{array}{l}\text { Derived from Web Maps } \\
\text { View campus features in FieldMaps mobile app } \\
\text { Add and edit FM features in FieldMaps mobile app } \\
\text { Is floor aware }\end{array}$ \\
\hline
\end{tabular}

The floorplans were to be scanned using an optical scanning device that can accommodate the large paper size and can sense at an adequate resolution. In the resulting raster image, the pixels were required to be encoded in 1-bit color depth as either black or white. This encoding option was chosen to help reduce file sizes. It also produces shapes with effectively infinite contrast, which optimizes the images for optical character recognition (OCR) and other automated scanning software. The campus basemap and FM features were required to use the ArcGIS Indoors data model (described in section 2.2.3) which allowed for the system to recognize buildings with multiple floors and correctly display features. Also to adhere to the Indoors model, the campus basemap was stored as line and polygon data while the FM features were stored as point data. 
Functional requirements for the Web Maps were that they should be deployed separately for each intended use. The campus basemap was present in each Web Map and included FM features based on purpose. They were also floor aware, taking advantage of the Indoors data present in the attributes of the layers. Further, the FM features and attributes are editable in the Web Map or in a series of Web Apps. The Field Maps deployment has similar requirements, although what it displays was derived from the Web Maps.

\subsubsection{Non-functional Requirements}

Non-functional requirements define the expectations of the client in the look and feel of the deliverables. A common theme for these requirements was useability, which is essential to the success of the deployment. Since the system was designed to be used by people who are not GIS professionals, it was required to be easy to use and accessible without running ArcGIS Pro or other desktop software. The system also used a consistent design with symbology that clearly indicates the function of each of the features. Further, it was important that the system be reliable and easily accessible using licensing already owned by MIR. Non-functional requirements are defined in table 3.

\section{Table 3. Non-functional requirements}

\begin{tabular}{|c|c|}
\hline Deliverable & Non-functional Requirement \\
\hline Scanned floorplan flies & Stored in standard tif file format \\
\hline Campus Basemap & $\begin{array}{l}\text { Consistent and clear symbology } \\
\text { Accurate to scanned floorplans } \\
\text { Includes indoor and outdoor features }\end{array}$ \\
\hline $\begin{array}{l}\text { FM features and } \\
\text { attributes }\end{array}$ & $\begin{array}{l}\text { Consistent and clear symbology } \\
\text { Attributes are useful to type of feature }\end{array}$ \\
\hline Web Maps & $\begin{array}{l}\text { Always available } \\
\text { Stable } \\
\text { User friendly interface } \\
\text { Hosted by ArcGIS Online }\end{array}$ \\
\hline Field Maps Deployment & $\begin{array}{l}\text { Always available } \\
\text { User friendly interface } \\
\text { Hosted by ArcGIS Online }\end{array}$ \\
\hline
\end{tabular}




\subsection{System Design}

The system design includes the online storage of the campus basemap and FM features, which were derived from a file geodatabase produced in ArcGIS Pro. The file geodatabase contains a feature dataset that includes feature classes for the campus basemap and separate feature classes for the FM features. The file geodatabase was published to ArcGIS online as hosted feature layers. Three Web Maps were created for each of the three use-cases for the system, which are deployed to ArcGIS Web Apps to enable the floor-aware capabilities and editing features of the system. Each of these maps are also viewable in the Field Maps mobile application. The online system only has the capability to edit the FM features, as the campus basemap relies on features only present in ArcGIS Pro. If changes need to be made to the campus basemap, they will have to be republished as feature layers.

The schema for the campus basemap followed the ArcGIS Indoors data model. The FM features also included data to enable indoor operability. Other attributes for the FM features were agreed to prior to the project commencing. The editing tools take advantage of the editing widget for ArcGIS WebApps. Figure 3-1 outlines the system, including data creation and the functionality of the system itself.

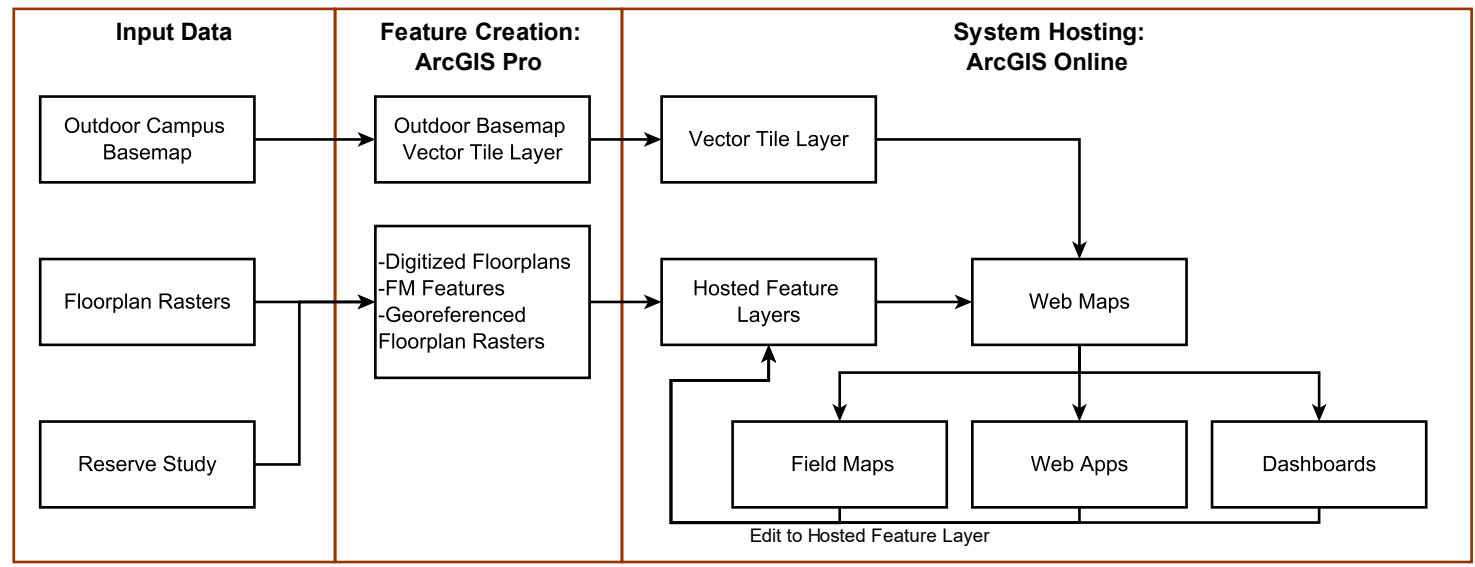

Figure 3-1: System Architecture

\subsubsection{Data Creation and Editing with ArcGIS Pro}

Input data were provided by the client and include the paper floorplans, a previouslycreated outdoor campus basemap, and a reserve study. The reserve study serves as the basis for the asset maintenance and replacement features. Sources of the input data are discussed further in section 4.3. ArcGIS Pro was utilized to georeference the floorplans, digitize them, and apply the ArcGIS Indoors data model. Ground control points (GCPs) for georeferencing were placed using a combination of aerial imagery and LiDAR layers for the Redlands area. To avoid distortion, two GCPs were selected for each raster image. Digitizing was conducted using the editing features in ArcGIS Pro. To ensure the digitizing process was accurate, where possible, lines were drawn by inputting the dimensions denoted by the floorplans. The editing grid was utilized to ensure lines were correctly offset from each other and met at correct angles. Polygon features were drawn according to the outline created by the line features. Topology checks were also applied 
to both the line and polygon layers to avoid slivering and dangles which, respectively, are instances where polygons do not perfectly coincide and lines whose nodes are not snapped to another line.

Initial FM features were created as point data in ArcGIS Pro and are stored in their respective feature classes. Attributes for each point are inputted as the feature is created. Further points can be added and existing points can be edited in the online system through either the Web Apps or the Field Maps mobile application. It is important at this stage in the feature creation process to determine the room in which the point is located and its approximate location. Because this system is being deployed without ArcGIS Indoors licensing, that information cannot be determined automatically. These layers were then uploaded to ArcGIS online as hosted feature layers, which serve as the basis for the rest of the system components. While all the FM features can be created and edited online, if one wishes, they could also open and edit the FM layers in ArcGIS Pro.

\subsubsection{The ArcGIS Online System}

ArcGIS Online was selected for its stability, easy UI, and operability with ArcGIS Pro. As explained in the previous section, layers, their data, and their attributes are created in ArcGIS Pro and uploaded to ArcGIS online as Hosted Feature Layers using the ArcGIS Pro "Share as Web Layer" tool. From hosted feature layers, they are published to Web Maps. Design was important in these maps, as how the layers are stored and displayed affected the overall useability and integrity of the map and its features. It was important that the campus basemap, including indoor features was not editable in the system, as online editing tools do not have the aligning, snapping, and grid features present in ArcGIS Pro. If a user of the system wants to alter the basemap they would have to change them using ArcGIS Pro. Note here that campus basemap refers to both the Hosted Feature Layers that store the indoor features and the outdoor map, which is stored as a vector tile layer. A vector tile layer is a layer that stores multiple vector layers and their symbology as one set of features. Two advantages to using vector tile layers for a basemap are that the map legends only contain one outdoor basemap layer, reducing clutter, and the tiles are pre-rendered across a range of scales, improving performance.

The hosted feature layers and the vector tile layer are then used as the basis for the Web Maps. In this system, there are three Web Maps for classroom inventory, asset maintenance, and emergency first responders. These Web Maps are then utilized in a subsequent two Web Apps, two Dashboards, and in the FieldMaps deployment. Web Apps were chosen for the online interface because of their customizability, ease of use, and that they make available both editing widgets and floor-aware widgets. Dashboards are used when information needs to the rapidly displayed, but not edited. The ability to view and edit on the web is one of the most important aspects of this project, as such availability of these features were strongly considered when selecting a product to use.

\subsection{Project Plan}

The project was scheduled to begin on September 2, 2021 and end November 14, 2021. The original schedule was created to coincide with the spring semester of the University of Redlands fall semester schedule. The end date in mid-November is intended to allow enough time for changes that cause the schedule to run long and for writing this report. It 
also represents the agreed-upon date when all aspects of the project that are essential to the client will be completed. There were a few changes.

The main tasks of this project were requirements verification, scanning, floorplan digitization and map creation, creating FM features, publishing to ArcGIS Online, and deploying in FieldMaps. Requirements verification included a preliminary meeting with the client to gather all the data, collect materials, and define the scope. It also established the client's responsibilities throughout the project. Image scanning involved scanning approximately 160 separate drawings and naming them appropriately. The majority of the files were not utilized for the project, but since the older drawings were badly damaged, all were scanned to preserve for future use. Because of the volume of files, scanning took about a day longer than anticipated. The initial project plan is outlined in figure 3-2.

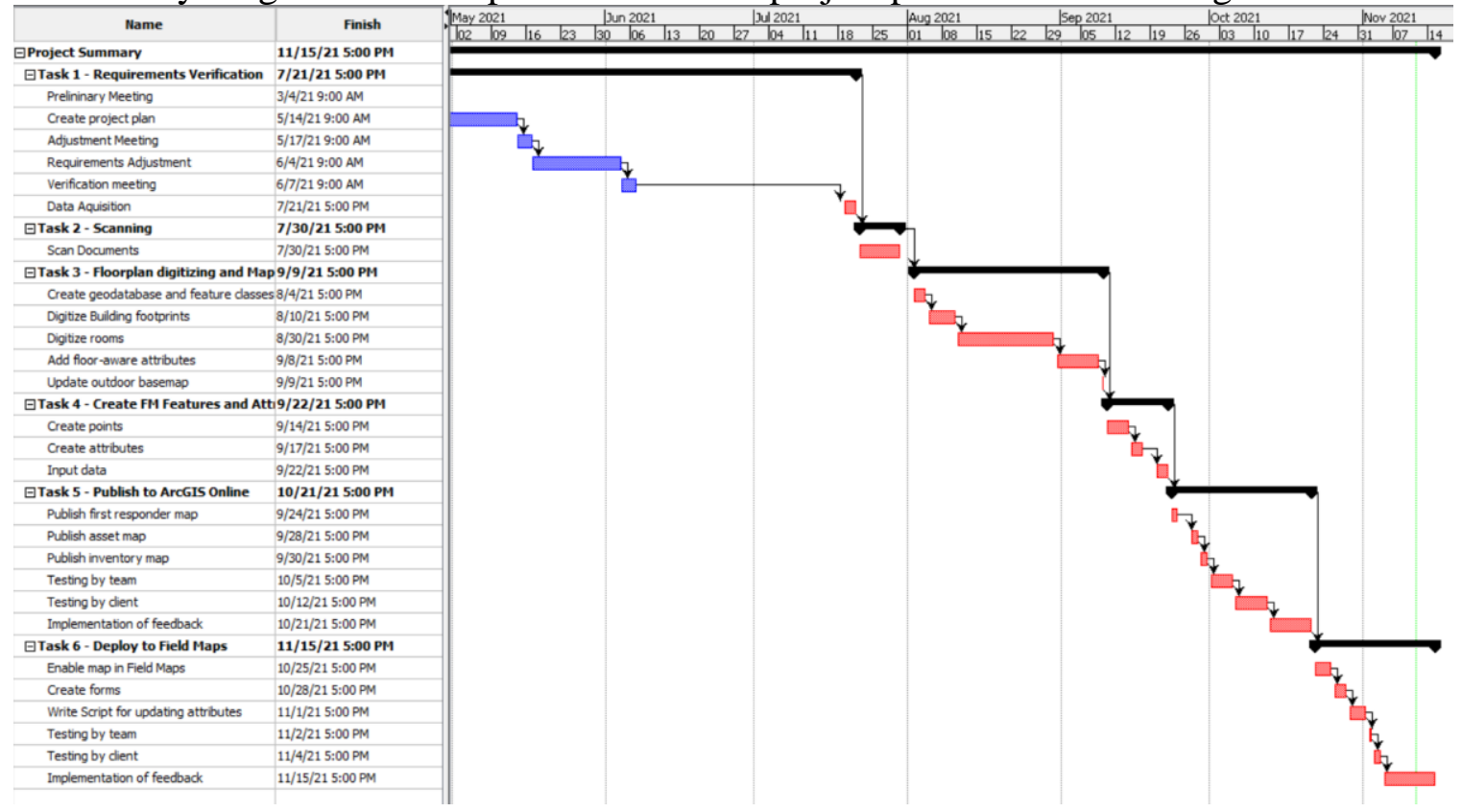

Figure 3-2: Initial Project Plan

Floorplan digitization took place in ArcGIS Pro as did the creation of FM features and attributes. These were then published to ArcGIS Online and deployed to Field Maps. In reality, the initial project plan became outdated during the digitizing phase (Task 3 ), which took far longer than expected due to reconciling differences among floorplans and delays from the volunteer assisting with digitizing. As a result, the waterfall method that was to be utilized was modified to allow the creation of FM features and attributes (Task 4) to run concurrently with Task 3 . The actual completion date of the project was about 10 days behind schedule. The updated project plan is outlined in figure 3-3. 


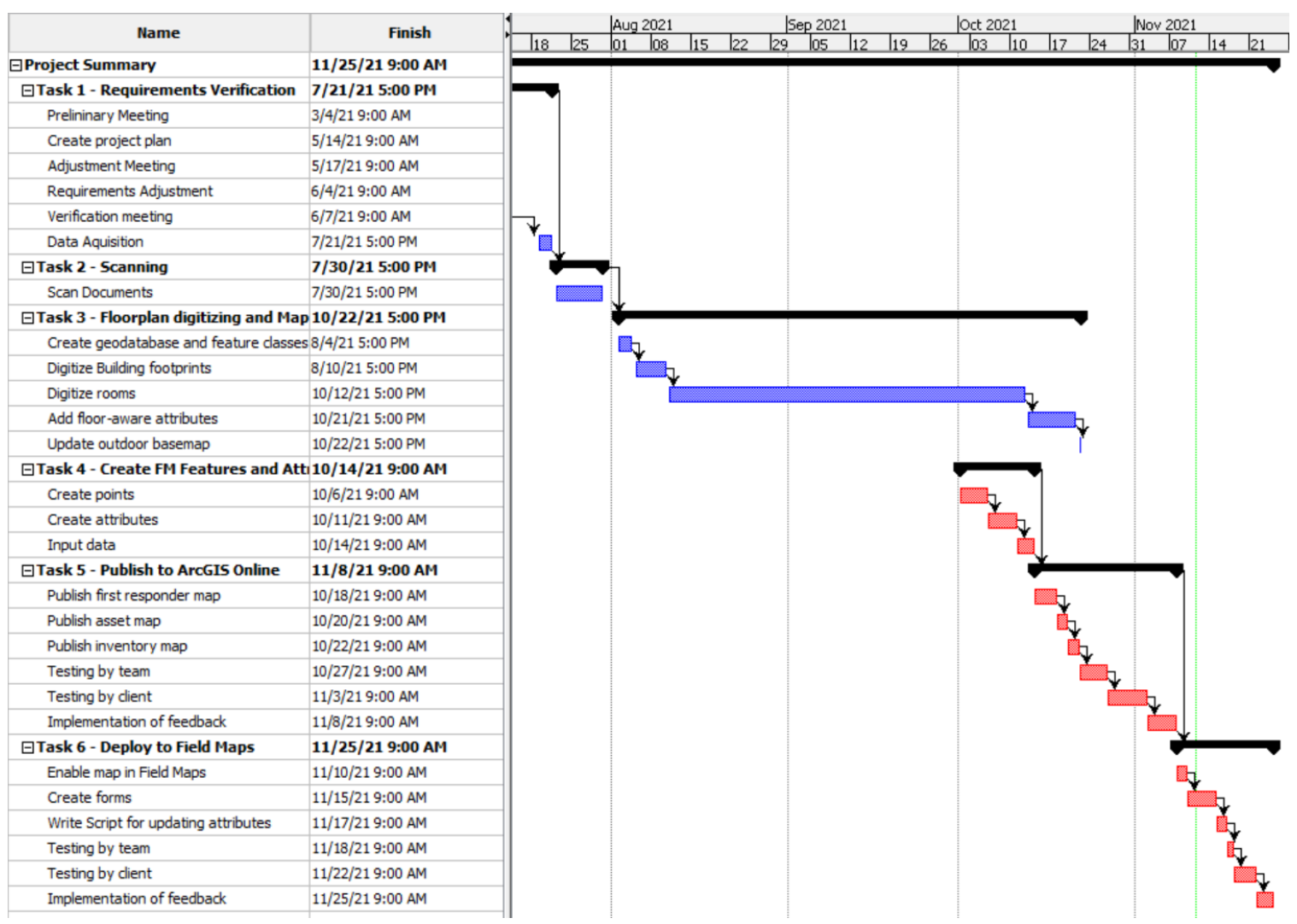

Figure 3-3: Updated Project Plan

\subsection{Summary}

MIR required a FM system that includes features for classroom inventory, asset management, and emergency first responders. This project utilized GIS to create a FM solution at MIR. Functional requirements include scans of very high quality, a campus basemap that adheres to the ArcGIS Indoors information model, and a web interface that can take advantage of the model. The system was to be hosted on ArcGIS Online and remain stable and available at any time. Non-functional requirements ensured the map was easy to understand and use and that the data were accurate.

The system took input data from a variety of sources. Initial features, including the entire campus basemap, were created using ArcGIS Pro. The features were then deployed to hosted feature layers in ArcGIS Online, which were used in Web Maps. These Web Maps were the basis for the Web Apps, which serve as the online interface and the Field Maps deployment. The project was scheduled to begin in September 2021 and end in November 2021, allowing time to respond to changes as they arise. Due to delays in the digitizing process, the deliverables were actually completed about ten days behind schedule. 



\section{Chapter 4 - Database Design}

A well-constructed geodatabase is important to any GIS. The core of this GIS is a spatial representation of the MIR campus, which is accompanied by the FM features and their attributes. This chapter discusses the conceptual design gathered from the requirements outlined by the client, followed by the logical implementation of said requirements. The logical model aims to fit the operations depicted in the conceptual model. However, it also considers technological and practical limitations presented by the software and in the particular use-case of MIR. Also discussed in this chapter are the specific sources of the data and how data were brought into the system.

\subsection{Conceptual Data Model}

As this project aims to provide a solution for managing classroom inventory and assets, the conceptual data model represents how each item is added, removed, or manipulated on a day-to-day basis. The school campus can be divided into either indoor or outdoor area, with property items contained in either area. The property item refers to a material object that is managed by the school and has the attributes of location, type, and quantity. The item type represents whether or not the item is a permanent fixture of the campus or is moveable/consumable. An MIR staff member or other third party interacts with these items by adding, removing, relocating, and performing maintenance.

The property item is a generalization of asset item and inventory item. Asset items are tracked over space and time and replaced at a predictable interval. They are also considered as permanent fixtures of the building. An asset item can be composed of other asset items. For example, the item "skylight" could be a single item composed of many skylights whereas a hot water heater is a standalone item. When the time comes for replacement, asset items are replaced with new asset items. The safety item is a specific type of asset that pertains to the safety of the physical campus and its personnel, such as entryways, fire alarm systems, and evacuation areas. Inventory items are tracked over space and added, removed, or replaced as needed. A bookshelf, for example, can be broken and replaced or moved from one classroom to another. An inventory item can contain other inventory items such as a shelf holding toys. The conceptual data model is outlined in figure 4-1. 


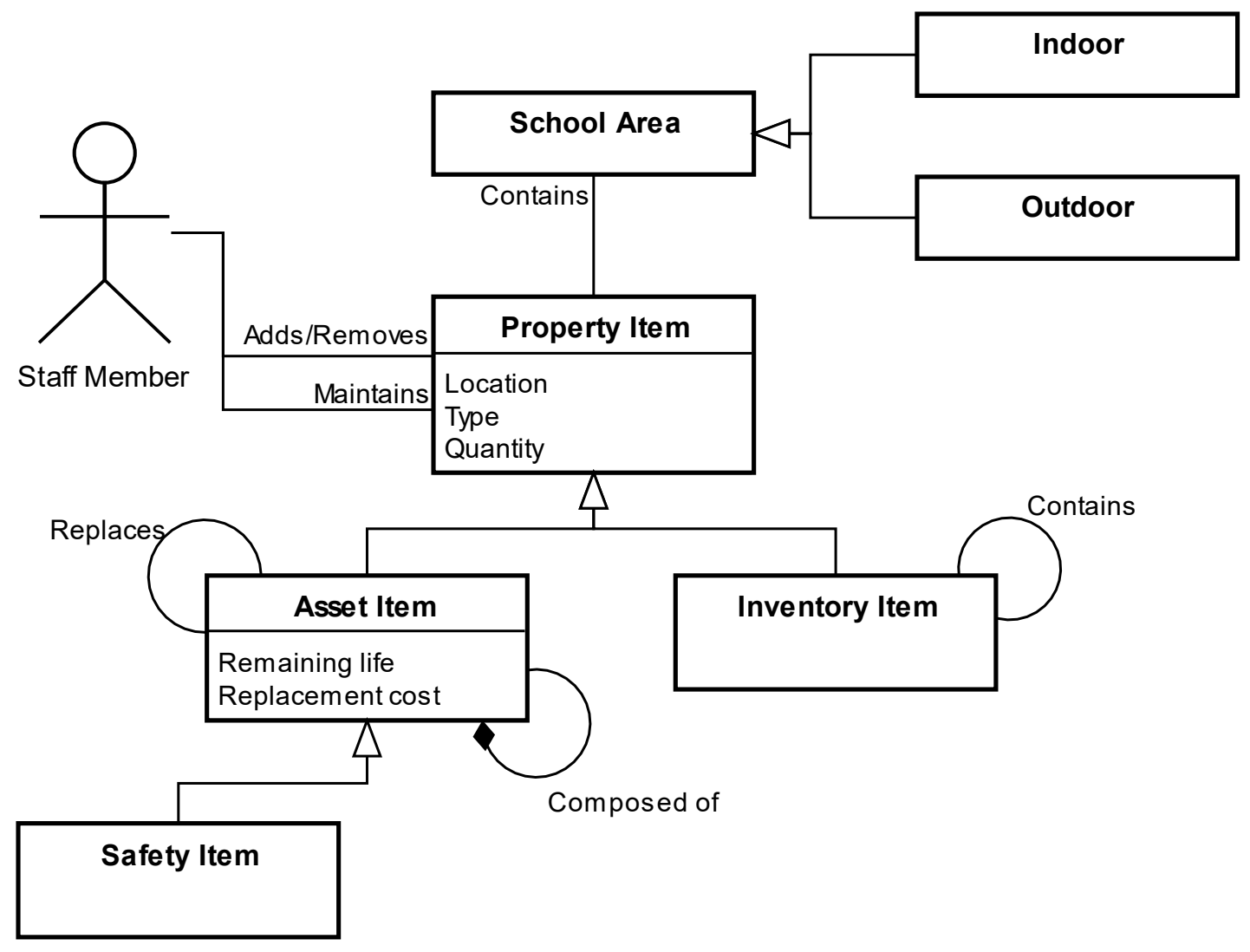

Figure 4-1: Conceptual Data Model

\subsection{Logical Data Model}

The logical data model describes the structure of the geodatabase in which the information is stored. The indoor features use the ArcGIS Indoors data model, which is organized hierarchically based on the granularity of the data (Esri, n.d.-b). The FM points also contain location information from the ArcGIS Indoors data model. In the initial deployment of the system, three feature datasets (FDs) were created: Safety, Inventory, and Assets, each of which contains one feature class. Respectively, these are Doors, Inventory_Locations, and Assets. More point feature classes can be created in the future to support further applications of the system. For example, Safety can be expanded to include evacuation safe zones. Figure 4-2 outlines the logical data model. 


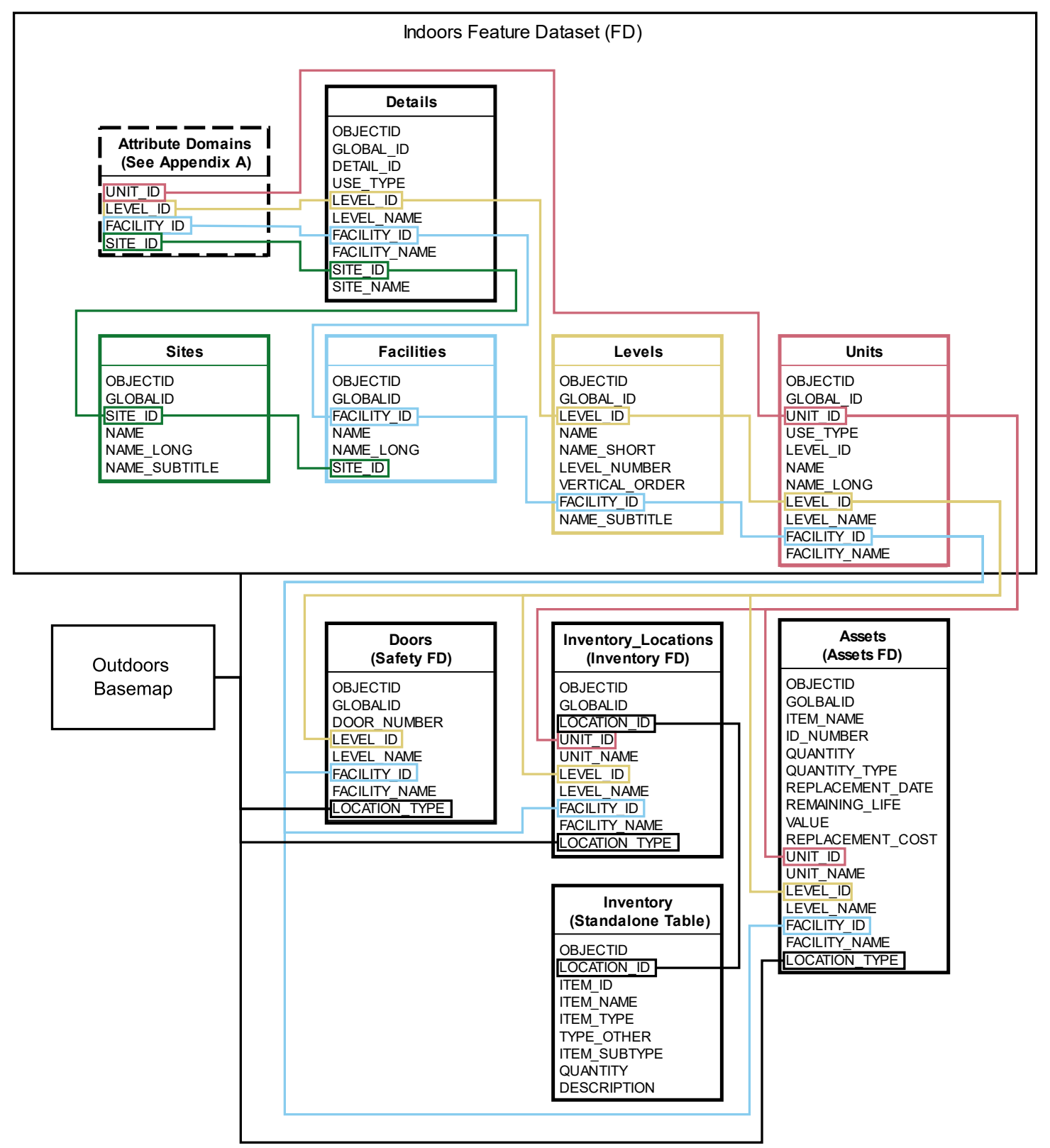

Figure 4-2: Logical Data Model

Indoor features are organized into five feature classes (FCs), each containing relevant location information and are stored in the Indoors FD. The Details FC is a line FC that reflects the interior details of the building floorplan. The four other polygon FCs, which are derived from the Details FC, are Sites, Facilities, Levels, and Units. Sites is one polygon that represents the entire land area of the campus. Currently, there is only one site in the database, but it is set up to accommodate more if necessary. Facilities polygons are the footprints of buildings and Levels are the outline of each floor in the building. Units polygons represent individual rooms or areas. Each of these FCs must fully contain the other. In other words, Units features must be fully contained within Levels, Levels must be contained within Facilities, and Facilities must be fully contained 
within Sites. Information on the capability of the ArcGIS Indoors platform can be found in section 2.2.3.

Each feature has a unique ID associated with it, which is used to identify the individual feature and reference its parent. Units, for example, contains a UNIT_ID field along with a LEVEL_ID and FACILITY_ID field, reflecting the fact that a unit can only be located on one level in one facility. All indoors features must have these fields populated for the system to function correctly. This also includes the FM point features, however, these features can be located either indoors or outdoors. In the case of an outdoor feature, the ID fields can be left null, instead setting the LOCATION_TYPE field to "Outdoors". Each of these fields are populated using attribute domains (Appendix A) to ensure the values are consistent.

The Inventory_Locations FC, contained within the Inventory FD, is the point referencing the location in which items are stored. This FC was a challenge to design as the client wanted to avoid creating lots of points for individual items and wanted the capability for a single point to represent containers of items. For example, a shelf is an item that can hold textbooks. The solution was to create a related table "Inventory" that holds a list of items, each of which reference the point they are located. Inventory items contain attributes for their location: the item name, type, and subtype, the quantity, and a description.

The Assets FC is stored in the Assets FD and stores attributes for the name, ID number, quantity, replacement date, remaining life, value, and replacement cost. Data stored in these attributes are derived from a reserve study conducted in 2008, which contains data through 2037. Assets can be recorded as either one point of a certain quantity or as many points representing multiples of the same item. For example, skylights may be represented as a point for each individual skylight - as opposed to one point for many skylights - to help with locating. Alternatively, they can all be represented by one point in the center of the building if the attributes are the same. The Doors FC, contained within the safety FD, has an attribute for door number, which also serves as a unique identifier. In the conceptual model, asset items are a generalization of safety items. However, the distinction between the two requires them to be stored and displayed separately. In the case of doors, all the doors of a building may be considered one asset and tracked as a whole, but in the interest of safety individual entry/exit points must be tracked separately.

The outdoors basemap is not stored in FCs, but as a tiled vector layer, meaning layers can't be turned on or off and the features do not contain attribute information. This was meant to reduce complexity and increase performance of the system. Therefore, the outdoors layer does not contain any spatially defined areas. As mentioned above, features can be located outdoors, but are not defined as belonging to a certain area.

\subsection{Data Sources}

Input data were sourced entirely from the client, except for the outdoor basemap which was created from satellite data by Phil Sanchez, an Esri employee who is a parent of a MIR student. The floorplan drawings were only available in their original, paper form and were scanned and georeferenced in ArcGIS Pro. The main building has undergone several renovation projects since it was built. Two major projects were reflected in subsequent floorplan drawings, while others were not shown anywhere. The North, 
Grove High School, and Student Services buildings have never undergone major renovations, so their original floorplans remained accurate. Traditionally, two versions of a floorplan exist: the pre-construction drawing and the "as-built" drawing, which shows changes that were made during construction (Ellis, 2021). The buildings on the MIR campus only had pre-construction drawings available. The floorplans are reflected in the indoors feature dataset.

Asset item data came from a 2008 reserve study the school commissioned to plan for upcoming costs. They were delivered as scanned charts containing year-to-year information on the item's value, useful life, remaining life, and the current financial liability. Door locations and their associated number were also delivered in paper form. An initial set of inventory items was provided via an Excel spreadsheet and were entered into the database. Additional inventory items were collected by MIR staff members after the system was deployed. The outdoor map was delivered by Mr. Sanchez in a File Geodatabase.

\subsection{Data Collection Methods}

Data collection followed the initial requirements and planning meetings with the client. Most of the data were provided by the client, including all floorplan and FM data. It was initially planned for the outdoor map to utilize layers from a GIS created for MIR by Wendy Wallace (2010). However, this map proved to be unavailable. A less detailed, but more recent, outdoor map was sourced from Mr. Sanchez. Aerial imagery for georeferencing was obtained using an Esri basemap.

\subsection{Data Scrubbing and Loading}

As described above, the floorplans were delivered in paper from along with other pages included in the original construction plans. The pages were scanned into tif files at 500 dpi resolution using 1-bit black or white color depth and lossless compression, meaning no detail is sacrificed to save storage. In total, 159 pages were scanned, and included the entirety of the building plans. Most of these pages were not used for the project but were included as a deliverable for preservation purposes. Eleven pages were georeferenced across the six buildings, comprising separate levels, spaces, and renovations. Other pages provided valuable information that was cross referenced with the floorplan. Door and window schedule pages, for example, provided necessary dimensions.

The main building presented the greatest challenge during the digitizing process. In its 31-year history, several renovation projects were completed, some of which had accompanying floorplans, some of which did not. The toddler rooms on the west side of the building, for example, were not present in any of the floorplans, all of which depicted as one open space what should have been three separate rooms. Moreover, there were often contradictions among the floorplans, including conflicting dimensions and features placed in different locations. Reconciling these contradictions meant closely collaborating with the client, who provided relatively accurate approximations of actual dimensions, as well as in person visits to confirm the accuracy of the floorplans. Digitizing assistance was provided by Mr. Sanchez, who completed the North buildings and the Grove High School building. 
The reserve study, which was the basis for the assets layer, was also delivered in scanned paper form. The data from these pages were manually entered into an Excel spreadsheet. Year-to-year values used a simple function based on average inflation rate and predicted replacement cost of an asset and could therefore be easily calculated in Excel. The values from the spreadsheet were then joined to the attribute table of the FC and exported as a new FC. Some asset items did not include replacement values as they were deemed effectively everlasting or too unpredictable. In such cases, these values were set to null but, at the request of the client, remain as points in the FC

As the outdoor basemap was created using satellite imagery, it was modified to be more accurate. The building footprints were replaced with polygons created from the georeferenced floorplans and the pathways were modified to line up with the newly placed buildings.

\subsection{Summary}

The conceptual data model describes the day-to-day interactions with the material world represented by the system. The logical data model is a representation of the database as it was implemented. The conceptual model associates property items with the school area. Property items are a generalization of asset and inventory items. Asset items are, in turn, a generalization of safety items. Asset items can be composed of other asset items and are replaced by another version of themselves. The distinction between asset items and inventory items is that inventory items are moveable, not permanent, and in some cases consumable. Inventory items can also contain other inventory items. A staff member or other third party can add, remove, and maintain property items.

The logical data model represents indoor features per the ArcGIS Indoors data model, with a structural hierarchy depicting sites, facilities, levels, units, and details. Indoor FM features have attributes following the Indoors model that place them accurately within the building. A significant difference between the conceptual model and the implemented system is that safety features are separated from asset features to account for differences in their visual representation. The outdoors basemap is entirely separate and stored as a vector tile layer.

Data were largely sourced from the client and were almost exclusively delivered in paper form. The floorplans were manually scanned and digitized with the help of Phil Sanchez, an Esri employee who is an MIR parent. The asset item attributes were also manually entered from scanned documents. The outdoor basemap was provided by $\mathrm{Mr}$. Sanchez and modified to make it more accurate and to fit with the indoor features. 


\section{Chapter 5 - Operations in Desktop Software}

This chapter can be considered the first of two implementation chapters, focusing on map creation on the desktop software side using ArcGIS Pro and the scanning software. Chapter 6 will explore the online component of the GIS. Creation of the map began with scanning and georeferencing the paper floorplans, followed by designing the geodatabase. The geodatabase was designed based on the ArcGIS Indoors data model. The digitizing process took advantage of the digitizing tools and quality control checks in ArcGIS Pro.

\subsection{Preparing and Processing Paper Floorplans}

The data for the campus map were delivered as paper floorplans to be scanned, georeferenced, and digitized as line features into a feature class. A large-format scanner was used to accommodate the oversized floorplan pages. Georeferencing employed a combination of LiDAR and satellite imagery to place two ground control points (GCPs) for each raster image.

\subsubsection{Scanning}

As described in section 4.5, the floorplan data were delivered in paper form as 159 separate documents. They were largely copies of the original documents used in the planning, approval, and construction phases of the buildings. No "as-built" plans were available, meaning that information about changes made in the construction process were not made available to the project. Any changes made in construction not reflected by the floorplans had to be reconciled through physical observation and measurements. For the older buildings, especially the main building, many of the pages had deteriorated so much that they may not survive being handled more in the future. Therefore, it was determined that, for preservation purposes, all pages would be scanned regardless of their utility to the project and included as one of the deliverables.

Scanning took place on a large-format scanner provided by the University of Redlands. In contrast to a flatbed scanner found in most consumer applications, where the sensor moves across a document pressed onto a sheet of glass, large-format scanners use rollers to continuously pull the page across the sensor. This method means that there is virtually no limit to the length of the document being scanned and the amount of time taken to load each document into the scanner is dramatically reduced. The only physical limitation imposed by the scanning hardware is the width of the sensor.

The images were scanned at 500 dots-per-inch (dpi) .tif files to provide adequate quality while keeping file sizes in check. Also to reduce file sizes, the images were scanned with 1-bit black or white color-depth. This also creates perfect contrast between the pixels, easing viewability in the manual digitizing process and optimizing the documents for optical character recognition (OCR). The scanning software automatically assigned the pixel values based on a pre-defined threshold. This threshold can be adjusted by the user, but the default values were determined to be adequate. 


\subsubsection{Georeferencing}

Georeferencing is the process by which spatial references are assigned to the image files to accurately place them on the earth's surface. The process uses GCPs to assign a point from the image to a known specific location on earth. ArcGIS Pro includes tools for georeferencing and allows the user to check for accuracy against multiple data sources including aerial imagery and LiDAR data. Depending on file type, the spatial data can be stored as part of the image, or as a separate .xml file that ArcGIS Pro is capable of recognizing.

The tif files can be added as-is to the project and georeferenced with spatial data stored in separate files. If this method is chosen, the user must be sure to keep the image and resulting .xml spatial data file in the same directory so ArcGIS Pro can always reference the files to place the image. Therefore, care must be taken when viewing and manipulating the files outside of the ArcGIS environment. An alternative is to export each file as a GeoTIFF, which behaves like a normal .tif file, but embeds the spatial data in the file itself rather than referencing it separately. However, this still means that the raster files have to be maintained separately from the rest of the project data (Esri, n.d.-f). Because of these issues, it was determined that the best solution was to import the tif files directly into the working File Geodatabase. This way, the rasters can be manipulated along with the rest of the dataset, lowering the risk of corruption or dropping the spatial reference. Raster files are imported to geodatabases using the Raster to Geodatabase tool found in ArcGIS Pro.

Now, the rasters are imported into the geodatabase, but still lack a spatial reference or location information. The first step in the georeferencing process is to define the spatial reference and its accompanying projected and geographic coordinate system (figure 5-1). For the data creation phase of this project, the State Plane Coordinate System (SPCS) California Zone 5 was used; SPCS CA Zone 5 is highly accurate within the project area of Redlands, CA. The georeferencing tool will automatically default to the spatial reference of the active map frame. It is important that the rasters to be georeferenced have the same spatial reference as the aerial imagery and other sources they are being georeferenced against.

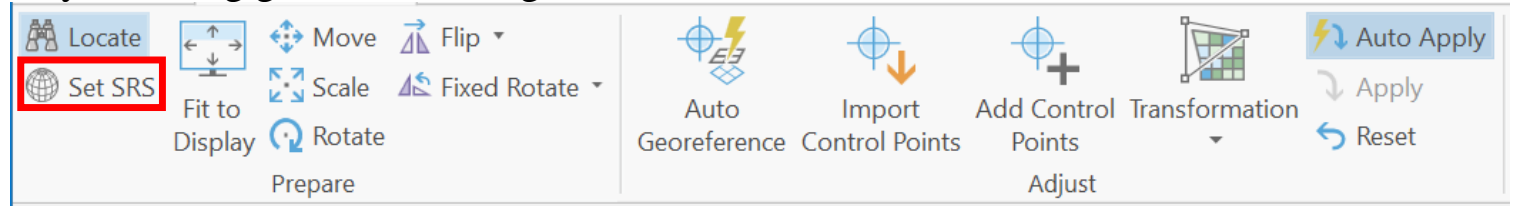

Figure 5-1: Set Spatial Reference in the georeferencing tab

With spatial reference assigned, georeferencing is a simple matter of adding GCPs to locate the image on the earth. When georeferencing, four fundamental transformations are performed: translation, rotation, scale, and skew. As explained in section 2.2.2, it is important to avoid skew, since this will result in distortion in the drawings, meaning that the floorplan is no longer scaled in accordance with the dimensions written on the images themselves. To avoid skew, a maximum of two GCPs are used for any single image (figure 5-2). If less than three GCPs are used, the software will perform the georeference and save it, but it will not store the GCPs to be referenced later. This method is appropriate when working with very large-scale GIS environments where geometric 
distortion is of little concern, but very accurate measurements are of high concern. In other words, the features in the floorplans were already appropriately placed when they were drawn and reflect the buildings they represent within precise specifications. Altering the image through bending and contouring can result in inaccuracies when digitizing.

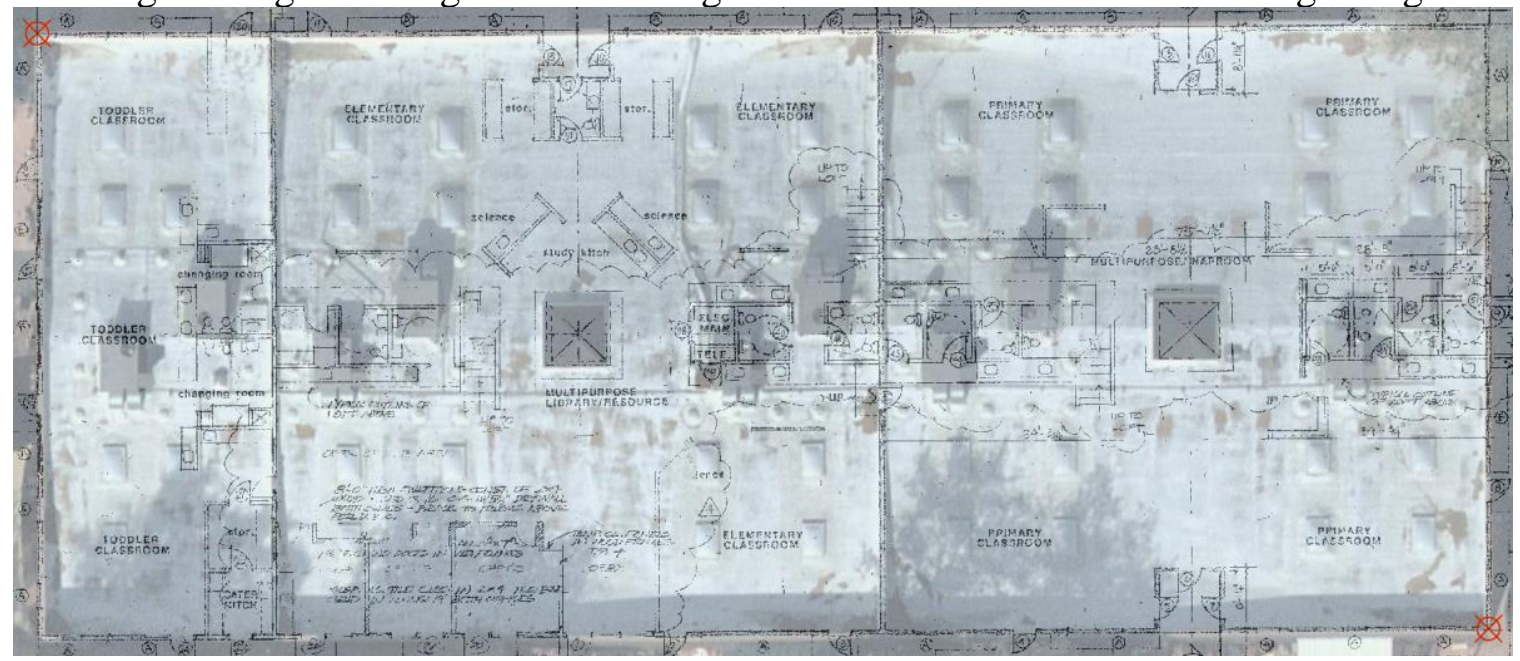

Figure 5-2: Main building floorplan against Esri imagery with GCPs

GCPs were created using the Esri aerial imagery basemap and checked against LiDAR data. The lower-level floorplans were georeferenced first and the upper-level floorplans were georeferenced second based on the lower-level to ensure they lined up well. The GCPs were placed in the extreme opposite corners of the building footprint as indicated by the aerial imagery. While the Esri basemap does use a different spatial reference (Web Mercator), which is projected "on-the-fly" to SPCS, it was deemed appropriate for this project since the data were meant to be used online and were ultimately published in Web Mercator. The GCP locations were confirmed against LiDAR data layers that use the same SPCS coordinate system (figure 5-3).

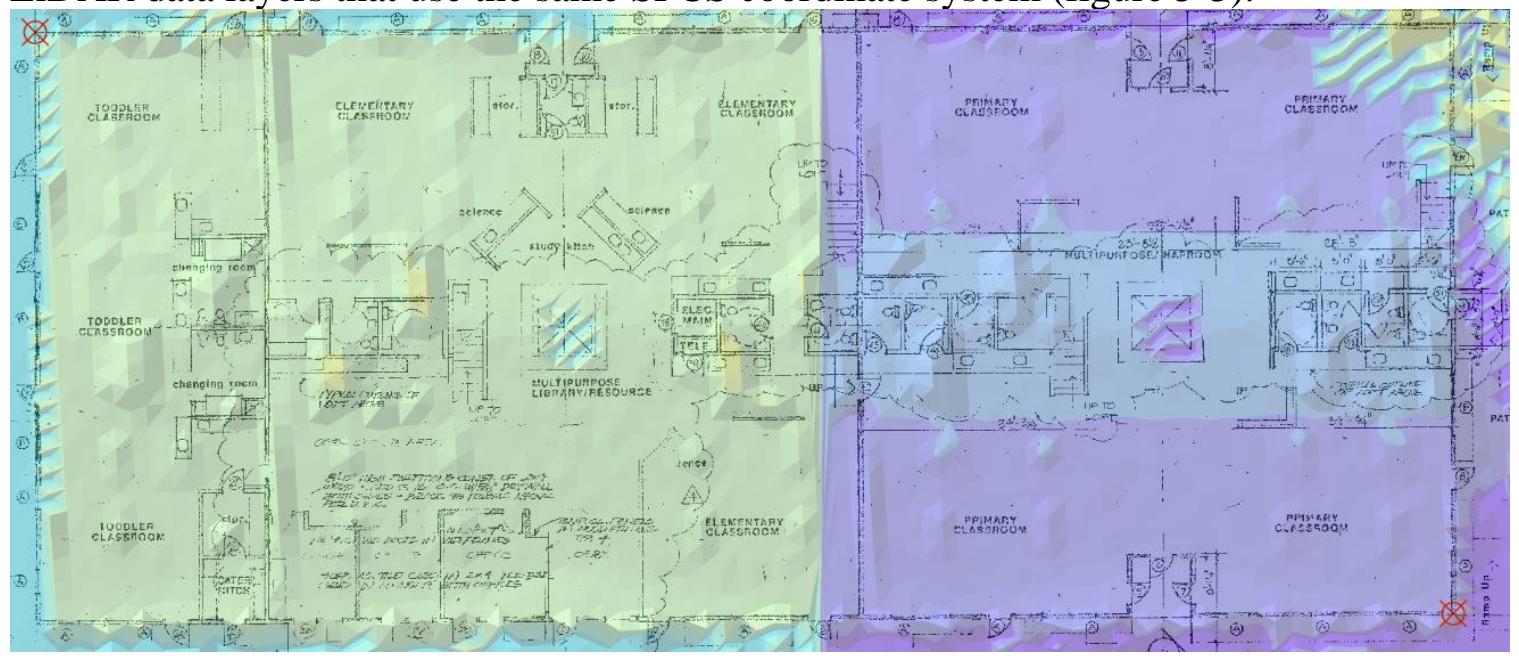

Figure 5-3: Main building floorplan against LiDAR data 


\subsection{Geodatabase Design}

A well-designed geodatabase is a fundamental component of any GIS. The components of this geodatabase are feature classes and their attribute tables, feature datasets, and raster datasets. When creating a new project in ArcGIS Pro, a new geodatabase of the same name is also created. Input data used for this project were first copied to this geodatabase. Indoors feature classes along with their attribute fields were created for the project prior to commencing digitizing. It is important to select the appropriate data type for the field before adding data to it as each field can only have one data type and can't be modified later. Per the ArcGIS Indoors specification, all these fields were of the type Long Integer or Text.

\subsubsection{Fundamental ArcGIS Indoors Feature Classes}

Establishing the ArcGIS Indoors data model is a matter of creating the appropriate feature classes (FCs) and their appropriate attribute table fields. The FCs created for this project were Sites, Facilities, Levels, Units, and Details. Details is the line FC into which the floorplans were originally digitized. The remaining FCs are polygon FCs, with features drawn from the line features previously created in Details. All polygons are bounded by line features except for Sites, which does not represent buildings, but instead represents the entire land area of the campus. These FCs are contained within the Indoors feature dataset and share a common coordinate system. Again, the coordinate system used in the digitizing phase was SPCS California Zone 5, which is converted to Web Mercator upon publishing to ArcGIS Online.

For the floor aware functionality to work in the system deployment, each feature must contain information to locate it in a specific building. Adhering to the Indoors spatial hierarchy, features must be fully contained by other features and reference each other using a unique ID. For example, the Units FC contains fields for UNIT_ID

FACILITY_ID, LEVEL_ID, and SITE_ID, along with the name for each (figure 5-4). Note in the figure that these fields use coded value domains so actual values are not shown (see appendix A for a full list of domain values). The UNIT_ID must be unique within the FC, but the LEVEL_ID and FACILITY_ID are present to reference a specific unit, or room, to the building and level it's located in. Therefore, a unit can only be in one building and on one level. 


\begin{tabular}{|l|l|l|l|}
\hline UNIT_ID & FACILITY_ID & LEVEL_ID & SITE_ID \\
\hline MIR.MAIN.E.L1.Activity_ & Building E & Building E, Level 1 & MIR Main Campus \\
\hline MIR.MAIN.E.L1.OfficeW & Building E & Building E, Level 1 & MIR Main Campus \\
\hline MIR.MAIN.E.L1.Hallway & Building E & Building E, Level 1 & MIR Main Campus \\
\hline MIR.MAIN.E.L1.Lunch_R & Building E & Building E, Level 1 & MIR Main Campus \\
\hline MIR.MAIN.E.L1.OfficeE & Building E & Building E, Level 1 & MIR Main Campus \\
\hline MIR.MAIN.E.L1.Electrics & Building E & Building E, Level 1 & MIR Main Campus \\
\hline MIR.MAIN.E.L1.Storage & Building E & Building E, Level 1 & MIR Main Campus \\
\hline MIR.MAIN.E.L1.RestrooI & Building E & Building E, Level 1 & MIR Main Campus \\
\hline MIR.MAIN.M.L1.Sycamc & Building M & Building M, Level 1 & MIR Main Campus \\
\hline MIR.MAIN.M.L1.Paz & Building M & Building M, Level 1 & MIR Main Campus \\
\hline MIR.MAIN.M.L1.Maple & Building M & Building M, Level 1 & MIR Main Campus \\
\hline MIR.MAIN.M.L1.Wisterii & Building M & Building M, Level 1 & MIR Main Campus \\
\hline
\end{tabular}

Figure 5-4: Section of the Units attribute table showing IDs for Indoors

Details, on the other hand, are usually not located in any one unit, so they only have fields for LEVEL_ID and FACILITY_ID. Also in the Units and Details FCs is a USE_TYPE field. In Units, this records the function of the space such as classroom, restroom, or office. In Details, USE_TYPE notes the use of the specific feature such as interior or exterior wall, window, or plumbing fixture. All FCs also contain a GLOBALID field, which, in contrast to OBJECTID, assigns each feature an ID that is unique between different geodatabases. Global IDs are necessary for data replication and are useful when publishing data to the internet. The ArcGIS Indoors data model is discussed extensively in section 4.2 and section 2.2.3. See appendix A for a technical description of each FC.

\subsubsection{Facilities Management Feature Classes}

The facilities management (FM) FCs created for this project were Doors (contained within the Safety FD), Inventory_Locations (contained within the Inventory FD), and Assets (contained within the Assets FD). All of these FCs are of the type point. Also created was a standalone table called inventory. The Doors FC simply contains a field for door number, which corresponds to physical door numbers posted on the outside and inside of the buildings. The Safety FD that contains Doors is meant in the future to accommodate more safety-related FCs such as fire extinguishers and evacuation zones. Inventory_Locations contains the field LOCATION_ID, which is used as the primary key to the related standalone table Inventory. The related table contains fields for the item's name, ID, type, subtype, quantity, and description. Assets contains fields for an asset's name, ID, quantity, quantity_type, replacement date, remaining life, value, and replacement cost. 
Each of the FM FCs also contain data for the Indoors data model, including the unit ID, level ID, and facility ID and a name for each. Doors only contains level and facility IDs as a door is not necessarily associated with any one unit. These FCs, however, contain a further attribute for location type, which specifies whether the feature is located indoors or outdoors as these features can be in either. In this project, the outdoors are represented by a set of features created in 2017 by Phil Sanchez, an Esri employee and parent of an MIR student.

\subsubsection{Domains and Attribute Rules}

Prior to digitizing, a series of attribute domains and attribute rules were created to ease the digitizing process and ensure accuracy. The domains were used to populate the Indoors attributes as well as the type attributes. It is recommended to use domains for high-volume digitizing, as it ensures typos and other data entry errors will not be allowed to persist through the project. Instead of an empty text box in the attribute table, the user is presented with a dropdown menu and is only allowed to choose a value from that menu. For example, the level ID is unique to each level in each building. Because the digitizing process was mainly conducted one level at a time, the level ID and corresponding names could be pre-selected such that each feature would be automatically populated with the appropriate level ID.

Further, since the level ID is unique unto itself, it could be used to derive the values for other attributes down the Indoors spatial hierarchy. This was accomplished using attribute rules created from Arcade expressions, which is Esri's proprietary expression language. A separate attribute rule must be defined for every field the user would like to auto-populate. Attribute rules can populate fields with standalone values or reference its domain. If the digitizing is happening on the second floor of building A, for instance, that level ID would be selected and could therefore be used to populate the facility and site IDs as well as their names.

\subsection{Digitizing}

Line features were created using the editing tools built into ArcGIS Pro. Where possible, lines were drawn to the exact measurements denoted on the scanned floorplans. When this was not possible, lines were drawn to match the lines on the floorplans. In some cases, mostly in the main building, existing building features were not shown on the floorplans. In other cases, features that were shown on the floorplans were not present in the buildings. This is the result of either minor renovation projects that did not warrant creating floorplans or changes that were made during construction that were not reflected in the floorplans. An example is found in the toddler section of the main building as described in section 5.1.1 in which the floorplans show as one open space what should be three separate rooms. Another example is a bathroom on the north side of the main building that was shown on two separate floorplans, but in reality was removed during a minor renovation project in the early 2000s (figure 5-5). The outside alcove area was replaced with a sliding glass door. 


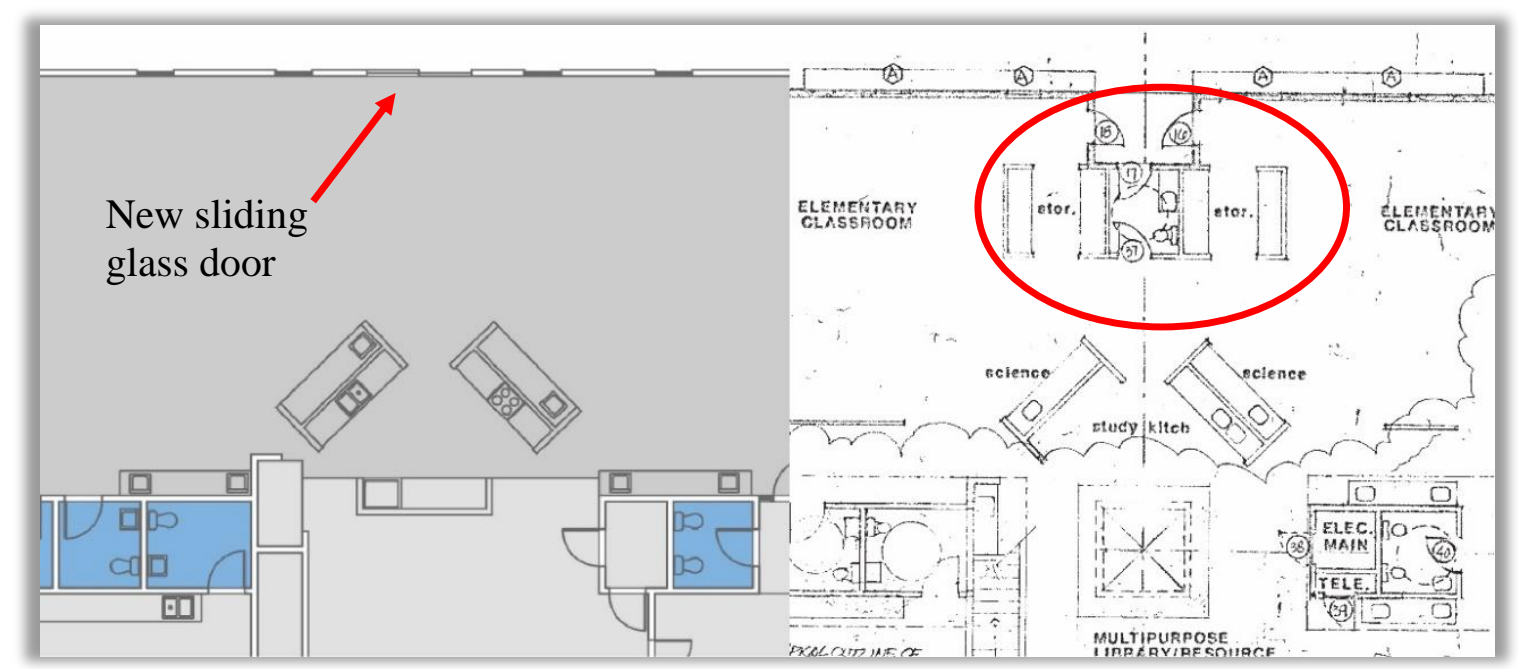

Figure 5-5: Reality versus floorplan depiction indicating removed fixtures

Digitizing assistance was provided by Phil Sanchez. Mr. Sanchez digitized the north buildings (letters A-C) and the Grove High School building (letter D). The north buildings are all identical, save for an office on the East side of building $\mathrm{C}$. Therefore, only one building had to be digitized and its features were copied and placed in the location of the two other buildings. Attribute values and features were then modified appropriately.

\subsubsection{The Reference Grid}

The reference grid was used throughout the digitizing process. In digitizing line features, it was important to ensure that the features, where appropriate, are kept perfectly perpendicular or parallel to each other. ArcGIS Pro includes the ability to "snap" features to other features within a pre-defined tolerance. There is also the ability to specify that lines should be drawn as parallel or perpendicular to another. The reference grid is a uniform grid of equal-area squares, providing a plane on which to create and edit features. The origin, rotation, and grid spacing are customizable and operate independently of the spatial reference. Instead of snapping the pointer to other features, the pointer can snap to the digitizing grid. In addition, new features can be created as perfectly perpendicular or parallel without having to originate from other features.

The reference grid can also assist in quickly approximating measurements. For example, wall thickness across most of the buildings was 6 inches. Setting the spacing on the reference grid to .5 feet allowed for immediate verification of wall thicknesses. Further, distances whose length are to a foot or half foot can be easily drawn by counting cells on the grid. For features that are not oriented cardinally, the grid could be rotated to create features at any angle. The reference grid is shown in figure 5-6 with a red point indicating the origin of the digitizing grid. 


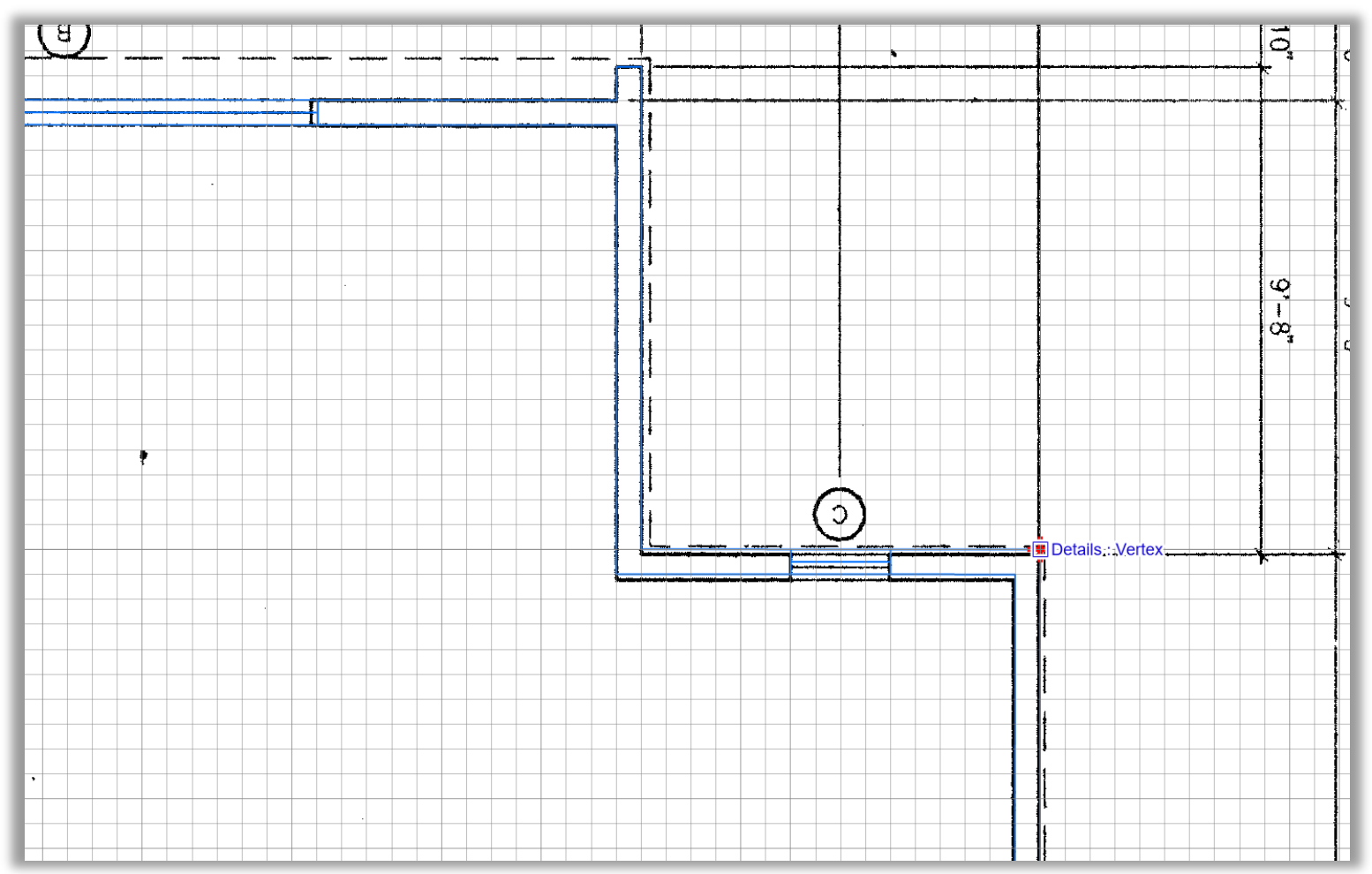

Figure 5-6: The reference grid in the Northeast corner of Building A

\subsubsection{Templates}

Three types of editing templates can be created in ArcGIS Pro: group, feature, and preset, all of which were utilized for line digitizing. Group templates allow the user to create many features from one editing action. For this project, group templates were primarily used in digitizing walls and windows. Because most walls were 6 inches thick, the group template would automatically create two lines with 6 inches of spacing. The user can specify the side on which of the primary line the second line will be drawn. This feature avoided the need to constantly measure the wall thickness or to reposition the reference grid. Windows, in this case, were represented as three lines spaced two inches apart from each other (figure 5-7). The group template could then draw three lines for a total thickness of 4 inches in the window areas.

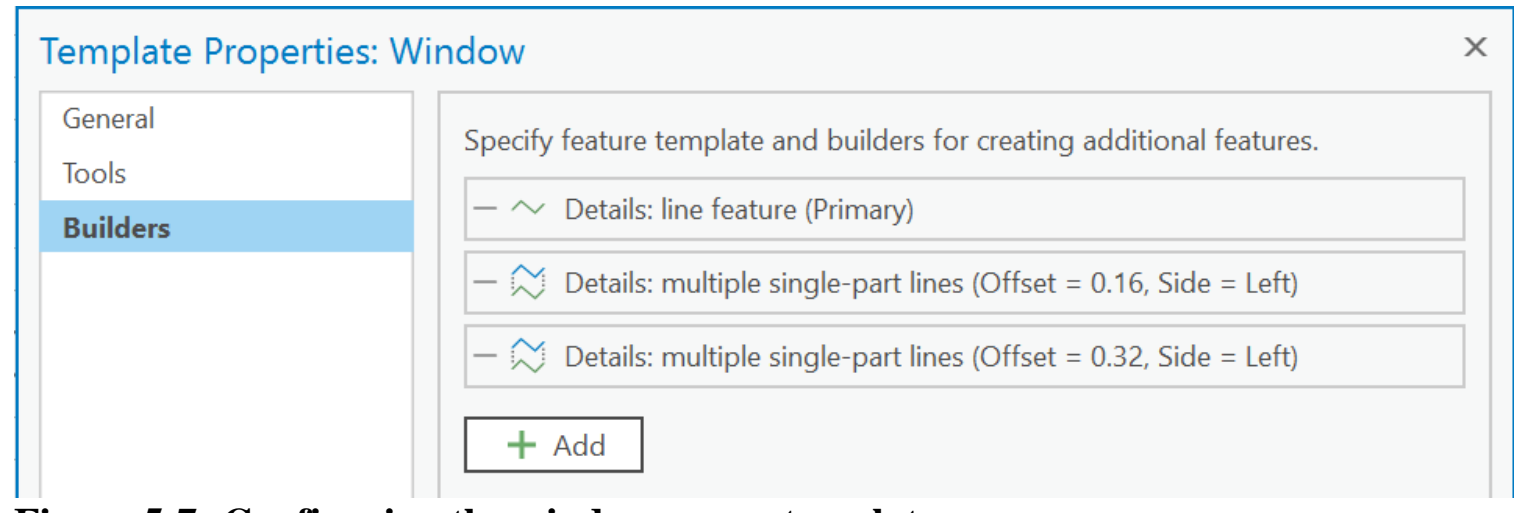

Figure 5-7: Configuring the window group template 
Feature templates are used to automatically populate attribute values during digitizing. In digitizing, the type of line feature was recorded along with the ArcGIS Indoors attributes. The line type can include interior or exterior walls, stairways, windows, doors, etc. Preset templates were arguably most useful for the project, easily recreating complex features with a single click. Preset templates can be added from previously created features. They were primarily used in this project to create doors, sinks, toilets, and kitchen appliances. The example in figure 5-8 shows creating a door using a preset template.

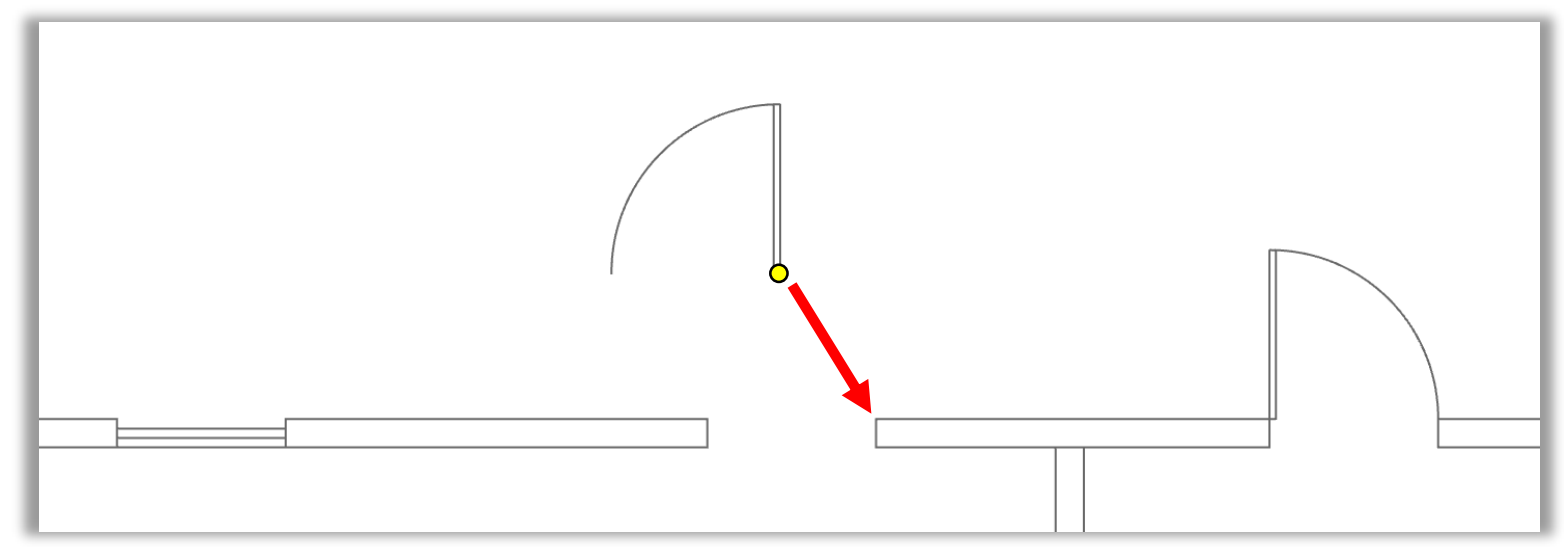

Figure 5-8: Creating door features using a preset template

\subsubsection{Creating Polygons}

The Indoors data model requires polygons in addition to the line features to represent indoor spaces. Polygons were drawn from the bounding lines created by the digitized line features. While tools exist to automatically draw these polygons, such as Feature to Polygon, the lines were ultimately deemed too complex to use these tools. Therefore, the polygons were drawn manually. The site polygon was created from the parcel land cover provided by the city of Redlands. Facilities polygons were drawn from the maximum outer lines of each building in Details to represent the footprint of the building. Level polygons are drawn to represent the footprint of levels with each level on top of the other. Facilities are distinct from levels in that they show the entire area that building occupies whereas levels show only the area that one level occupies. For example, the footprint of a building (the facilities polygon) may include exterior stairways and roof overhangs. The level will only include the wall boundaries of that floor.

For drawing unit polygons, two options exist. The first is to draw polygons so their edge coincides with the edge of the interior wall, thereby excluding the thickness of the wall from the area of the polygon. The second is to draw them so their edges meet in the wall center, which would mean that there is a Unit polygon covering the entire area of any given Facilities or Levels polygon. For this project, the former was chosen to make the polygons' areas as close to the actual useable area of a classroom as possible (figure 5-9). Further, this method made drawing the polygons easier as the center line for each wall would not have to be calculated. Some Indoors floorplans choose to represent walls with zero thickness, which may simplify the digitizing process, but would create a less accurate depiction of the physical spaces (Tekavec \& Lisec, 2020). 


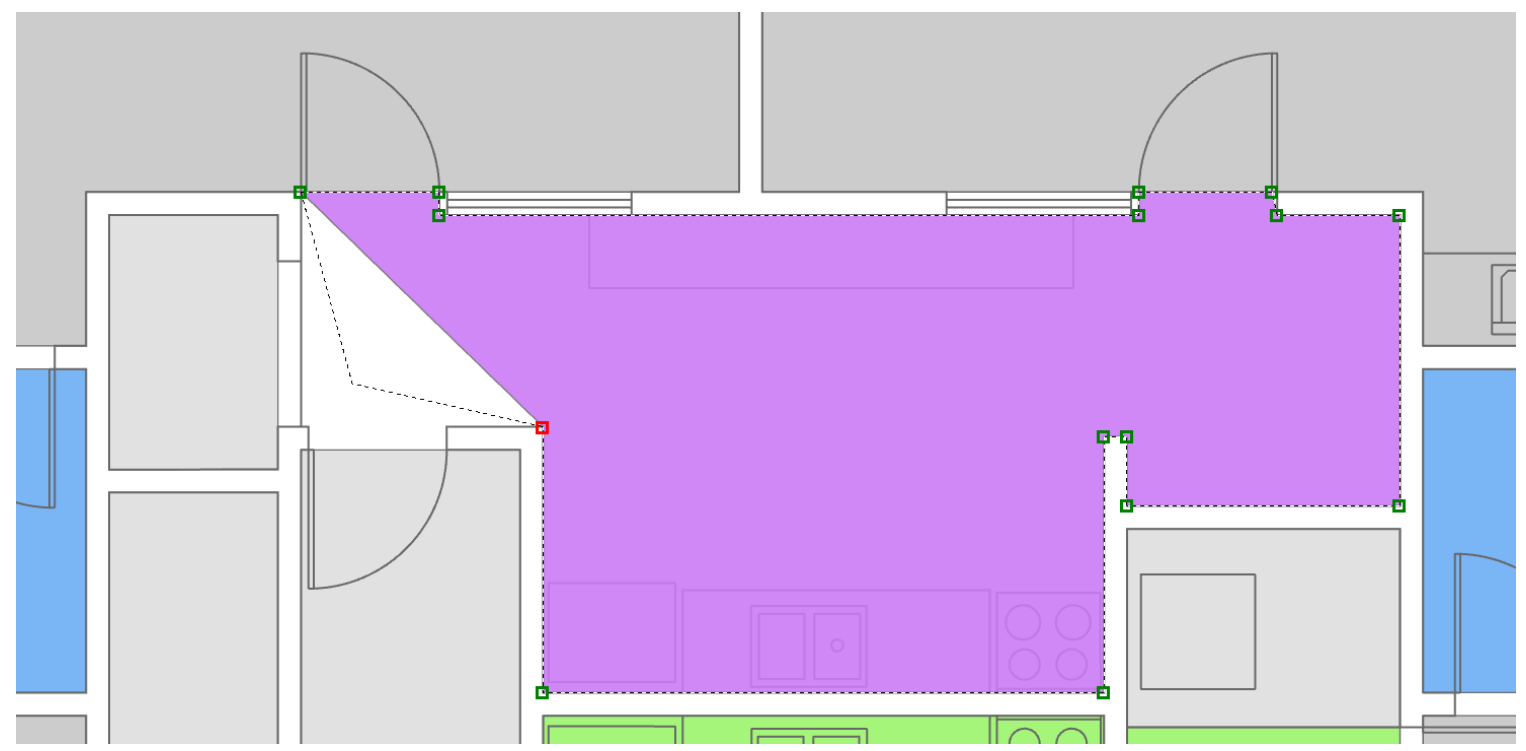

Figure 5-9: Drawing a Unit polygon in the main building

\subsubsection{Attributing}

Where possible, attribute values were populated using domain and attribute rules as described in section 5.2.3 during digitizing and verified later. The IDs for Indoors follow a pre-defined convention of "[organization].[site].[facility].[level].[unit]". For example, a Unit polygon from the Sycamore classroom on level 1 of the main building would have an ID of "MIR.MAIN.M.L1.Sycamore." Therefore, the associated level polygon would have the ID "MIR.MAIN.M.L1" and the facilities polygon would have the ID "MIR.MAIN.M" (Appendix A). In this case, Montessori in Redlands, or MIR, is the organization, and there is only one site, the main campus. The main building is given the building letter $\mathrm{M}$, whereas the other five buildings on the North side of campus are given the letters A-E, beginning with the Southwest building as building A (See Appendix B for a full campus map).

Because all the relevant information for the other attributes is in the Level ID or Unit ID, these were used as the basis for building attribute rules to populate the remaining fields. In the Levels FC, for example, there are attributes for a short level name and a long level name. If, then, the level ID was "MIR.MAIN.A.L1" the name fields would be respectively populated with L1 and Level 1 . Subsequently, the facility ID would be populated with "MIR.MAIN.A", which would trigger the facility name field to have the value "Building A". All these fields also had coded value domains applied to them to provide another level of data integrity.

The other attributes that needed to be populated is the use type of both Unit features and Details features. These also use coded value domains but can't be populated with attribute rules. The Units use type records the normal day-to-day use of the room or space represented by a polygon. A closet, for example, would have the use type "Storage," whereas a classroom would have the use type "Classroom." This is an important field for symbology purposes as the classrooms are color-coded based on their use. The details use type records the use of a specific line feature in the physical structure of the building (figure 5-10). These attributes follow standards for CAD drawings such that they would 
be recognizable to CAD software such as AutoCAD. Most of the features are of the type architectural, as they represent the overall form of the building and its contents, with the only exception being plumbing fixtures, which are of the type plumbing. Architectural features are denoted as "A-Xxxx" and plumbing features are denoted as "P-xxxx".

\begin{tabular}{|l|l|l|}
\hline \multicolumn{1}{|l}{ Code } & Description \\
\hline A-WALL-EXTR & Exterior wall, interior line \\
\hline A-WALL-INFR & Exterior wall, exterior line \\
\hline A-WALL & Interior wall \\
\hline A-WALL-PRHT & Interior Wall, partial height \\
\hline A-AREA-BDRY & Area Boundary \\
\hline A-COLS & Comumn \\
\hline A-AREA-LINE & Area Line \\
\hline A-DOOR & Door \\
\hline A-WALL-GLAS & Glass \\
\hline A-FLOR-STRS & Stairs \\
\hline A-ANNO-TEXT & Annotation \\
\hline NO_VALUE & No Value \\
\hline P-FIXT & Plumbing Fixture \\
\hline A-FLOR-APPL & Appliance \\
\hline A-FLOR-TPTN & Toilet Partition \\
\hline A-FLOR-WDWK Cabinet or Counter \\
\hline A-FURN-PNLS & Cubicle Partition \\
\hline A-FURN-WKSF & Cubicle Work Surface \\
\hline & \\
\hline
\end{tabular}

Figure 5-10: "Details Use" coded value domain

\subsubsection{Topology Checks}

The final phase of the digitizing process is to run topology checks to ensure all features are correct. There are about 3500 single and multipart features in the Details FC. While duplication and automated processes could be employed in some places, most of these features were composed by hand. It is natural for human error to occur when digitizing such a large volume of features, so the intention of the topology checks is to find errors in digitizing. Topology checks are an automated process performed on the geometry of the features to verify they adhere to certain rules. Four topology rules were applied to Details:

- Must not have dangles - The end of a line must touch a part of one other line

- Must be inside Facilities - All Details lines must be inside the Facilities FC

- Must not self-overlap - Lines must not overlap with other lines

- Must be single part - Lines in a single feature may only have one part

The topology is displayed as a separate layer in the contents pane and identifies all the errors as defined by the rules. When an error is identified, it can be manually corrected by the user or the software can attempt an automatic correction. In fixing dangles, for example, the line can be extended, snapped, or trimmed to the nearest line. There are also sometimes exceptions to the rules. Some lines will self-overlap where the walls of two levels are stacked on top of each other. Other features, such as the sinks, will be multipart features. The intention of the topology checks is to find errors in digitizing. The topology can mark these exceptions as such. 
Three topology rules were applied to the polygon feature classes to ensure compliance with the Indoors data model:

- Levels must be covered by feature class of Facilities

- Units must be covered by feature class of Levels

- Units' boundary must be covered by Details

The third rule checks that the polygons perfectly align with the room boundaries created by Details. Errors found under this rule were mainly the result of slivering caused by snapping the polygons to the wrong line vertices.

It was found on several occasions that the topology checks did not identify errors as expected. The example in figure 5-11 shows three dangles where a window has not been connected to the adjoining wall. Each of these lines are stored as separate features, but only the dangle in the center was identified as shown by the red square. As a result, the two un-identified dangles and others like it had to be fixed manually. The cause of this issue was not identified, but upon its discovery, a closer visual inspection was performed to fix any obvious omissions such as this.

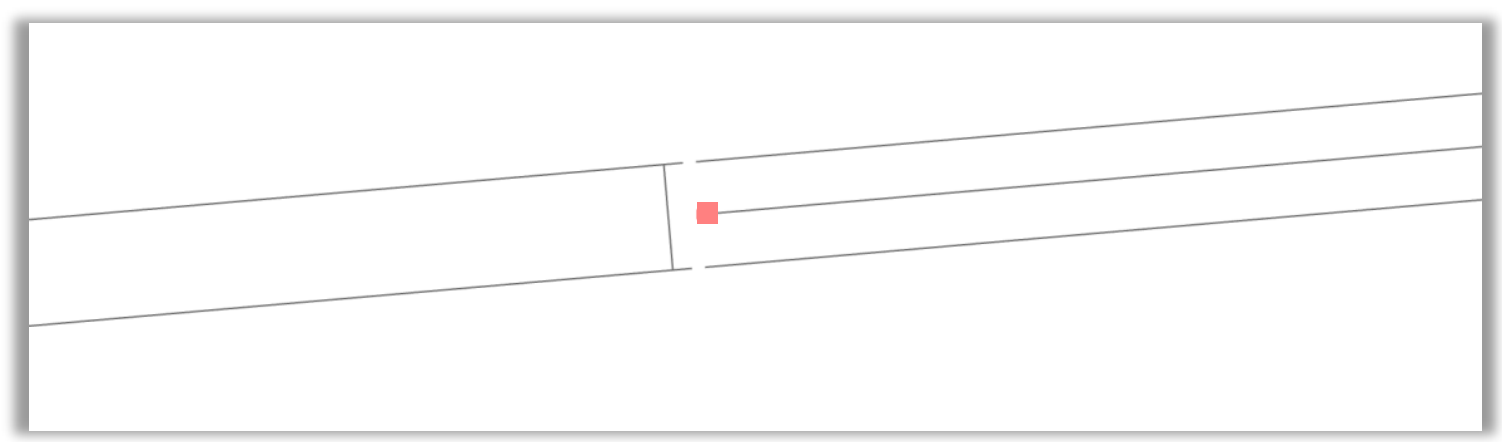

Figure 5-11: Digitizing errors omitted from topology checks

\subsubsection{Creating and Attributing Facilities Management Features}

Facilities management features were created as point features using the digitizing tools in ArcGIS Pro. Attribute values were inputted during the digitizing process. The door numbers were placed at each exterior door and attributed with the appropriate door number. The points were snapped to the boundary of the Facilities polygon for that building.

Inventory points, or catalog points, were added using the Feature to Polygon tool on the Units feature class to create an inventory point in the center of each room. One inventory feature for each room allows for inventory to be tracked at the room level, which was specified by the client. The Inventory_locations FC used the primary key location ID to reference a related table containing the items located in the room. The location ID mainly copies the unit ID except for outdoor locations, where the Unit name is replaced with a different appropriate name. Further points were added for outdoor locations where inventory items such as sheds and playgrounds are kept.

Asset points were digitized at the approximate location of the asset item. Some assets such as skylights have multiple physical items represented by one point and are not necessarily located in a building's interior. It might be more accurate to digitize such features as polygons, but upon consulting with the client, it was determined that these should remain as points for simplicity and ease of use of the system. To show features 
that are not located inside a building, a new Levels polygon known as "roof" was created. Assets such as water heaters are well-suited as point features as they are one object unto themselves and do not take up a significant amount of floor space. Asset points were referred to in the study, from which the data were derived, as components, but it was agreed with the client to refer to them as assets for the project.

The attributes for the assets (outlined in section 4.2 and appendix A) were from a reserve study commissioned by the school in 2008. The values for these assets were delivered as scanned PDFs and inputted by hand into an Excel spreadsheet. At the request of the client, certain changes were made to the values from the PDFs. The study separated the buildings into three "phases," with the main building as phase 1, buildings $A$ and $B$ as phase 2, and buildings $C$ and $D$ as phase 3 . Building $E$ was constructed after this study was completed. As buildings $\mathrm{A}, \mathrm{B}$, and $\mathrm{C}$ are virtually identical, the client requested that building $\mathrm{C}$ be moved into phase 2 , leaving building $\mathrm{D}$ in phase 3 .

The values for a certain asset, then, is the total across all three buildings. The replacement cost of a water heater asset in phase 2 , for instance, would be the total cost of all three water heaters. Since these asset points were to be duplicated across all three buildings, these cost values were divided by three for each of the three buildings and subsequently duplicated. The values were moved from the spreadsheet to the feature class attribute table using the Add Join tool and then exporting as a new FC.

A custom symbology for inventory locations and asset points was created to produce a more user-friendly experience. These symbols consist of easily identifiable icons and are large enough to tap when using the system from a mobile device. They were created in Adobe Illustrator and exported as .svg files for infinite scalability. For example, inventory locations are symbolized as a clipboard (figure 5-12). Asset items are symbolized according to the categories in the reserve study. Electrical items are symbolized as a household electrical plug while plumbing items are a faucet. The full list of symbols is found in appendix $\mathrm{C}$.

Figure 5-12: Inventory location symbol

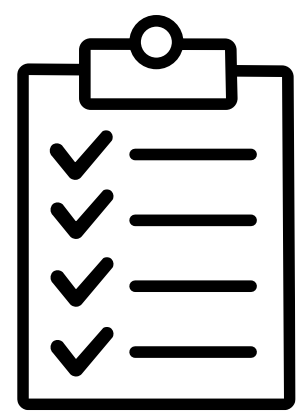

\subsection{Summary}

The implementation of this project begins with scanning the paper floorplans provided by MIR to optimized tif files using a large-format sheet-fed scanner. The relevant files were imported into the project geodatabase and were georeferenced using two GCPs to avoid causing skew in the floorplans. The geodatabase was designed following the ArcGIS Indoors data model with extra point feature classes for the FM features and a separate standalone table to store inventory items. Domains and attribute rules were applied to the attribute tables for quality assurance. Digitizing utilized a series of tools provided by 
ArcGIS Pro for optimal precision. The Indoors features were made up of polygon and polyline feature classes while the FM feature classes were point. After digitizing was complete, topology and manual checks were performed for data accuracy. 


\section{Chapter 6 - Online Deployment}

This is the second implementation chapter, focusing on the creation and operation of the FM system in ArcGIS Online. Feature classes in ArcGIS Pro were uploaded to ArcGIS Online to create a series of Hosted Feature Layers, Web Maps, Web Apps, and Dashboards that comprise the system. The Web Maps were subsequently configured to be used in Field Maps.

\subsection{Maps and Apps in ArcGIS Online}

Map layers in ArcGIS Online are stored as Hosted Feature Layers. These layers can be added to Web Maps, which in turn can be used to create feature-rich tools for users to view and manipulate the data. This project uses Web Apps and Dashboards to provide the client with desired functionality, including viewing and editing inventory items and exploring permanent asset items. Web Maps can also be configured for use in the Field Maps mobile app, which provides much the same functionality. Maps and geodatabases created in ArcGIS Pro can be directly uploaded to ArcGIS Online.

\subsubsection{Uploading to ArcGIS Online}

There are a few ways to get data created in ArcGIS Pro and other desktop applications to ArcGIS Online. One is to upload the geodatabase in a .zip folder, which will then be converted to a Hosted Feature Layer. Another more convenient method is to publish the layers of a map directly from ArcGIS Pro using the Share as Web Map tool. This method was used for the majority of the project except under certain circumstances such as adding data from other sources. ArcGIS Pro allows the user to sign into their ArcGIS Online account and view and manipulate their online content directly in Pro. This content can be accessed from the Portal tab on the catalog pane in ArcGIS Pro. Share as Web Map will create two components in ArcGIS Online: a Hosted Feature Layer and a Web Map with the layers. The alternative tool, Share as Web Layer, will only create a Hosted Feature Layer containing the layers of the active ArcGIS Pro map.

There were a few occasions where sharing failed after repeated retries and restarts. The error code provided was a nondescript error pertaining to a bad URL. The issue was narrowed to a few long integer fields in the Units feature class that triggered a bug related to domain values. The issue was replicable by removing and adding the domains to these fields. Interestingly, the Esri website shows the error as related to ArcGIS Server, not ArcGIS Online (Esri, n.d.-e). The domains were removed from the offending fields and the map was republished. These domains were then re-added to the layers after publishing to ArcGIS Online.

It is important here to also note certain compatibility limitations between ArcGIS Pro and ArcGIS Online. This project utilized attribute rules to automatically populate attribute values based on the values of other attributes. ArcGIS Online does not support attribute rules and any that are included in the ArcGIS Pro project are dropped during the upload process. The functionality of attribute rules can't be directly replicated, but a workaround using a Notebook script to update values can be applied. Additionally, ArcGIS Online uses Web Mercator across its default basemaps. Maps frames must be set 
to Web Mercator before uploading with a default basemap or the process will fail. To use a Web Map in another coordinate system, the analyst can author their own basemap. The layers themselves can still be in their chosen coordinate system regardless of the basemap spatial reference. It is useful to leave the layers in their original coordinate system in cases where editing needs to be performed using accurate measurements, as is the case with this project.

\subsubsection{Web Maps}

Three Web Maps were created in ArcGIS Online after their layers were published in ArcGIS Pro. The first, called Safety, is the Web Map to serve as the basis for emergency first responders and as a general campus reference map. Currently, this map has labels for the classrooms and a separate layer for door locations labeled with the door numbers. The second map is for inventory and contains the indoor and outdoor basemap, the inventory points, and the standalone table for recording inventory items. The third map is for asset management points and includes the indoor basemap, outdoor basemap, and the asset management points.

\subsubsection{Web Apps}

Two Web Apps were created for this project: one for inventory and another for assets. Web Apps are created from existing Web Maps and are customizable with a variety of out-of-box widgets using the Web App Builder in ArcGIS Online. Widgets can include editing tools, summary statistics, querying, and more. The Web App Builder provides a user-friendly interface for creating apps, including offering a variety of themes depending on the use-case of the app. The Launchpad theme, for example, shows widgets as icons in the bottom of the screen, and presents the user-interface (UI) of the widget in a separate popup. The Dashboard theme (distinct from the ArcGIS Online product Dashboards) shows the UI of the widgets as tiles around the map. Both Web Apps in this project used the Tab theme, which displays widgets to the left of the map, allowing the user to click between them.

The Web App Builder is reasonably powerful but is limited to the widgets provided out-of-box by Esri. One widget the Web App Builder lacks is a floor picker to automatically filter floors based on ArcGIS Indoors data, although this feature does exist as a custom widget (Esri, n.d.-c). Custom widgets utilize the ArcGIS API for JavaScript and are inserted by modifying the source code for the App. This widget would have been useful to the project; unfortunately, modifying the source code of a Web App requires the app to be hosted on an ArcGIS Enterprise server. Enterprise is a modified version of ArcGIS Online that runs on servers managed by the organization, rather than on cloud servers managed by Esri. An ArcGIS Enterprise deployment provides valuable customizability but adds cost and complexity, requiring knowledgeable IT staff to manage. One possible reason Esri does not include a floor picker in the Web App Builder is that Web Apps are not strictly intended for displaying indoor features. A separate product called Indoor Viewer, which includes a multitude of indoor tools, is available to organizations that purchase the additional ArcGIS Indoors license.

With the inability to include the floor picker widget, the workaround was to use a Group Filter widget to filter based on the level name (Figure 6-1). Group filter can query 
based on a single value across multiple layers. Since all layers have a value for level name, this was the field that was used. If the user wants to show outdoor locations, they would set the floor filter to no value, since outdoor locations have null values for indoors. This behavior was chosen at the request of the client. If, in the future, that client desires a different behavior, like showing outdoor locations when level 1 is selected, they can simply populate these fields.

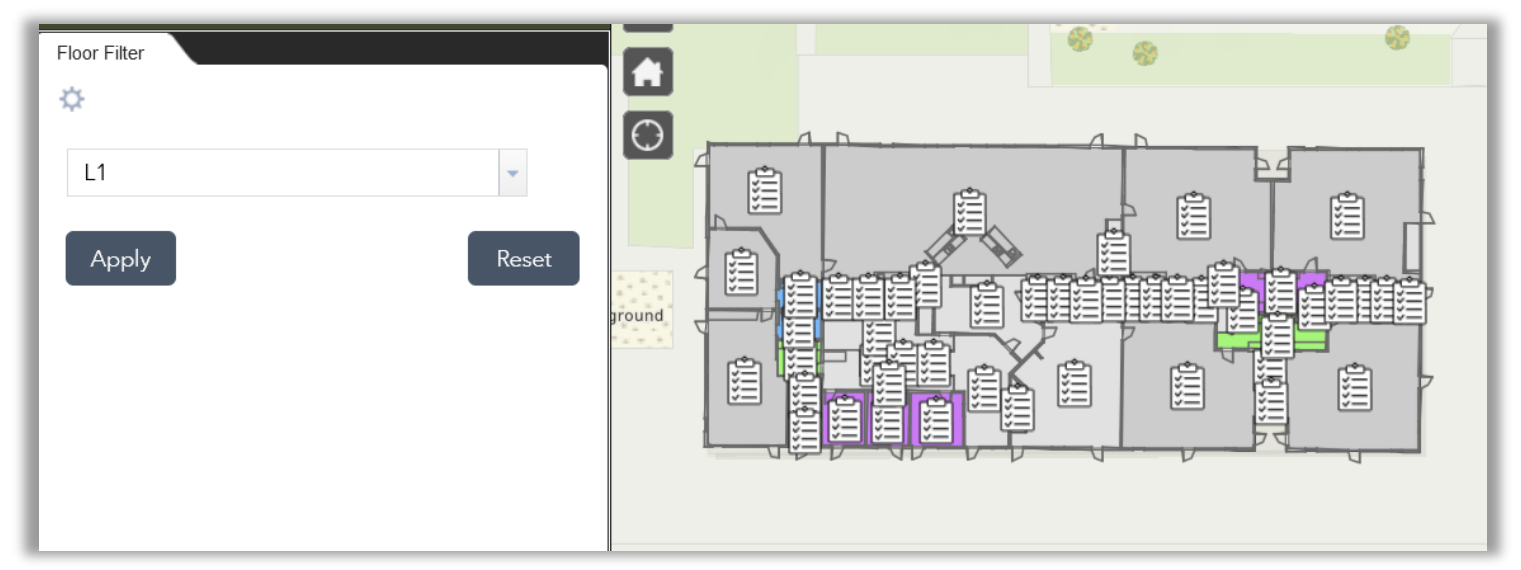

Figure 6-1: Floor filter in the Inventory Web App. Set to level 1

Other widgets in the Web Apps are Legend, Layer List, and Edit. Legend shows each layer and its symbology. Layer List also shows the layers and their symbology, but has the added functionality of toggling on and off the individual layers and viewing the attribute table for each layer. In the Inventory app, the standalone inventory items table is also included. If the user wants to edit the inventory locations, they can use the Edit widget. In the Inventory Web App, the user can add, delete, and move inventory locations. Before adding new inventory locations, the user must first create a new location ID domain value to the inventory locations layer. This is to prevent inventory locations having duplicate location IDs or inventory items having no location ID. Domain values cannot be added or altered from the Web App, they must be added from the settings menu of the layer.

When a user clicks on an inventory location, they are presented with a popup form to change the attribute values of the point. They can also view and edit the inventory items associated with that point (Figure 6-2). The user must populate the inventory item entry with a location ID. To move an item to another location is a simple matter of selecting a new location ID from the dropdown. Edits persist after the user clicks the save button at the bottom of the popup. Editing Asset points in the Assets Web App behaves in much the same way with the omission of a standalone table. Features from the indoor and outdoor basemap layers are not editable in Web Apps and shouldn't be edited in ArcGIS Online at all. 

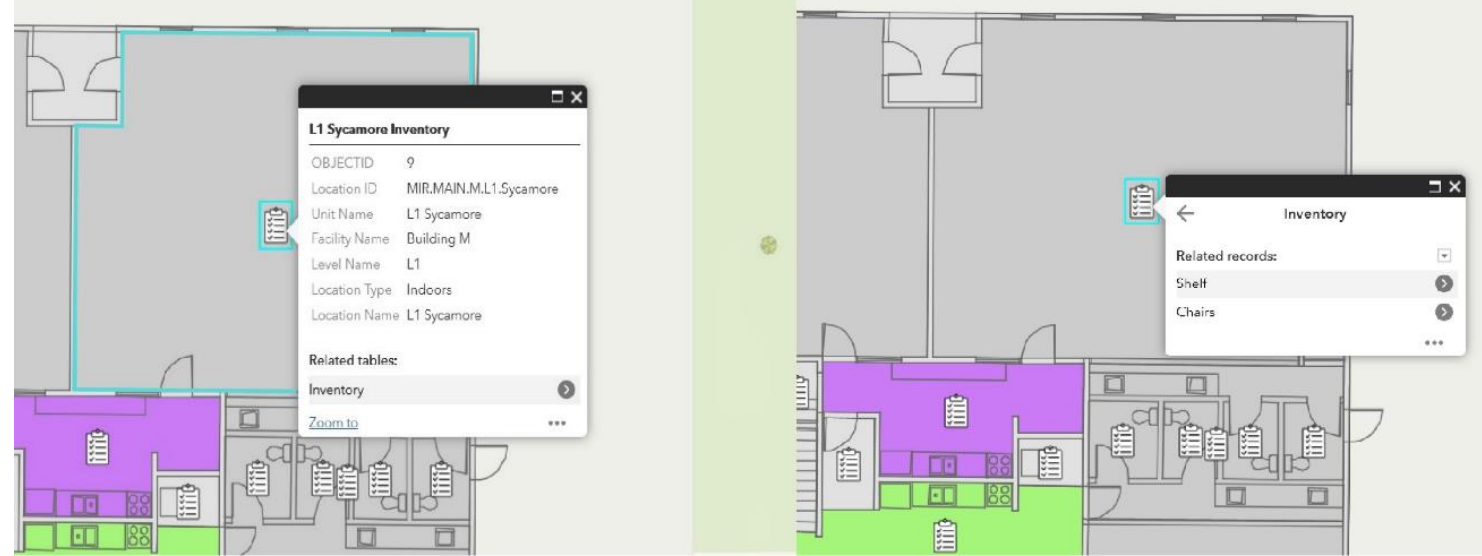

Figure 6-2: Viewing inventory locations and their associated items

\subsubsection{Dashboards}

Dashboards in ArcGIS Online are meant to present relevant information about map data rapidly to the user. In the traditional implementation of a dashboard, the map pane is surrounded by one or more elements and a header above. This project includes two Dashboards: one for permanent assets and another for the emergency first responders. The Assets Dashboard displays a map of all the asset points. In the header, the user can control a series of category selectors to choose between indoor or outdoor features, pick the building floor, and filter assets by their remaining life (figure 6-3). It is necessary to choose between indoor and outdoor features because the floor filter would otherwise filter out indoor features when a floor is selected. When launched, the default view for the Dashboard is to show outdoor items. With indoor items selected, the user should choose the floor to display in the buildings. The category selectors will allow the user to simultaneously select outdoor features and a floor, which would mean there would be no assets to display. This is due to functionality limitations in the category selector. If would be useful to, for example, have the floor picker only be visible after indoor features have been selected.

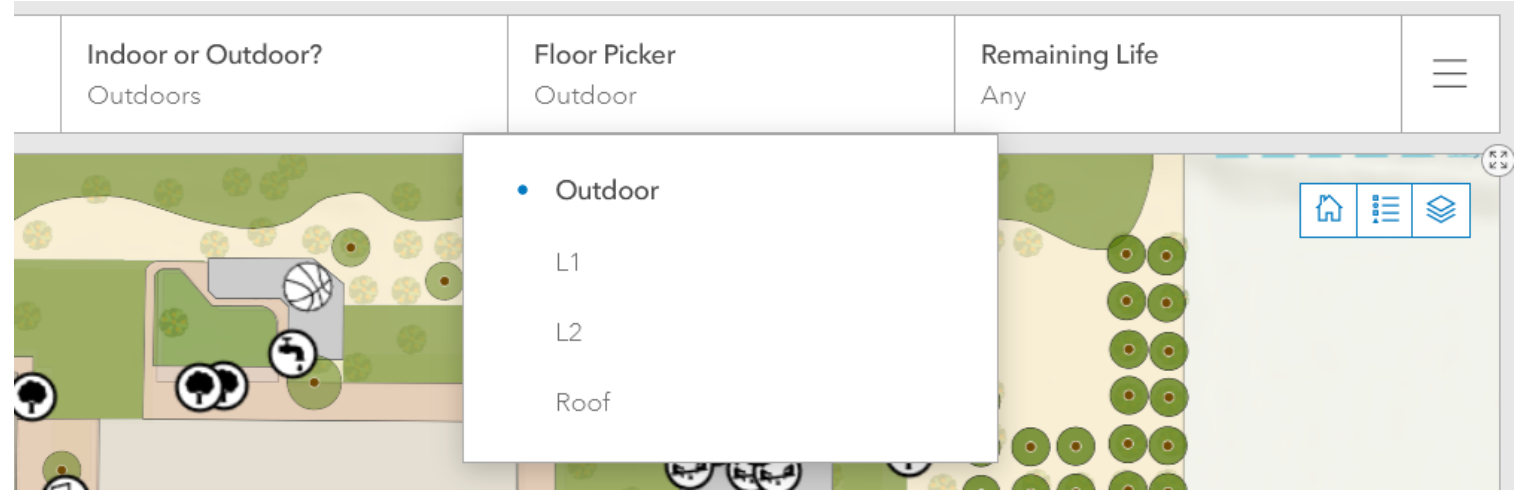

Figure 6-3: Category selectors with floor picker dropdown selected

The remaining elements in the map surround the left and bottom edges of the map pane. The left side includes a legend and a list of asset items. Tabs in the list element allow for toggling between showing only the selected assets or list all the assets. When a 
user clicks on a list item, the map pans to the item, flashes it, and displays a popup with the relevant attribute values. The bottom row displays three elements (figure 6-4). The first is a series of three tabbed lists showing those items that need to be acted upon: this year, are overdue, in one year, or in 2-5 years. The second element shows a count of the items that should be acted upon this year and the third element is a bar graph with the count of items that should be acted upon between 0 and 5 years from the current date.
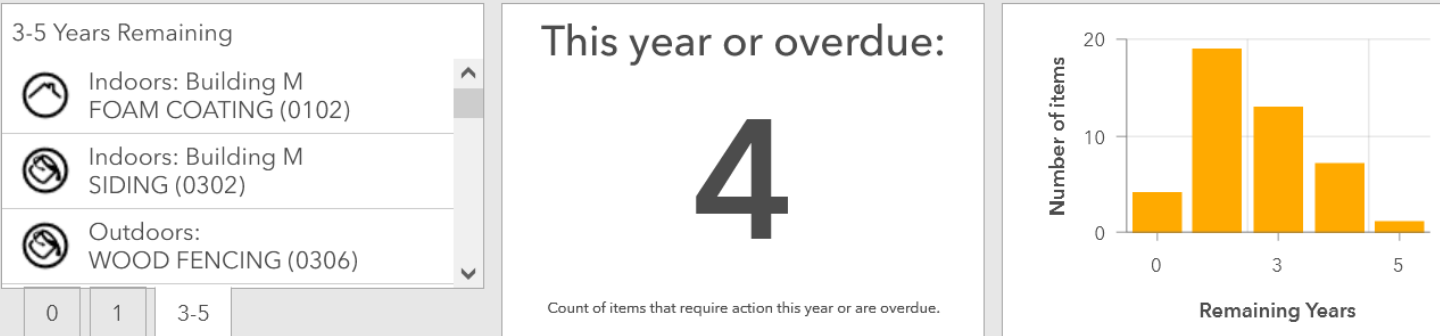

Figure 6-4: Three elements below the map frame in the Assets dashboard

The Safety Dashboard provides a very simple view of campus which shows door numbers and classroom labels on top of the indoor and outdoor features (figure 6-5). The header bar has two category selectors that allow the user to jump to specific buildings and select the level to display. An element to the right provides the user with a list of Units (rooms) in the selected building. The user can tap on Units in the list to flash it. In this case, it was possible for the category selectors to behave as cascading filters where the first selector filters the options for the next. In selecting a building, the list only shows the units in that building. Selecting a level shows only the units on that level. The intent of this dashboard is to provide valuable location information in only a few steps. First, find the building; second, find the room; third, find the door.

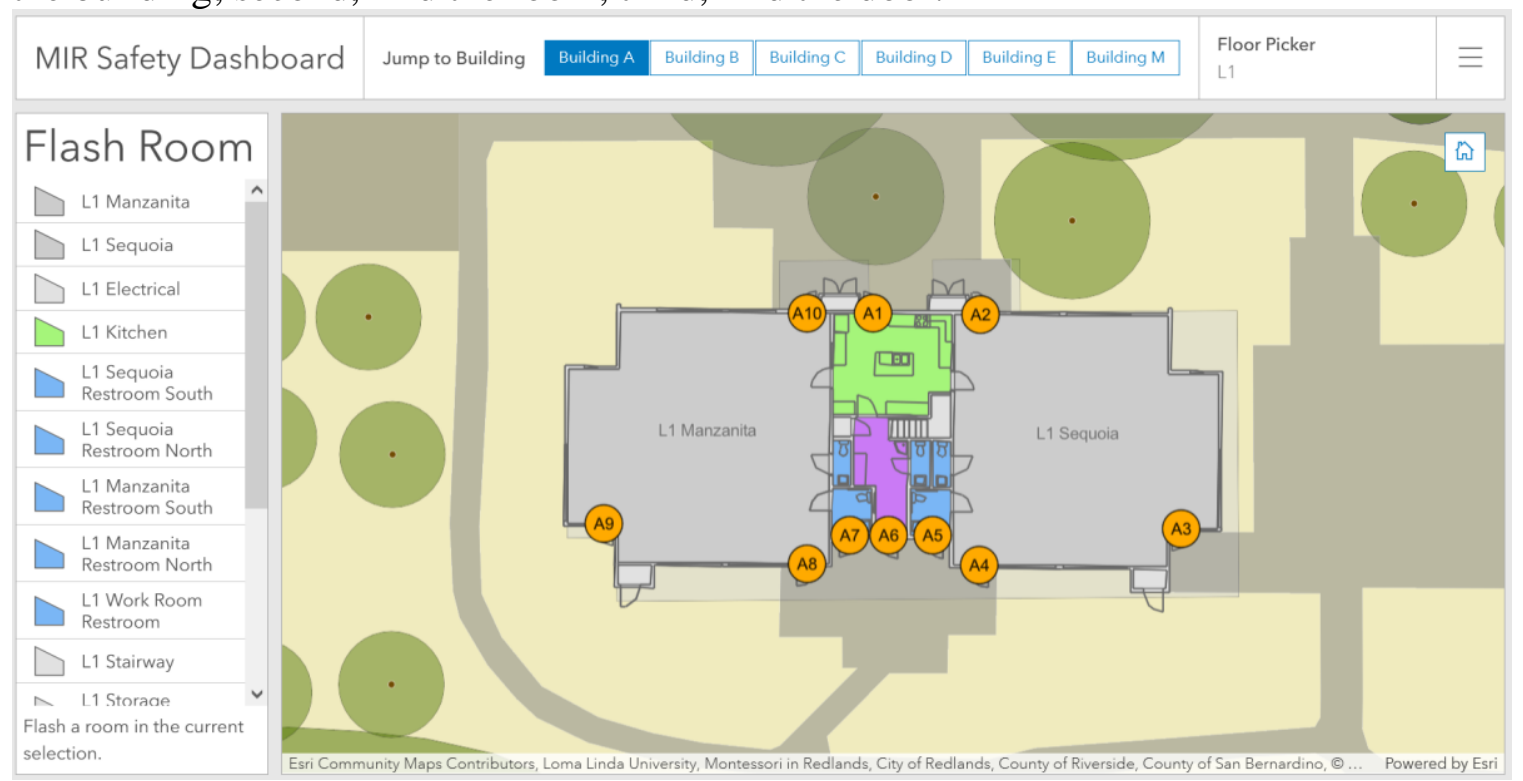

Figure 6-5: Safety Dashboard showing Building A 


\subsection{Field Maps}

Field Maps provides that same general functionality to that of the Web Apps discussed in the previous section. Users can pan around maps, add or update features, view or edit attributes and related tables, and even select between floors. Field Maps is the only component of this system that enables automatic floor filtering (figure 6-6). When a map is added that contains Indoors information, a selector is displayed to change the floor that is being viewed. When using Field Maps with Indoors data, the app will only display the building in the centermost part of the screen regardless of the map extent. If, for example, buildings $\mathrm{A}$ and $\mathrm{C}$ are within the map extent and only building $\mathrm{A}$ is shown, the user must pan to the center of building $C$ to display it (figure 6-6). Similarly for outdoor points, the user must center the points on the screen to display them.

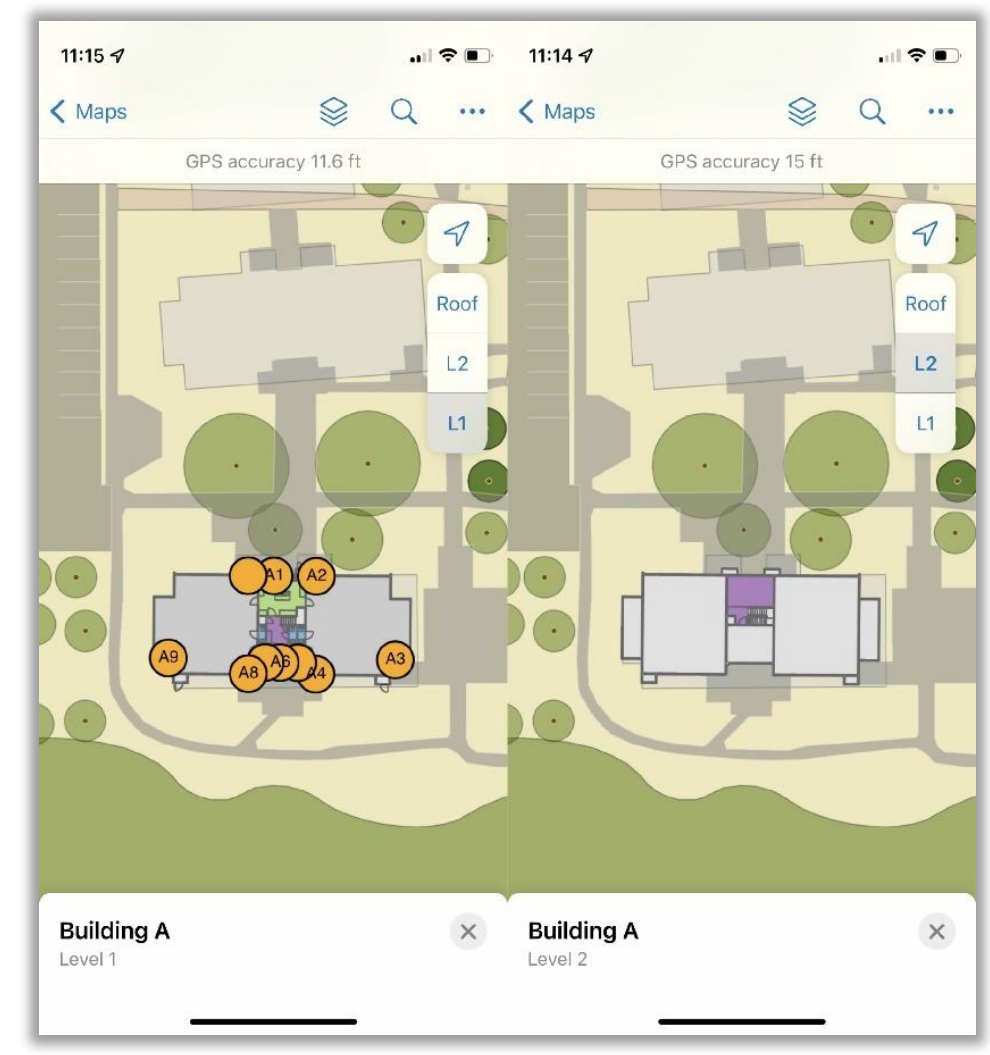

Figure 6-6: Field Maps showing Safety (Building A level 1 and 2)

The setup of Field Maps takes place in a web interface, not on the app itself. The content tab contains the form builder where customized forms were created for the layer being edited (figure 6-7). Fields can be added in whatever order the user wishes and is not dependent on the original configuration of the popup or the attribute table. The user can also choose which fields are to be added to the form or choose to disable editing of a layer altogether. By default, the form follows the layout of the popup configured in the Web Map editor. Fields can also be set up with "conditional visibility," meaning that field will appear only if certain values in another field are selected. When adding or editing inventory items, for instance, the item type (desk, chair, electronics, etc.) appears 
as a list of values from a domain with an option for "other." When "other" is selected, a new field appears prompting the user to manually enter the item's type.

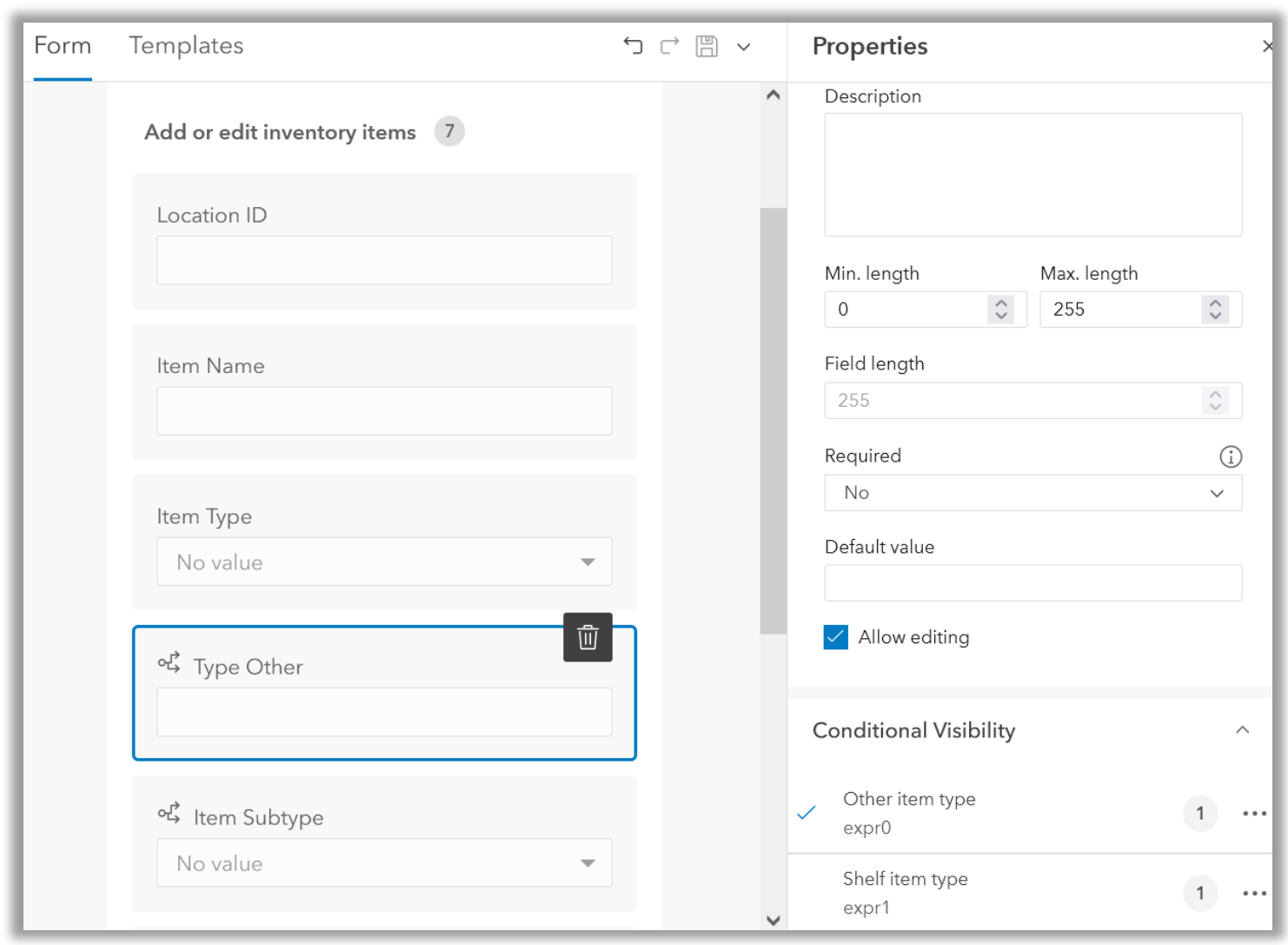

Figure 6-7: Field Maps configurator editing Inventory Items

When an FM point is selected in Field Maps, its popup is displayed as configured in Map Viewer. Popups for the indoor features are disabled in the Web Maps to avoid (a) presenting superfluous information to the user and (b) accidental edits. If the popup for a feature is disabled, it will not be selectable in Field Maps. As in ArcGIS Online Map Viewer, controls in Field Maps are not precise enough to make edits to the indoor features. With the popup displayed, the user can review the attribute information, including any attached images. If the feature has a related table, a button appears to view related content. In this system, inventory locations have an option for related content, which shows an area's associated inventory items (figure 6-8). 


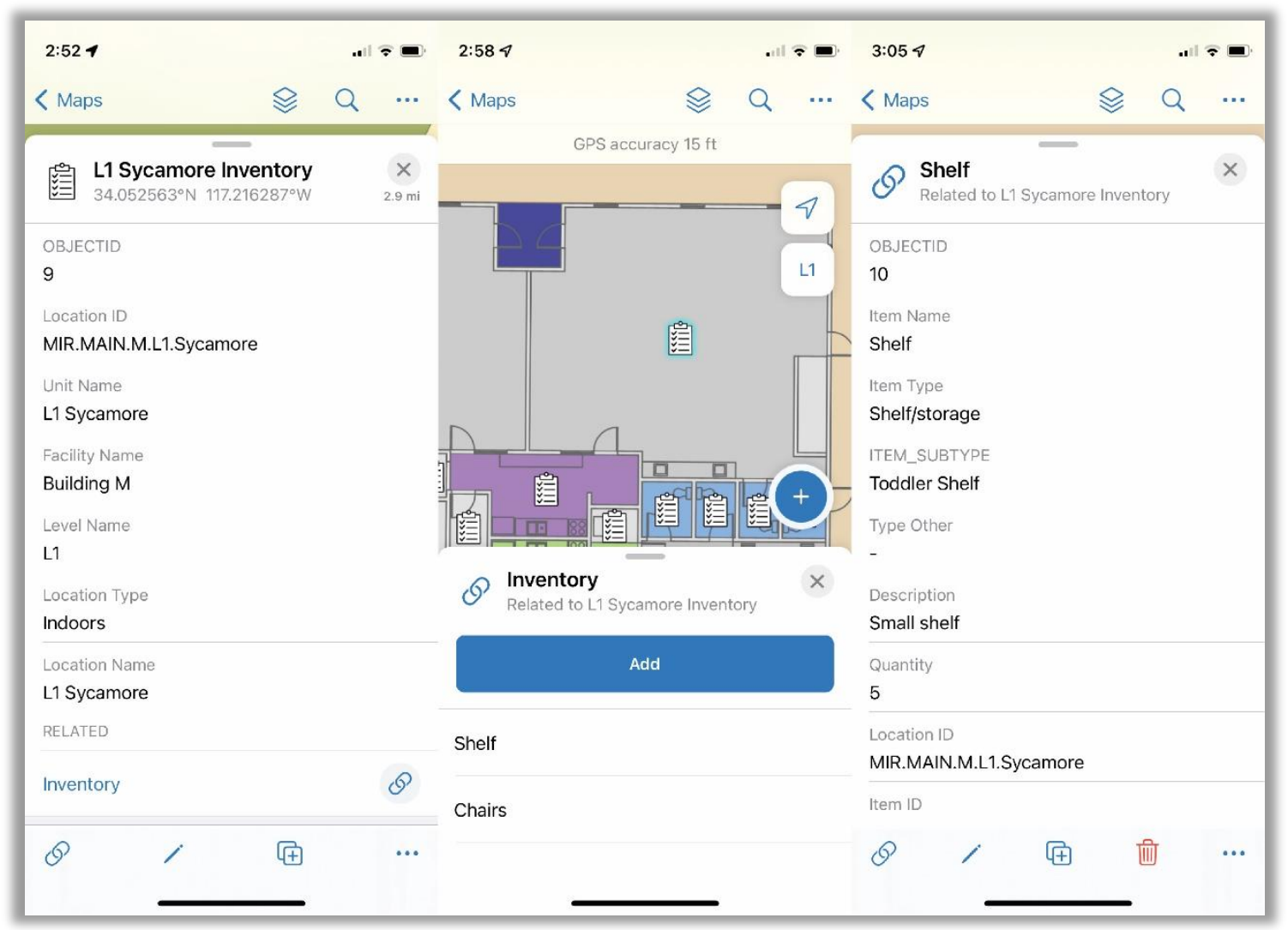

Figure 6-8: Inventory location, related items, and item details

Editing features and their attributes in Field Maps works in much the same way. When a feature is selected, the user can choose to edit, which presents the form configured previously. The user can add, edit, or delete features based on the privileges they have been assigned. Features and related table entries can also be duplicated and placed in another location.

\subsection{Summary}

To create the online system, Hosted Feature Layers were published to ArcGIS Online directly from ArcGIS Pro. This process created four Hosted Feature Layers for Indoors, Safety (for first responders), Inventory, and Assets. The outdoor map was also published as a Vector Tile Layer. The indoor and outdoor layers were added to each of the three Web Maps created, along with the relevant FM layer. These Web Maps were then used to build two Dashboards and two Web Apps. The Dashboards are for Safety and Assets (view only) while the Web Apps are for Inventory and Assets (editing). The Web Maps were then configured for Field Maps to bring the system into a mobile-friendly environment. 


\section{Chapter 7 - Results and Analysis}

This chapter describes the client's needs and the components that fit each. It also discusses that changes that had to be made for the design of the system to function with the chosen platform. Several methods were tried, resulting in some that worked.

\subsection{Results}

This project created an online system to meet needs identified by the client in initial meetings by creating a system to (a) manage inventory, (b) track online assets, and (c) provide high-quality campus maps to first responders. It was vital that every aspect of the system could be used through a web browser or mobile app. In a web-browser, the system is accessible through ArcGIS Online while the mobile interface is provided by Esri's Field Maps. The system is hosted in the cloud on an MIR-owned ArcGIS Organizational License. Getting the input indoor data from paper floorplans into a geodatabase took several iterations to develop an efficient and accurate workflow. Publishing from Feature Classes to ArcGIS Online also presented a series of issues.

\subsection{Analysis of Outcomes}

While the deliverables satisfied the client's needs, issues were encountered along the way. This section discusses such issues and describes the solutions that were found.

\subsubsection{Georeferencing Analysis}

In georeferencing, the floorplan rasters were placed to cover the footprint of the buildings. As this system is not meant to serve any functions requiring highly accurate building placement, the floorplans were placed such that they appeared visually correct. Because it was important to avoid skew in the floorplans (see sections 5.1.2 and 2.2.2), only two ground control points (GCPs) were selected for each floorplan. However, for a first order georeference to be saved by ArcGIS Pro, three GCPs are required. Placing two GCPs meant that the georeference would not be saved by ArcGIS Pro. The location and spatial reference would be saved, but not the GCPs themselves. This resulted in floorplans occasionally losing their location and having to re-georeference. Fortunately, after digitizing the building boundaries, the floorplans could be georeferenced to feature vertices, rather than imagery. Attempting to georeference with three GCPs resulted in obvious misalignment between the features and the rasters, resulting in having to constantly double-check measurements.

\subsubsection{Digitizing Analysis}

Digitizing was a manual process, drawing each line segment individually. The georeferenced floorplans mainly served as guides, with lines drawn based on inputted measurements. The floorplans included most, but not all, of the measurements needed to create accurate lines. Floorplans were mainly lacking in interior wall measurements, although most of the exterior measurements were provided. Indeed, Phil Sanchez 
encountered similar issues in his work digitizing buildings A-D. Other measurements, such as the widths of doors and windows, were shown on other sheets in the building plans. Figure 7-1, for example, shows a kitchen and office area in the main building without any measurement provided. Some of these measurements could be inferred. In cases where they could not, physical measurements were taken by Ellen Camarillo, the MIR facilities coordinator, or visually approximated. Similarly, door and window spacing measurements were not noted anywhere in the provided documents and were approximated.

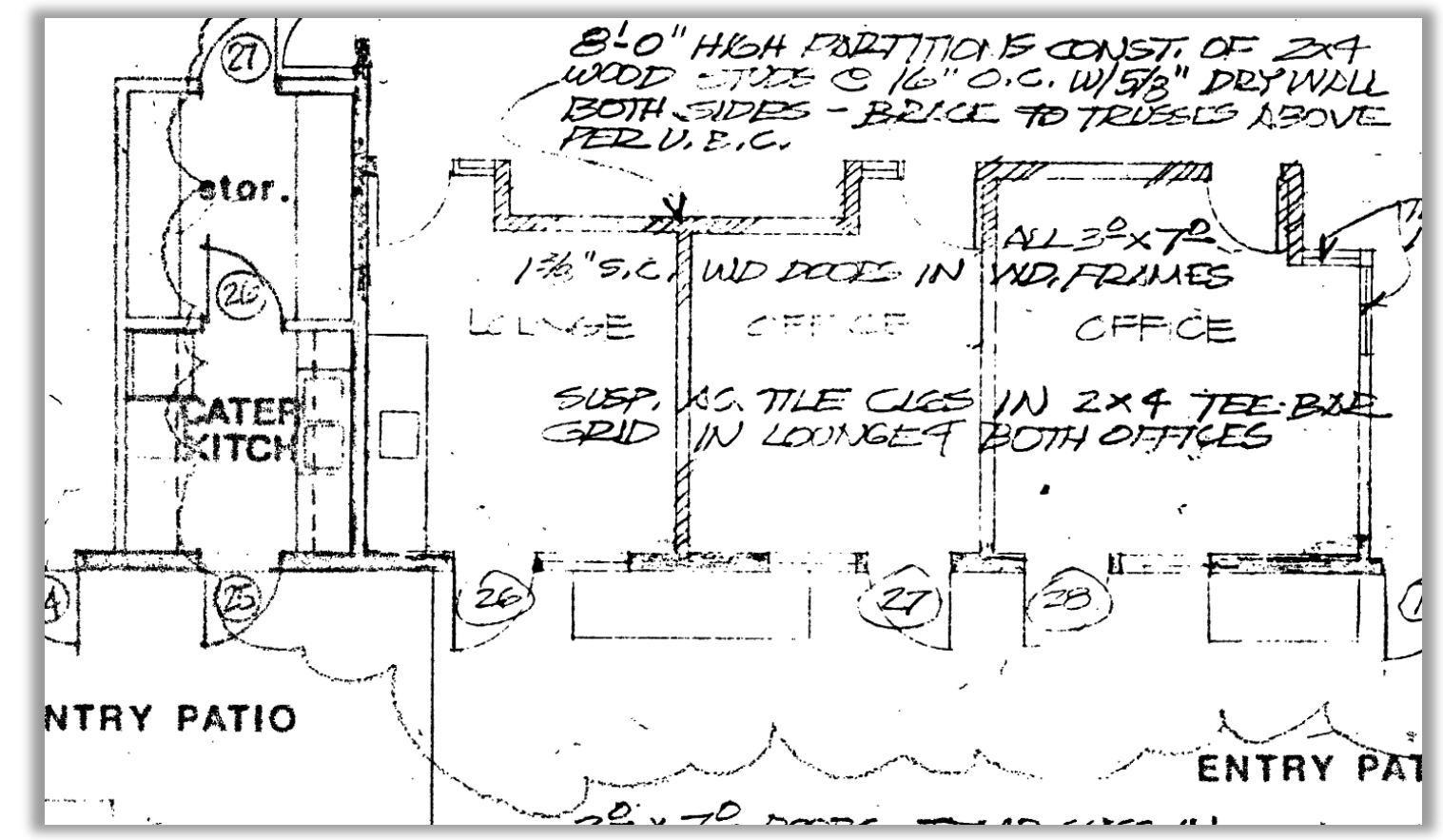

Figure 7-1: Section of Main Building office and kitchen area

In an attempt at efficiency, the first digitizing workflow began with drawing the outline of the building and adding detail by splitting and creating new vertices. Instead, these full-length lines were used as a reference with the actual features, including doors, windows, and their separating walls placed individually. All areas that included measurements were placed before those without, which allowed for a more accurate approximation. It also ensures that the measurements can be verified before adding more features. If a line was digitized inaccurately, fixing that measurement would require shifting all the interconnected features. Correcting these errors used much of the time taken for digitizing. In addition, reconciling the differences in floorplans from various renovation projects required consulting with the client and taking physical measurements (see section 5.3).

\subsubsection{Analysis of Uploading to ArcGIS Online}

Ensuring all the data uploaded correctly to ArcGIS Online proved more challenging than expected. Initially, uploading the indoor layers separately from the FM layers caused Field Maps to not recognize the ArcGIS Indoors attributes, resulting in the floor filter not functioning. It would not have been ideal to upload a separate copy of the indoor layers 
for each Web Map as any edits, changes, or additions made in the future would have to be copied to each. This issue was later pinpointed to a difference in how ArcGIS Online was storing the layer titles, not an issue with the data themselves. The titles were manually changed, and Field Maps continued to function as expected.

On several occasions, uploading failed without providing a useful error code. Through carefully backtracking changes made since the last successful upload, the issue was found to be caused by two attribute domains assigned to long integer fields. The domains were removed and manually re-added in ArcGIS Online. It's possible that the coded values were interfering with attribute rules because new domains functioned fine in ArcGIS Online.

While Esri's default basemaps use Web Mercator, it is possible to author Web Maps in any chosen spatial reference using a custom basemap. It was attempted to create a basemap of the outdoor features using SPCS CA Zone 5. This would make measurements more accurate and would mean that ArcGIS Online would not have to perform on-the-fly projections for the layers. However, the tiling scheme for SPCS used to render the basemap at multiple scales can't create tiles at such a large scale. Therefore, Web Mercator was ultimately used.

\subsubsection{Analysis of System Design}

Creating the inventory and assets systems presented significant design challenges. It was initially thought that Field Maps would not support related tables, making the current implementation of the inventory system infeasible for mobile. This functionality, however, was a relatively recent addition to Field Maps and was not widely documented, but the system does now function as originally intended. The assets system was derived from the reserve study, which conflated multiple buildings into single values, essentially adding the values together. These values were separated in Excel and calculated appropriately so that points could be created for each building (see section 5.3.6).

It was also a challenge to work out how to spatially represent the asset items. Roofing material, for instance, would be best represented as polygons, whereas a water heater would be best as a point. For the system ease-of-use, however, these were all represented as point features (see section 5.3.6). Input was not provided by the client on where these features should be located, so the locations were chosen by best approximation. If the client wishes, they can move these features to more suitable locations in the future. For features intended to cover an entire building, like roofing materials, an additional level called "roof" was added and is selectable in the system's filters (figure 6-3, 6-5).

It was intended for attribute rules to be carried over from the geodatabase and to add contingent values. Attribute rules would be used to auto-populate Indoors features while contingent values would filter available inputs based on previous selections. Therefore, when populating indoors attributes, selecting a facility ID would filter the available values for level ID and unit ID to only those within the facility and would automatically populate the respective names. Neither of these features are currently supported in ArcGIS Online, but are apparently - at least in Field Maps - due to arrive by the end of 2021 (Shaner, 2021). 


\subsubsection{Analysis of System Construction}

Constructing the system went relatively smoothly after the data were in ArcGIS Online, although some problems were encountered. Issues in system construction were underscored by compatibility issues between the newly released Map Viewer and Map Viewer Classic. Field Maps does not fully support the pop-up features of the new Map Viewer, so it is recommended that maps for Field Maps use Map Viewer Classic if custom popups are desired (Clifford, 2021). However, certain functionality in Dashboards requires the use of the new Map Viewer, including the ability to format legends. If care is taken, it is possible to work with the same map in both Map Viewer versions, but in this case any customizations made to the pop-up in Map Viewer Classic were thrown away the next time the map was saved in the new Map Viewer. Popups, therefore, were only configured in Map Viewer Classic after all other map settings were set in Map Viewer. According to Clifford, it is hopeful that compatibility will be added in the coming months (as of November 2021), but users should be aware of this when using Map Viewer in the future.

Another issue in system construction was feature rendering in Map Viewer, and by extension the Web Apps and Dashboards. Features on the map are over-simplified, showing vertices in their wrong locations or with generalized vertices, resulting in jagged and misshapen edges (figure 7-2). Visually, this issue is most noticeable in the Details layer. In searching for a solution, it was determined that this is probably an issue with the generalization algorithm used by Map Viewer. With the map set to the largest scale possible, which in this case is 1:35, the features appear relatively correct. Unfortunately, this does not appear to be a problem that can be fixed without an update to Map Viewer. An alternative would be to publish the Indoors layers as tile, which would store tiles at set intervals rather than rendering them dynamically. However, this would break the ArcGIS Indoors functionality. 

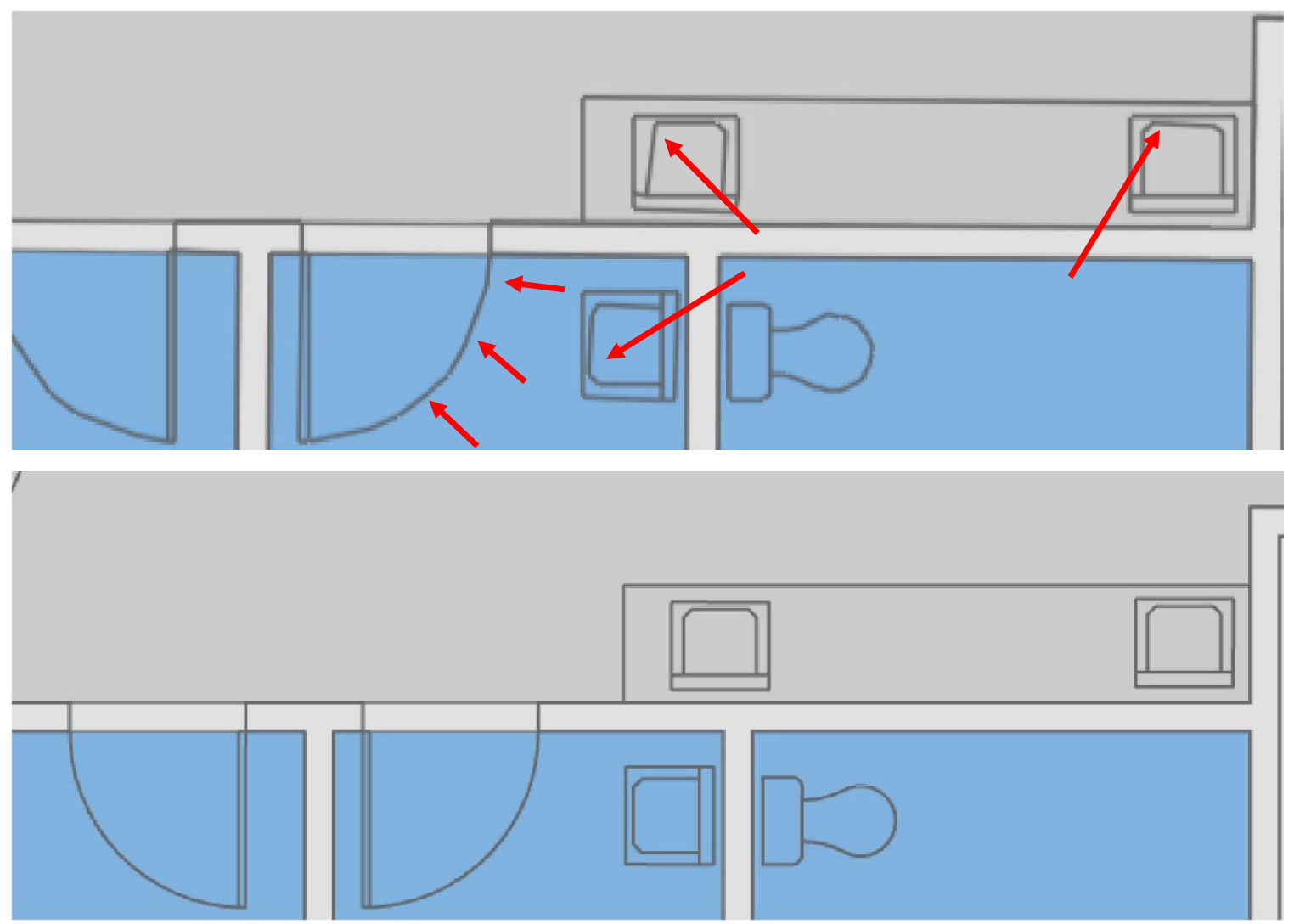

Figure 7-2: Rendering in ArcGIS Online (top) vs. ArcGIS Pro (bottom)

\subsection{Summary}

Implementation resulted in a system which successfully runs in a web browser or mobile device and meets the specified needs of the client, although certain issues and errors were encountered throughout. Georeferencing presented challenges in how ArcGIS Pro stored GCPs. Most of the digitizing time was taken reconciling differences between floorplans, correcting mistakes, and manually taking measurements. Uploading to ArcGIS Online was challenged by differences in how Online interprets attribute data. System design and construction was similarly hindered by ArcGIS Online compatibility issues, resulting in changes to workflow and overall design. 


\section{Chapter 8 - Conclusions and Future Work}

Montessori in Redlands (MIR) is in a city with a strong propensity for GIS. The largest GIS company, Esri, is headquartered there. The University of Redlands (UoR), from which the author of this paper graduated, is often dubbed "a spatial university." MIR itself has a student body largely comprising the children of Esri employees. Recognizing the potential GIS has as a solution to far-reaching problems, MIR staff turned to the UoR Master of Science in GIS students to develop a system that would fit their needs. This project offers a facilities management solution to address these needs, creating userfriendly interfaces with the flexibility for future expansion.

\subsection{Conclusions}

MIR requested a solution to the following three problems:

- Inability to track classroom inventory

- Lack of maintenance and replacement for permanent assets

- Nonexistence of high-quality campus maps for emergency first responders

The system created by this project meets all three of these requirements and provides room for future expansion. The indoor campus map can now serve as a canvas as more ideas for indoor mapping, and indeed outdoor mapping, are implemented, some of which are described in section 8.2.

\subsubsection{Inventory Tracking}

The inventory problem was solved by creating a feature class of catalog points, or inventory points for each room and outdoor location, such as sheds or playground areas. These points are related to a standalone table that lists the items for each location and are referenced using a location ID. This was an important problem for MIR as the school often does not know what items they have in their possession or where they are located. Additionally, certain items in classrooms are important to the Montessori teaching method and are thoughtfully chosen by teachers for each age group and for the unique qualities of every group of students. It is important that these can be accurately located. Cited in the initial meetings was an example of teachers ordering new items - a shelf, for instance - and receiving them only to find a suitable item was already in storage. Now, users can simply tap on a room or search by name to instantly find what they are looking for (see sections 6.1.3 and 5.3.6).

\subsubsection{Permanent Asset Management}

Managing permanent assets was solved by developing a Dashboard and a Web App to visualize the permanent assets found throughout the campus buildings. Permanent assets include non-movable items such as buildings' roofing, hot water heaters, and lighting fixtures. The Dashboard provides descriptive statistics of those assets and allows users to query and find assets of a specific type, in a specific location, or of a specific urgency. For this problem, MIR expressed concern that they were not allocating enough resources for maintaining and replacing these assets. As such, the goal of this system component is 
not to dictate when assets need to be replaced, but to provide a snapshot of what the school needs to be prepared for (see sections 6.1 and 5.3.6).

\subsubsection{Emergency First Responder Map}

The only map first responders had access to was a hand-drawn campus map, which included basic outdoor features, some indoor features, and door numbers. This system creates an interactive map with the goal of quickly allowing first responders to locate areas on campus. Larger rooms, such as classrooms and multipurpose rooms, are labeled with easy-to-read text. Upon opening the Dashboard, users can jump to buildings, filter floors, and flash rooms with three clicks. In Field Maps, users can jump to buildings using pre-defined bookmarks for each building and locate rooms based on the labeling (see sections 6.1.4, 6.2, 5.3.6).

\subsection{Future Work}

The facilities management system described in this project addresses the problems MIR identified as high concern. This section describes how the system could be expanded in the future.

\subsubsection{Expanding ArcGIS Indoors Functionality}

This system is a relatively rudimentary deployment of the ArcGIS Indoors data model. ArcGIS Indoors is available as a separately licensed product that takes advantage of the Indoors data model, which this project does not utilize. A license provides access to expanded functionality in ArcGIS Pro, including geoprocessing tools to automatically prepare Indoors feature classes. Licensed organizations can also use the Indoors Viewer web application and the ArcGIS Indoors mobile application. These provide the ability to navigate indoors, explore campus areas, reserve workspaces, and integrate calendars to find meeting locations.

Advanced features such as indoor navigation require additional feature classes to function properly, including Points Of Interest and the Indoors network. Points of Interest contains points to relevant locations on the campus. The Indoors network contains all the possible pathways between areas, considering obstructions and floor transitions such as elevators. ArcGIS Indoors can also take advantage of Web Scenes to display buildings in 3-D. This is helpful in providing a better overview of a campus and can be useful in indoor navigation where traversing floors is important. In the future, an analyst could implement these functions to enable the full functionality of ArcGIS Indoors.

\subsubsection{Creating Physical Plant Layers}

The indoors map created for this project mainly includes the architectural features of buildings, or those features representing things most people interact with on a day-to-day basis. However, GIS is widely used for understanding the physical plant infrastructure of a campus. Plumbing, ductwork, and sprinkler systems, for instance, are all included in the scanned documents. These could be digitized as their own layers and used by the

facilities coordinator and maintenance staff to locate and maintain the physical features of 
a campus. As with the architectural features, however, they are likely not entirely accurate and would probably require extensive physical verification. Sprinkler systems, for example, mainly exist underground. Therefore, when leaks happen, it is not always immediately evident where they are occurring. A sprinkler system map layer would allow staff members to immediately locate the placement of the underground piping, hastening the search for the leak. Locating these features today would require searching through the floorplans, assuming they are accurate. Digitizing these features would require a significant amount of work and the system would need to be modified to accommodate underground layers.

\subsubsection{Expanding Safety Layers}

Currently, the Safety map only contains door numbers with labels. This could be expanded in the future to include, for example, evacuation routes, evacuation zones, fire extinguishers, fire alarm pull switches, fire hydrants, defibrillators, and first aid kits. Such layers could be made floor-aware by attributing for ArcGIS Indoors. This would be a relatively simple task for an MIR staff member to do without ArcGIS Pro as Field Maps or Map Viewer in ArcGIS online can be used to create layers and collect points.

\subsubsection{Tree Mapping}

As part of a previous project, Wendy Wallace (2010) created a point layer of the trees on campus with the help of MIR students. These points are more accurate than those in the current basemap, which were digitized from satellite imagery and include attributes such as age and species. However, because the data are more than a decade out of date they were ultimately not included in the project. Future work here would include updating the features and their attributes, including deleting or collecting new points as necessary. Unlike in 2010, the GPS found in most smartphones, with the assistance of correction, would be adequate for a project such as this. The trees are major financial and aesthetic assets to MIR. Preserving them, including its expansive grapefruit grove, is important, especially as organizations everywhere grapple with the consequences of a changing planet.

\subsection{Summary}

This project addressed facilities management and security concerns identified by Montessori in Redlands through the implementation of an online GIS system. Userfriendly and fully accessible online, the system satisfies the requirements as outlined in preliminary meetings. In the future, more work could be done to expand the system and improve functionality. Included in future work is deploying a full ArcGIS Indoors license, creating physical plant layers, expanding safety layers, and updating the 2010 tree map. 


\section{Works Cited}

Agrawal, S., \& Gupta, R. (2017). Web GIS and its architecture: A review. Arabian Journal of Geosciences, 10(23), 1-13.

Bahri, M. A. S., Abdul Maulud, K. N., Rahman, M. A., Ridzuan Oon, A. O., Che Ani, A. I., Che Hashim, C. H., Karim, H., Hasbullah, M. S., \& Aziz, M. Z. (2019).

Development of GIS Database and Facility Management System: Asset and Space in UKM. ISPRS - International Archives of the Photogrammetry, Remote Sensing and Spatial Information Sciences, XLII-4/W16, 563-571.

https://doi.org/10.5194/isprs-archives-XLII-4-W16-563-2019

Baker, T. R. (2015). WebGIS in Education. In O. Muñiz Solari, A. Demirci, \& J. Schee (Eds.), Geospatial Technologies and Geography Education in a Changing World: Geospatial Practices and Lessons Learned (pp. 105-115). Springer Japan. https://doi.org/10.1007/978-4-431-55519-3_9

Beauregard, M. A., \& Ayer, S. K. (2018). Maintaining performance: Understanding the relationship between facility management and academic performance at K-12 schools in the State of Arizona. Facilities, 36(11/12), 618-634. ABI/INFORM Collection. https://doi.org/10.1108/F-11-2017-0111

Bingaith, A. (2010). Implementing GIS for Facilities Management at the California Institute of Technology [Master's Thesis, University of Redlands]. https://doi.org/10.26716/redlands/master/2010.2

Bolstad, P. (2019). GIS Fundamentals: A First Text on Geographic Information Systems (Sixth edition). XanEdu Publishing Inc.

Bröchner, J., Haugen, T., \& Lindkvist, C. (2019). Shaping tomorrow's facilities management. Facilities, 37(7/8), 366-380. ABI/INFORM Collection. https://doi.org/10.1108/F-10-2018-0126

Bruner, J. S. (1999). The process of education. Harvard University Press.

Clifford, J. (2021, November 18). Field Maps and Map Viewer. ArcGIS Blog. https://www.esri.com/arcgis-blog/products/field-maps/field-mobility/field-mapsand-map-viewer/

de Jong, M., Triantafyllou, G., Spinoza Andreo, G., Dardavesis, I., Kumar, P., \& Maundri Prihanggo, M. (2021). Building Rhythms: Reopening the workspace with indoor localisation [Master's Thesis]. Delft University of Technology.

Dodge, S., Xu, J., \& Stenger, B. (2017). Parsing floor plan images. 2017 Fifteenth IAPR International Conference on Machine Vision Applications (MVA), 358-361. https://doi.org/10.23919/MVA.2017.7986875 
Drion, B., Melissen, F., \& Wood, R. (2012). Facilities management: Lost, or regained? Facilities, 30(5/6), 254-261. ABI/INFORM Collection. https://doi.org/10.1108/02632771211208512

Duffy, F. (2000). Design and facilities management in a time of change. Facilities, 18(10/11/12), 371. ABI/INFORM Collection. https://doi.org/10.1108/02632770010349592

Duyar, I. (2010). Relationship between school facility conditions and the delivery of instruction: Evidence from a national survey of school principals. Journal of Facilities Management, 8(1), 8-25. ABI/INFORM Collection. https://doi.org/10.1108/14725961011019058

El-Gamily, H. I., \& Al-Rasheed, K. (2015). Deploying an Interactive GIS System for Facility and Asset Management: Case Study-Ministry of Education, Kuwait. In Journal of Geographic Information System (Vol. 7, Issue 2, p. 11).

Ellis, G. (2021, September 1). What Are As Built Drawings? Autodesk Construction Cloud. https://constructionblog.autodesk.com/as-built-drawings/

Esri. (n.d.-a). About georeferencing CAD data-ArcGIS Pro|Documentation. ESRI. Retrieved March 21, 2021, from https://pro.arcgis.com/en/proapp/latest/help/data/cad/about-georeferencing-cad-data.htm

Esri. (n.d.-b). ArcGIS Indoors Information Model-ArcGIS Pro | Documentation. ESRI. Retrieved March 22, 2021, from https://pro.arcgis.com/en/proapp/latest/help/data/indoors/arcgis-indoors-information-model.htm

Esri. (n.d.-c). FloorFilter widget | Sample Code | ArcGIS API for JavaScript $4.21 \mid$ ArcGIS Developer. ArcGIS API for JavaScript 4.21. Retrieved November 22, 2021, from https://developers.arcgis.com/javascript/latest/sample-code/widgetsfloorfilter/

Esri. (n.d.-d). Overview of georeferencing-ArcGIS Pro|Documentation. ESRI. Retrieved October 4, 2021, from https://pro.arcgis.com/en/proapp/latest/help/data/imagery/overview-of-georeferencing.htm

Esri. (n.d.-e). Problem: A generic error message is returned when configuring ArcGIS Web Adaptor for ArcGIS Server. Esri. Retrieved November 22, 2021, from https://support.esri.com/en/technical-article/000024787

Esri. (n.d.-f). Raster file formats-ArcGIS Pro| Documentation. Esri. Retrieved November 16, 2021, from https://pro.arcgis.com/en/proapp/latest/help/data/imagery/supported-raster-dataset-file-formats.htm

Esri. (n.d.-g). What is ArcScan?-ArcMap | Documentation. ESRI. Retrieved October 4, 2021, from https://desktop.arcgis.com/en/arcmap/latest/extensions/arcscan/whatis-arcscan-.htm 
Forsberg, K., Mooz, H., \& Cotterman, H. (2005). Visualizing Project Management: Models and Frameworks for Mastering Complex Systems. John Wiley \& Sons.

Howard, D. L. (2002). Management Speak in facilities management. Critical Quarterly, 44(4), 25-31. https://doi.org/10.1111/1467-8705.00451

IFMA. (2021a). History. International Facilities Management Association. https://www.ifma.org/about/about-ifma/history/

IFMA. (2021b). What is Facility Management. International Facilities Management Association. https://www.ifma.org/about/what-is-facility-management/\#a1

Kamaruzzaman, S. N., \& Zawawi, E. M. A. (2010). Development of facilities management in Malaysia. Journal of Facilities Management, 8(1), 75-81. ABI/INFORM Collection. https://doi.org/10.1108/14725961011019094

Kowalski, T. J. (2002). Planning and managing school facilities. Greenwood Publishing Group.

Montessori In Redlands. (2020, December 22). About MIR. Montessori in Redlands. https://www.mir.org/about-mir

Montessori, M. M. Jr. (1976). Education for Human Development: Understanding Montessori. Schocken.

Morgan, L. (2001). Facility Conditions and Student Test Performance in the Milwaukee Public Schools (Evaluative No. ED459593). Council of Educational Facility Planners, International.

Morgan, M. F. (2009). CAD-GIS interoperability issues for facilities management: Enabling inter-disciplinary workflows [Master's Thesis, University of South Carolina]. In ProQuest Dissertations and Theses (304995579). Publicly Available Content Database.

https://ezproxy.redlands.edu/login?url=https://www.proquest.com/dissertationstheses/cad-gis-interoperability-issues-facilities/docview/304995579/se2 ? accountid $=14729$

Mwaniki, M. W., \& Odera, P. A. (2014). Application of GIS in Facility Space Management: A Case Study of ILRI. International Journal of Science and Research, 3(9).

Shaner, J. (2021, January 3). Introducing smart forms in ArcGIS Field Maps. ArcGIS Blog. https://www.esri.com/arcgis-blog/products/field-maps/fieldmobility/introducing-arcgis-smart-forms/

Stalcup, K. (2021, May 17). AWS vs Azure vs Google Cloud Market Share 2021: What the Latest Data Shows. ParkMyCloud. https://www.parkmycloud.com/blog/awsvs-azure-vs-google-cloud-market-share/www.parkmycloud.com 
Tay, L., \& Ooi, J. T. L. (2001). Facilities management: A "Jack of all trades"? Facilities, 19(10), 357-362. ABI/INFORM Collection. https://doi.org/10.1108/EUM0000000005534

Tekavec, J., \& Lisec, A. (2020). Cadastral data as a source for 3D indoor modelling. 3D Land Administration for 3D Land Uses, 98, 104322. https://doi.org/10.1016/j.landusepol.2019.104322

Wallace, W. L. (2010). Montessori Green School Initiative Mapping Project [Master's Thesis, University of Redlands]. https://doi.org/10.26716/redlands/master/2010.21. https://inspire.redlands.edu/gis_gradproj/166/

Wittreich, W. P. (1997). Beyond CAD Into GIS: A Look at Autodesk World 1.0, AutoCAD Map 2.0, and Autodesk MapGuide. Cadence, 12, 66.

Wu, Y., Shang, J., Chen, P., Zlatanova, S., Hu, X., \& Zhou, Z. (2021). Indoor mapping and modeling by parsing floor plan images. International Journal of Geographical Information Science, 35(6), 1205-1231. 


\title{
Appendix A. Schema Report
}

\section{Geodatabase schema diagram}

This diagram was auto-generated by the Geodatabase Diagrammer application sample and contains graphic elements that you can use to produce a data model diagram. This sample is available from the ArcScripts site on www.esri.com. You can find examples of finished data model diagrams at the data model section of arconline.esri.com.

\author{
Geodatabase D: \OneDrive - University of Redlands \MIP\MIR_FM\MIR_FM.gdb
}

Date generated Sunday, November 28, 2021

\section{Domains}

\begin{tabular}{|c|c|}
\hline $\begin{array}{l}\text { Coded value domain } \\
\text { Level_name_long } \\
\text { Description Long name for level } \\
\text { Field type String } \\
\text { Split policy Default value } \\
\text { Merge policy Default value }\end{array}$ & \\
\hline Code & Description \\
\hline Level 1 & Level 1 \\
\hline Level 2 & Level 2 \\
\hline NO_VALUE & No Value \\
\hline Roof & Roof \\
\hline
\end{tabular}

\begin{tabular}{|c|c|}
\hline \multicolumn{2}{|l|}{$\begin{array}{l}\text { Coded value domain } \\
\text { Facility_name }\end{array}$} \\
\hline $\begin{aligned} \text { Description } & \text { Facility Name } \\
\text { Field type } & \text { String } \\
\text { Split policy } & \text { Default value } \\
\text { Merge policy } & \text { Default value }\end{aligned}$ & \\
\hline Code & Description \\
\hline Building $A$ & Building $A$ \\
\hline Build ing B & Building $B$ \\
\hline Build ing $C$ & Build ing C \\
\hline Building D & Build ing $D$ \\
\hline Building $E$ & Building $\mathrm{E}$ \\
\hline Build ing $M$ & Building $M$ \\
\hline NO_VALUE & No Value \\
\hline
\end{tabular}




\begin{tabular}{|cc|}
\hline Coded value domain & \\
Unit_Use & \\
Description Units use type & \\
Field type String & \\
Split policy Default value & \\
Merge policy Default value & \\
Code & Description \\
\hline Office & Office \\
Hallway & Hallway \\
Storage & Storage \\
Multiurpose & Multipurpose Room \\
Lunch & Lunch Room \\
Restroom & Restroom \\
Stairway & Stairway \\
Electrical & Electrical \\
Community & Community (Classroom) \\
Kitchen & Kitchen \\
Workroom & Workroom \\
Laundry & Laundry \\
Lobby & Lobby \\
NO_VALUE & No Value \\
\hline
\end{tabular}

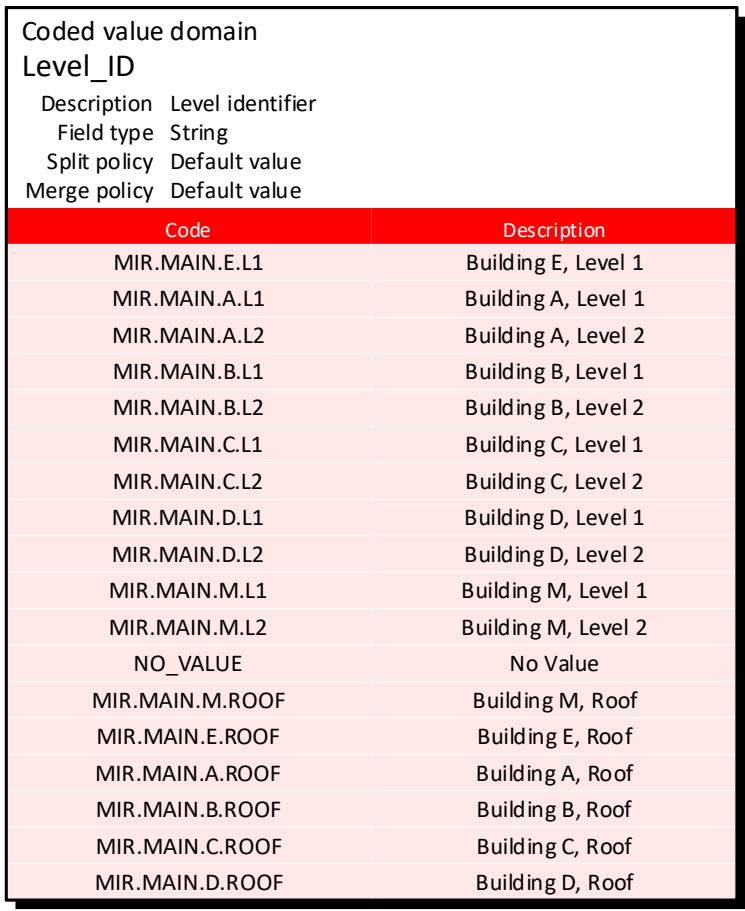

Coded value domain

Site_ID

Description Site identifier

Field type String

Split policy Default value

Merge policy Default value

\begin{tabular}{|cc|}
\hline Code & Description \\
\hline MIR.MAIN & MIR Main Campus \\
NO_VALUE & No Value \\
\hline
\end{tabular}




\begin{tabular}{|c|c|}
\hline $\begin{array}{l}\text { Coded value domain } \\
\text { Facility } \\
\text { Description Facility identifier } \\
\text { Field type String } \\
\text { Split policy Default value } \\
\text { Merge policy Default value }\end{array}$ & \\
\hline Code & Description \\
\hline MIR.MAIN.A & Build ing $\mathrm{A}$ \\
\hline MIR.MAIN.B & Build ing $B$ \\
\hline MIR.MAIN.C & Build ing $C$ \\
\hline MIR.MAIN.D & Building D \\
\hline MIR.MAIN.E & Build ing $\mathrm{E}$ \\
\hline MIR.MAIN.M & Build ing $\mathrm{M}$ \\
\hline NO_VALUE & No Value \\
\hline
\end{tabular}

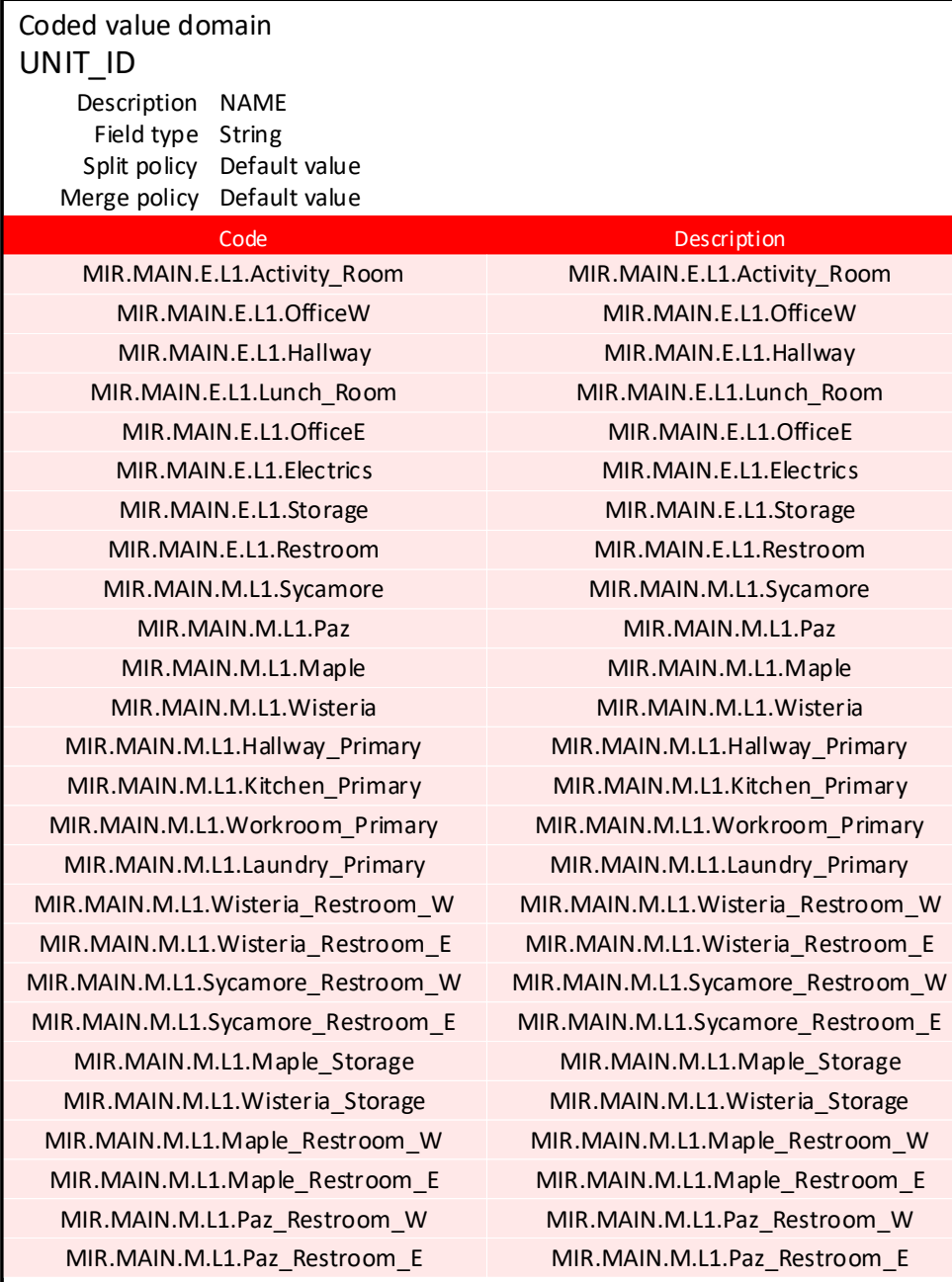

Further values omitted due to length 


\begin{tabular}{|cc|}
\hline Coded value domain \\
Level_name_short \\
Description Short name for level \\
Field type String \\
Split policy Default value \\
Merge policy Default value \\
\hline Code \\
\hline L1 \\
L2 \\
NO_VALUE & Description \\
Roof & L1 \\
\hline
\end{tabular}

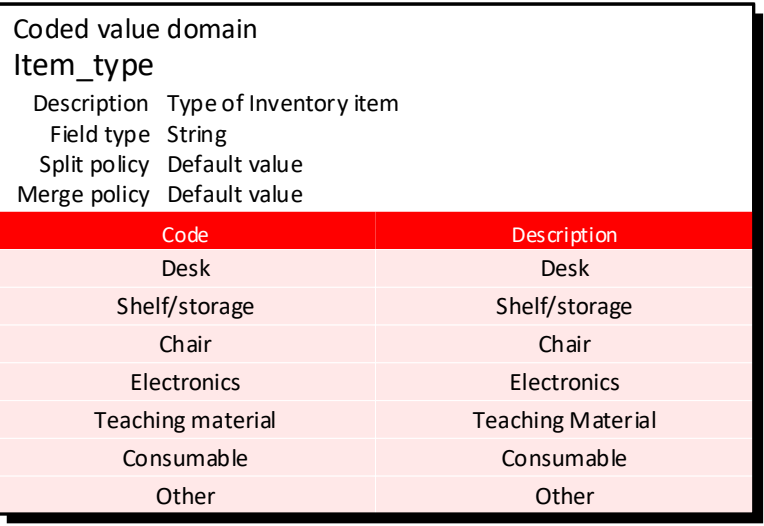

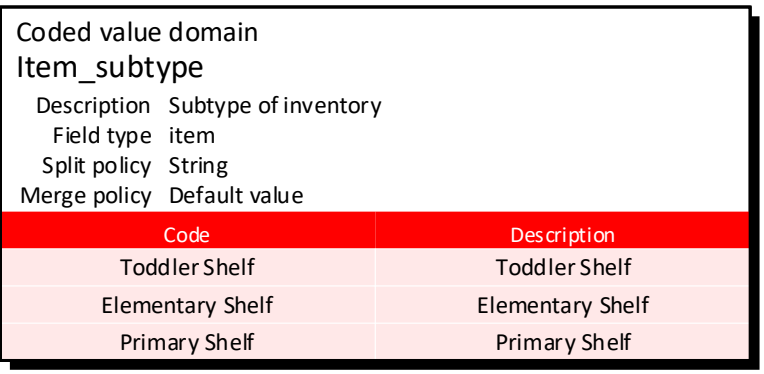

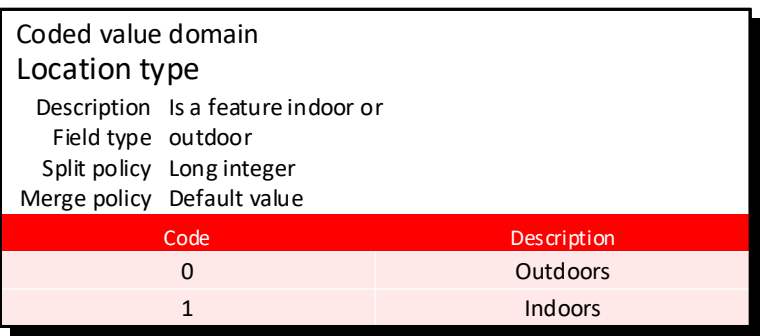




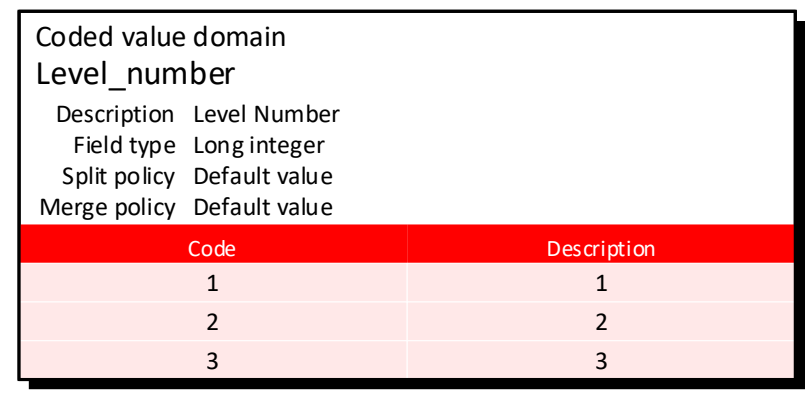

Coded value domain

Details_Use

Description Details use type

Field type String

Split policy Default value

Merge policy Default value

\begin{tabular}{|c|c|}
\hline Code & Description \\
\hline A-WALL-EXTR & Exterior wall, interior line \\
A-WALL-INFR & Exterior wall, exterior line \\
A-WALL & Interior wall \\
A-WALL-PRHT & Interior Wall, partial height \\
A-AREA-BDRY & Area Boundary \\
A-COLS & Comumn \\
A-AREA-LINE & Area Line \\
A-DOOR & Door \\
A-WALL-GLAS & Glass \\
A-FLOR-STRS & Stairs \\
A-ANNO-TEXT & Annotation \\
NO_VALUE & No Value \\
P-FIXT & Plumbing Fixture \\
A-FLOR-APPL & Appliance \\
A-FLOR-TPTN & Toilet Partition \\
A-FLOR-WDWK & Cabinet or Counter \\
A-FURN-PNLS & Cubicle Partition \\
A-FURN-WKSF & Cubicle Work Surface \\
\hline
\end{tabular}

\section{Feature Classes}

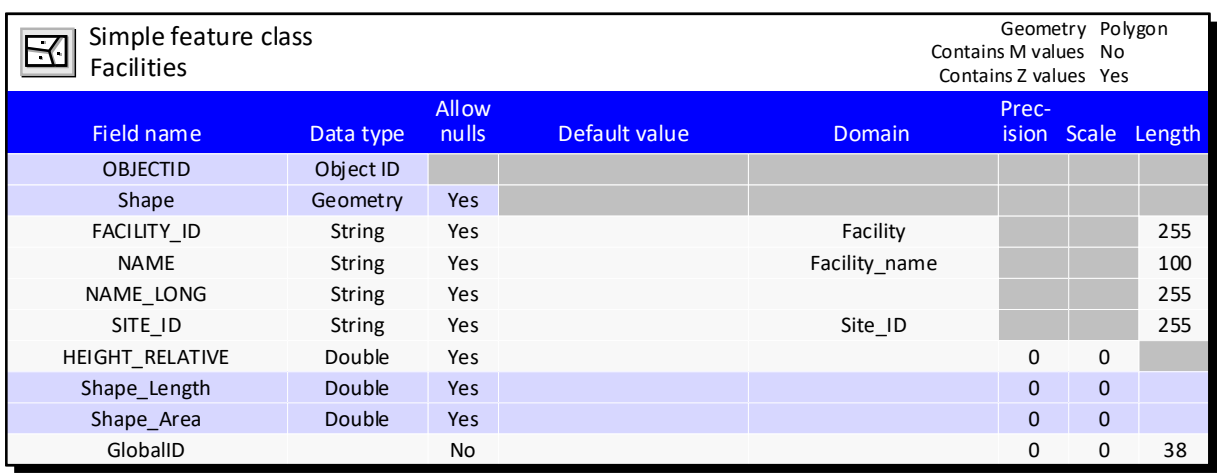




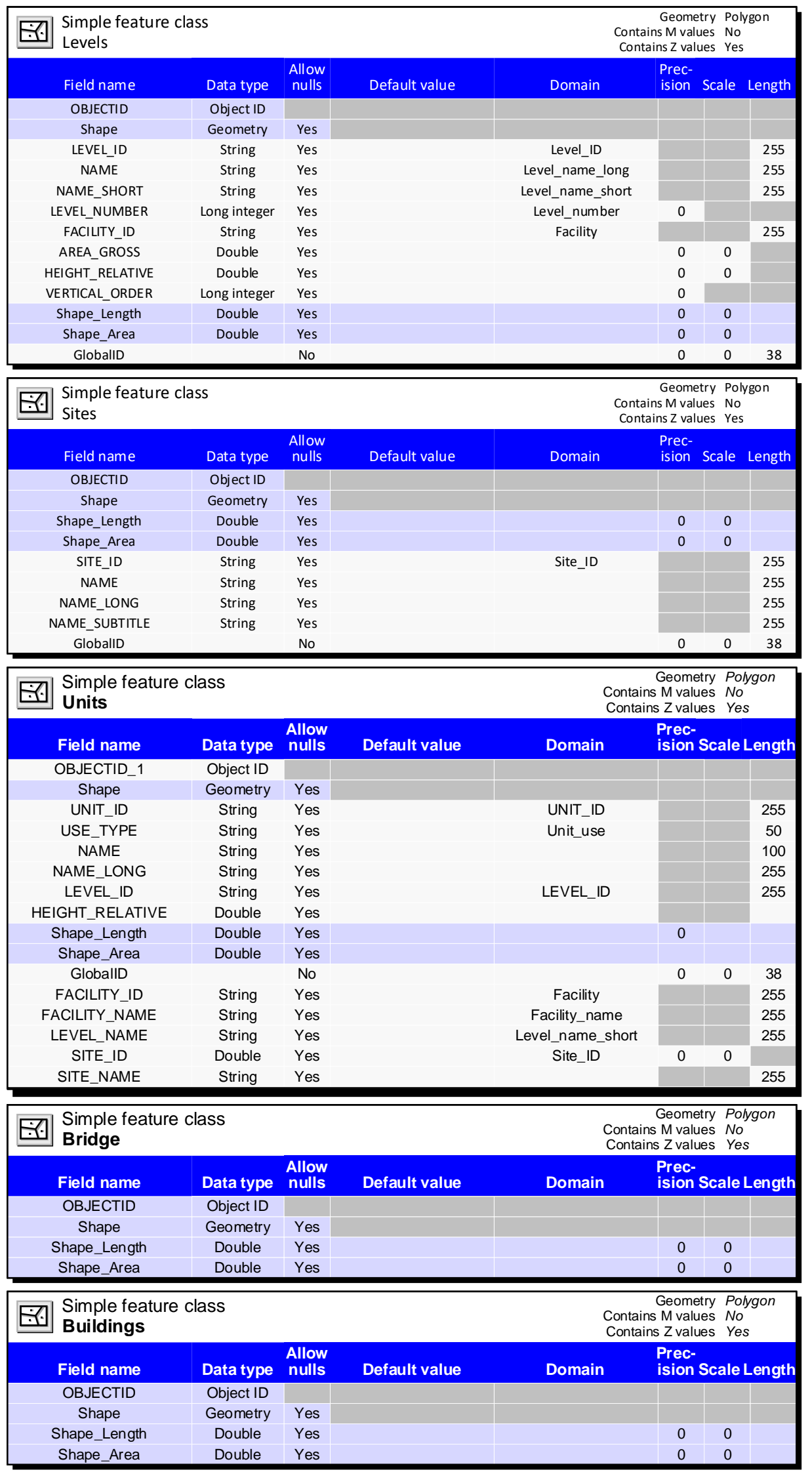




\begin{tabular}{|c|c|c|c|c|c|c|c|}
\hline \multicolumn{4}{|c|}{$\begin{array}{ll}\text { Simple feature class } \\
\text { Court }\end{array}$} & \multicolumn{4}{|c|}{$\begin{aligned} \text { Geometry } & \text { Polygon } \\
\text { Contains M values } & N o \\
\text { Contains } Z \text { values } & \text { Yes }\end{aligned}$} \\
\hline Field name & Data type & $\begin{array}{l}\text { Allow } \\
\text { nulls }\end{array}$ & Default value & \multirow[t]{2}{*}{ Domain } & \multicolumn{3}{|c|}{$\begin{array}{l}\text { Prec- } \\
\text { ision Scale Length }\end{array}$} \\
\hline OBJECTID & Object ID & & & & & & \\
\hline Shape & Geometry & Yes & & & & & \\
\hline Shape_Length & Double & Yes & & & 0 & 0 & \\
\hline Shape_Area & Double & Yes & & & 0 & 0 & \\
\hline
\end{tabular}

\begin{tabular}{|c|c|c|c|c|c|c|c|}
\hline \multicolumn{5}{|c|}{\begin{tabular}{l|l} 
Simple feature class \\
Creek
\end{tabular}} & $\begin{array}{l}\text { Geom } \\
\text { s M val } \\
\text { is } Z \text { val }\end{array}$ & & $\begin{array}{l}b_{\text {lygon }} \\
o \\
\text { es }\end{array}$ \\
\hline Field name & Data type & $\begin{array}{l}\text { Allow } \\
\text { nulls }\end{array}$ & Default value & Domain & \multicolumn{3}{|c|}{$\begin{array}{l}\text { Prec- } \\
\text { ision Scale Length }\end{array}$} \\
\hline OBJECTID & Object ID & & & & & & \\
\hline Shape & Geometry & Yes & & & & & \\
\hline Shape_Length & Double & Yes & & & 0 & 0 & \\
\hline Shape_Area & Double & Yes & & & 0 & 0 & \\
\hline
\end{tabular}

\begin{tabular}{|c|c|c|c|c|c|c|c|}
\hline \&:- Simple feat & lass & & & \multicolumn{4}{|c|}{$\begin{aligned} \text { Geometry } & \text { Polygon } \\
\text { Contains M values } & N o \\
\text { Contains } Z \text { values } & \text { Yes }\end{aligned}$} \\
\hline Field name & Data type & $\begin{array}{l}\text { Allow } \\
\text { nulls }\end{array}$ & Default value & Domain & $\begin{array}{l}\text { Prec- } \\
\text { ision } \mathrm{s}\end{array}$ & & \\
\hline OBJECTID & Object ID & & & & & & \\
\hline Shape & Geometry & Yes & & & & & \\
\hline Shape_Length & Double & Yes & & & 0 & 0 & \\
\hline Shape_Area & Double & Yes & & & 0 & 0 & \\
\hline
\end{tabular}

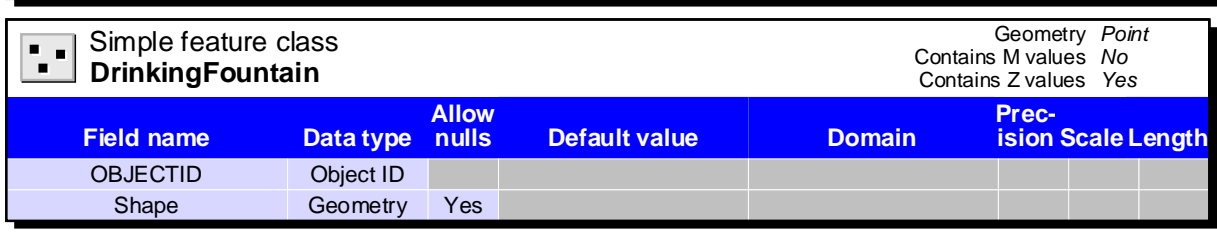

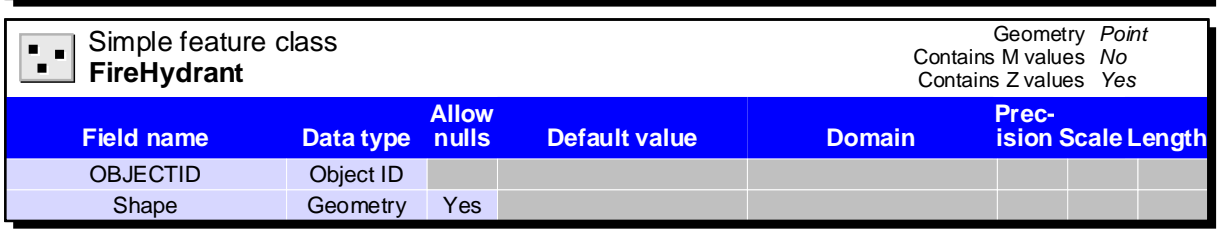

\begin{tabular}{|c|c|c|c|c|c|c|c|}
\hline $\begin{array}{ll}\text { Simple fea } \\
\text { Lawn }\end{array}$ & ass & & & & $\begin{array}{l}\text { Geome } \\
\text { ns M valu } \\
\text { ins } Z \text { valu }\end{array}$ & $\begin{array}{l}\text { ry } P o \\
\text { es No } \\
\text { es } Y e\end{array}$ & $\begin{array}{l}\text { olygon } \\
o \text { os }\end{array}$ \\
\hline Field name & Data type & $\begin{array}{l}\text { Allow } \\
\text { nulls }\end{array}$ & Default value & Domain & $\begin{array}{l}\text { Prec- } \\
\text { ision }\end{array}$ & & Length \\
\hline OBJECTID & Object ID & & & & & & \\
\hline Shape & Geometry & Yes & & & & & \\
\hline Shape_Length & Double & Yes & & & 0 & 0 & \\
\hline Shape_Area & Double & Yes & & & 0 & 0 & \\
\hline
\end{tabular}

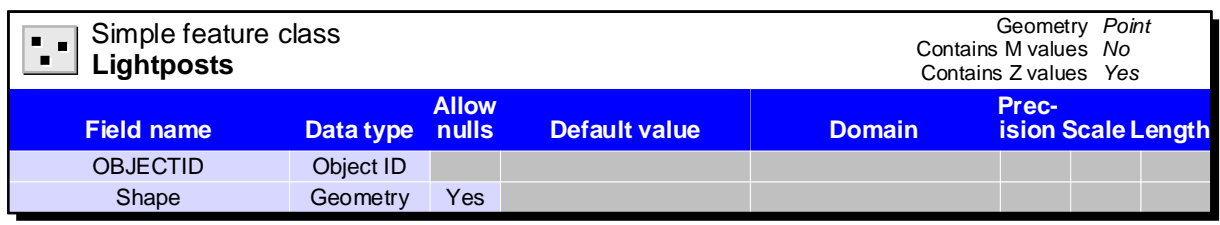

\begin{tabular}{|c|c|c|c|c|c|c|c|}
\hline \multicolumn{4}{|c|}{$\begin{array}{ll}\text { Simple feature class } \\
\text { Other }\end{array}$} & \multicolumn{4}{|c|}{$\begin{aligned} \text { Geometry } & \text { Polygon } \\
\text { Contains M values } & N o \\
\text { Contains Z values } & \text { Yes }\end{aligned}$} \\
\hline Field name & Data type & $\begin{array}{l}\text { Allow } \\
\text { nulls }\end{array}$ & Default value & Domain & $\begin{array}{l}\text { Prec- } \\
\text { ision }\end{array}$ & & Length \\
\hline OBJECTID & Object ID & & & & & & \\
\hline Shape & Geometry & Yes & & & & & \\
\hline Shape_Length & Double & Yes & & & 0 & 0 & \\
\hline Shape_Area & Double & Yes & & & 0 & 0 & \\
\hline
\end{tabular}




\begin{tabular}{|c|c|c|c|c|c|c|c|}
\hline $\begin{array}{ll}\text { Simple feat } \\
\text { Parking_Lc }\end{array}$ & & & & & $\begin{array}{r}\text { Geomet } \\
\text { Contains M valu } \\
\text { Contains } Z \text { value }\end{array}$ & $\begin{array}{ll}\text { ry } & P o \\
\text { ss } & N o \\
\text { s } & Y e .\end{array}$ & $\begin{array}{l}\text { blygon } \\
b_{\text {es }}\end{array}$ \\
\hline Field name & Data type & $\begin{array}{l}\text { Allow } \\
\text { nulls }\end{array}$ & Default value & Domain & $\begin{array}{l}\text { Prec- } \\
\text { ision }\end{array}$ & icale & Length \\
\hline OBJECTID & Object ID & & & & & & \\
\hline Shape & Geometry & Yes & & & & & \\
\hline Shape_Length & Double & Yes & & & 0 & 0 & \\
\hline Shape_Area & Double & Yes & & & 0 & 0 & \\
\hline \begin{tabular}{|l|l|} 
I Simple feat \\
Parking_S
\end{tabular} & & & & & $\begin{array}{r}\text { Geomet } \\
\text { Contains M valu } \\
\text { Contains } Z \text { value }\end{array}$ & 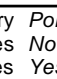 & $\begin{array}{l}\text { blyline } \\
0 \\
\text { ss }\end{array}$ \\
\hline Field name & Data type & $\begin{array}{l}\text { Allow } \\
\text { nulls }\end{array}$ & Default value & Domain & $\begin{array}{l}\text { Prec- } \\
\text { ision } 5\end{array}$ & icale & Length \\
\hline OBJECTID & Object ID & & & & & & \\
\hline Shape & Geometry & Yes & & & & & \\
\hline Shape_Length & Double & Yes & & & 0 & 0 & \\
\hline \begin{tabular}{|l|l|} 
S Simple feat \\
Pathway
\end{tabular} & lass & & & & $\begin{array}{r}\text { Geomet } \\
\text { Contains M value } \\
\text { Contains } Z \text { value }\end{array}$ & 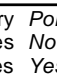 & $\begin{array}{l}\text { Dlyline } \\
0 \\
\text { es }\end{array}$ \\
\hline Field name & Data type & $\begin{array}{l}\text { Allow } \\
\text { nulls }\end{array}$ & Default value & Domain & $\begin{array}{l}\text { Prec- } \\
\text { ision }\end{array}$ & scale & Length \\
\hline OBJECTID & Object ID & & & & & & \\
\hline Shape & Geometry & Yes & & & & & \\
\hline Shape_Length & Double & Yes & & & 0 & 0 & \\
\hline $\begin{array}{ll}\text { Simple feat } \\
\text { Paved_Are }\end{array}$ & & & & & $\begin{array}{r}\text { Geomet } \\
\text { Contains M value } \\
\text { Contains } Z \text { value }\end{array}$ & 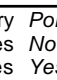 & lygon \\
\hline Field name & Data type & $\begin{array}{l}\text { Allow } \\
\text { nulls }\end{array}$ & Default value & Domain & $\begin{array}{l}\text { Prec- } \\
\text { ision }\end{array}$ & scale & Length \\
\hline OBJECTID & Object ID & & & & & & \\
\hline Shape & Geometry & Yes & & & & & \\
\hline Shape_Length & Double & Yes & & & 0 & 0 & \\
\hline Shape_Area & Double & Yes & & & 0 & 0 & \\
\hline $\begin{array}{ll}\text { Simple feat } \\
\text { Picnic_Tab }\end{array}$ & lass & & & & $\begin{array}{r}\text { Geomet } \\
\text { Contains M value } \\
\text { Contains } Z \text { value }\end{array}$ & $\begin{array}{ll}\text { ry } & P o \\
\text { S No } \\
\text { s } \\
\text { Se. }\end{array}$ & lygon \\
\hline Field name & Data type & $\begin{array}{l}\text { Allow } \\
\text { nulls }\end{array}$ & Default value & Domain & $\begin{array}{l}\text { Prec- } \\
\text { ision }\end{array}$ & scale & Length \\
\hline OBJECTID & Object ID & & & & & & \\
\hline Shape & Geometry & Yes & & & & & \\
\hline Shape_Length & Double & Yes & & & 0 & 0 & \\
\hline Shape_Area & Double & Yes & & & 0 & 0 & \\
\hline $\begin{array}{ll}\text { Simple feat } \\
\text { Planters }\end{array}$ & lass & & & & $\begin{array}{r}\text { Geomet } \\
\text { Contains M valu } \\
\text { Contains } Z \text { value }\end{array}$ & $\begin{array}{ll}\text { ry } & P o \\
\text { s } & N o \\
\text { s } & Y e .\end{array}$ & lygon \\
\hline Field name & Data type & $\begin{array}{l}\text { Allow } \\
\text { nulls }\end{array}$ & Default value & Domain & $\begin{array}{l}\text { Prec- } \\
\text { ision }\end{array}$ & scale & Length \\
\hline OBJECTID & Object ID & & & & & & \\
\hline Shape & Geometry & Yes & & & & & \\
\hline Shape_Length & Double & Yes & & & 0 & 0 & \\
\hline Shape_Area & Double & Yes & & & 0 & 0 & \\
\hline \begin{tabular}{|l|l} 
Simple feat \\
Playgroun
\end{tabular} & lass & & & & $\begin{array}{r}\text { Geomet } \\
\text { Contains M valu } \\
\text { Contains } Z \text { value }\end{array}$ & 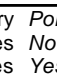 & lygon \\
\hline Field name & Data type & $\begin{array}{l}\text { Allow } \\
\text { nulls }\end{array}$ & Default value & Domain & $\begin{array}{l}\text { Prec- } \\
\text { ision }\end{array}$ & scale & Length \\
\hline OBJECTID & Object ID & & & & & & \\
\hline Shape & Geometry & Yes & & & & & \\
\hline Shape_Length & Double & Yes & & & 0 & 0 & \\
\hline Shape_Area & Double & Yes & & & 0 & 0 & \\
\hline $\begin{array}{ll}\text { Simple feat } \\
\text { Sand_Pit }\end{array}$ & lass & & & & $\begin{array}{r}\text { Geomet } \\
\text { Contains M valu } \\
\text { Contains } Z \text { value }\end{array}$ & $\begin{array}{ll}r y & P o \\
\text { S } & N o \\
\text { S } & Y e\end{array}$ & lygon \\
\hline Field name & Data type & $\begin{array}{l}\text { Allow } \\
\text { nulls }\end{array}$ & Default value & Domain & $\begin{array}{l}\text { Prec- } \\
\text { ision }\end{array}$ & scale & Length \\
\hline OBJECTID & Object ID & & & & & & \\
\hline Shape & Geometry & Yes & & & & & \\
\hline Shape_Length & Double & Yes & & & 0 & 0 & \\
\hline Shape_Area & Double & Yes & & & 0 & 0 & \\
\hline
\end{tabular}




\begin{tabular}{|c|c|c|c|c|c|c|c|}
\hline \multicolumn{4}{|c|}{\begin{tabular}{l|l} 
Simple feature class \\
Shade_Canopy
\end{tabular}} & \multicolumn{4}{|c|}{$\begin{aligned} \text { Geometry } & \text { Polygon } \\
\text { Contains M values No } & \text { No } \\
\text { Contains } Z \text { values } & \text { Yes }\end{aligned}$} \\
\hline Field name & Data type & $\begin{array}{l}\text { Allow } \\
\text { nulls }\end{array}$ & Default value & \multirow[t]{2}{*}{ Domain } & \multicolumn{3}{|c|}{$\begin{array}{l}\text { Prec- } \\
\text { ision Scale Length }\end{array}$} \\
\hline OBJECTID & Object ID & & & & & & \\
\hline Shape & Geometry & Yes & & & & & \\
\hline Shape_Length & Double & Yes & & & 0 & 0 & \\
\hline Shape_Area & Double & Yes & & & 0 & 0 & \\
\hline
\end{tabular}

\begin{tabular}{|c|c|c|c|c|c|c|c|}
\hline \begin{tabular}{|l|l}
-1 & Simple feat \\
Shed
\end{tabular} & lass & & & & $\begin{array}{l}\text { Geom } \\
\text { s M Mal } \\
\text { is } Z \text { val }\end{array}$ & & $\begin{array}{l}\text { llygon } \\
\text { o } \\
\text { es }\end{array}$ \\
\hline Field name & Data type & $\begin{array}{l}\text { Allow } \\
\text { nulls }\end{array}$ & Default value & Domain & \multicolumn{3}{|c|}{$\begin{array}{l}\text { Prec- } \\
\text { ision Scale Length }\end{array}$} \\
\hline OBJECTID & Object ID & & & & & & \\
\hline Shape & Geometry & Yes & & & & & \\
\hline Shape_Length & Double & Yes & & & 0 & 0 & \\
\hline Shape_Area & Double & Yes & & & 0 & 0 & \\
\hline
\end{tabular}

\begin{tabular}{|c|c|c|c|c|c|c|c|}
\hline \multicolumn{5}{|c|}{$\begin{array}{ll}\text { Simple feature class } \\
\text { Sidewalk }\end{array}$} & \multicolumn{3}{|c|}{$\begin{aligned} \text { Geometry } & \text { Polygon } \\
\text { Contains M values } & N o \\
\text { Contains } Z \text { values } & \text { Yes }\end{aligned}$} \\
\hline Field name & Data type & $\begin{array}{l}\text { Allow } \\
\text { nulls }\end{array}$ & Default value & Domain & $\begin{array}{l}\text { Prec- } \\
\text { ision }\end{array}$ & & e Length \\
\hline OBJECTID & Object ID & & & & & & \\
\hline Shape & Geometry & Yes & & & & & \\
\hline Shape_Length & Double & Yes & & & 0 & 0 & \\
\hline Shape_Area & Double & Yes & & & 0 & 0 & \\
\hline
\end{tabular}

\begin{tabular}{|c|c|c|c|c|c|c|c|}
\hline \multicolumn{5}{|c|}{$\begin{array}{l}\text { Simple feature class } \\
\text { SportsField }\end{array}$} & \multicolumn{3}{|c|}{$\begin{aligned} \text { Geometry } & \text { Polygon } \\
\text { Contains M values } & N o \\
\text { Contains Z values } & \text { Yes }\end{aligned}$} \\
\hline Field name & Data type & $\begin{array}{c}\text { Allow } \\
\text { nulls }\end{array}$ & Default value & Domain & $\begin{array}{l}\text { Prec- } \\
\text { ision S }\end{array}$ & & Length \\
\hline OBJECTID & Object ID & & & & & & \\
\hline Shape & Geometry & Yes & & & & & \\
\hline Shape_Length & Double & Yes & & & 0 & 0 & \\
\hline Shape_Area & Double & Yes & & & 0 & 0 & \\
\hline
\end{tabular}

\begin{tabular}{|c|c|c|c|c|c|c|c|}
\hline \multicolumn{5}{|c|}{$\begin{array}{ll}\text { Simple feature class } \\
\text { Track }\end{array}$} & \multicolumn{3}{|c|}{$\begin{aligned} \text { Geometry } & \text { Polygon } \\
\text { Contains M values } & \mathrm{No} \\
\text { Contains } Z \text { values } & \text { Yes }\end{aligned}$} \\
\hline Field name & Data type & $\begin{array}{l}\text { Allow } \\
\text { nulls }\end{array}$ & Default value & Domain & $\begin{array}{l}\text { Prec- } \\
\text { ision }\end{array}$ & & Length \\
\hline OBJECTID & Object ID & & & & & & \\
\hline Shape & Geometry & Yes & & & & & \\
\hline Shape_Length & Double & Yes & & & 0 & 0 & \\
\hline Shape_Area & Double & Yes & & & 0 & 0 & \\
\hline
\end{tabular}

\begin{tabular}{|c|c|c|c|c|c|c|c|}
\hline $\begin{array}{ll}\text { Simple fea } \\
\text { Tree_Canc }\end{array}$ & lass & & & & $\begin{array}{l}\text { Geomet } \\
\text { is M value } \\
\text { is } Z \text { value }\end{array}$ & $\begin{array}{ll}\text { ryy } & P_{0} \\
\text { es } & N c \\
\text { es } & Y_{e}\end{array}$ & $\begin{array}{l}\text { olygon } \\
\text { o } \\
\text { es }\end{array}$ \\
\hline Field name & Data type & $\begin{array}{l}\text { Allow } \\
\text { nulls }\end{array}$ & Default value & Domain & $\begin{array}{l}\text { Prec- } \\
\text { ision }\end{array}$ & Scale & Length \\
\hline OBJECTID & Object ID & & & & & & \\
\hline Shape & Geometry & Yes & & & & & \\
\hline Shape_Length & Double & Yes & & & 0 & 0 & \\
\hline Shape_Area & Double & Yes & & & 0 & 0 & \\
\hline
\end{tabular}

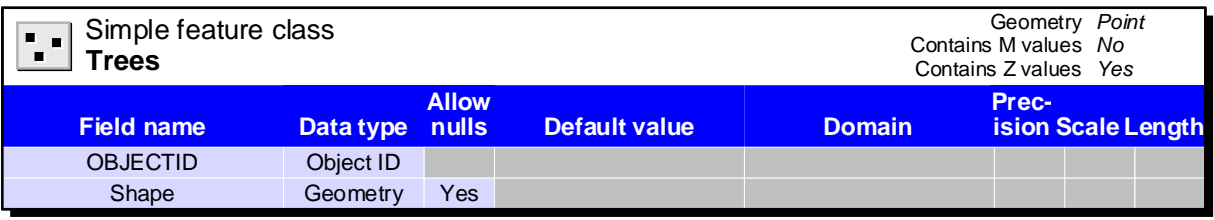

\begin{tabular}{|c|c|c|c|c|c|c|c|}
\hline $\begin{array}{ll}\text { Simple fea } \\
\text { Walkway }\end{array}$ & ass & & & \multicolumn{4}{|c|}{$\begin{aligned} \text { Geometry } & \text { Polygon } \\
\text { Contains M values } & N o \\
\text { Contains Z values } & \text { Yes }\end{aligned}$} \\
\hline Field name & Data type & $\begin{array}{l}\text { Allow } \\
\text { nulls }\end{array}$ & Default value & Domain & $\begin{array}{l}\text { Prec- } \\
\text { ision }\end{array}$ & & e Length \\
\hline OBJECTID & Object ID & & & & & & \\
\hline Shape & Geometry & Yes & & & & & \\
\hline Shape_Length & Double & Yes & & & 0 & 0 & \\
\hline Shape_Area & Double & Yes & & & 0 & 0 & \\
\hline
\end{tabular}




\begin{tabular}{|c|c|c|c|c|c|c|c|}
\hline \multicolumn{4}{|c|}{$\begin{array}{l}\text { - Simple feature class } \\
\text { Inventory_Locations }\end{array}$} & \multicolumn{4}{|c|}{$\begin{aligned} \text { Geometry } & \text { Point } \\
\text { Contains M values } & N o \\
\text { Contains Z values } & \text { Yes }\end{aligned}$} \\
\hline Field name & Data type & $\begin{array}{c}\text { Allow } \\
\text { nulls }\end{array}$ & Default value & \multicolumn{4}{|c|}{$\begin{array}{l}\text { Prec- } \\
\text { ision Scale Length }\end{array}$} \\
\hline OBJECTID & Object ID & & & & & & \\
\hline Shape & Geometry & Yes & & & & & \\
\hline UNIT_ID & String & Yes & & UNIT_ID & & & 255 \\
\hline UNIT_NAME & String & Yes & & & & & 255 \\
\hline LEVEL_ID & String & Yes & NO_VALUE & Level_ID & & & 255 \\
\hline FACILITYYID & String & Yes & NO_VALUE & Facility & & & 255 \\
\hline FACILITY_NAME & String & Yes & NO_VALUE & Facility_name & & & 255 \\
\hline LEVEL_NAME & String & Yes & NO_VALUE & Level_name_short & & & 255 \\
\hline GlobalID & & No & & & 0 & 0 & 38 \\
\hline LOCATION_TYPE & Long integer & Yes & 0 & Location type & 0 & & \\
\hline LOCATION_ID & String & Yes & & & & & 255 \\
\hline $\begin{array}{l}\text { Simple featur } \\
\text { Assets }\end{array}$ & class & & & $\begin{array}{l}\text { Contain } \\
\text { Contair }\end{array}$ & $\begin{array}{l}\text { Geometry } \\
\text { s M values } \\
\text { is } Z \text { values }\end{array}$ & $\begin{array}{l}\text { Poin } \\
\text { No } \\
\text { Yes }\end{array}$ & \\
\hline Field name & Data type & $\begin{array}{c}\text { Allow } \\
\text { nulls }\end{array}$ & Default value & Domain & $\begin{array}{l}\text { Prec- } \\
\text { ision S }\end{array}$ & :ale L & -ength \\
\hline OBJECTID_1 & Object ID & & & & & & \\
\hline Shape & Geometry & Yes & & & & & \\
\hline UNIT_ID & String & Yes & & & & & 255 \\
\hline UNIT_NAME & String & Yes & & & & & 255 \\
\hline LEVEL_ID & String & Yes & & Level_ID & & & 255 \\
\hline LEVEL_NAME & String & Yes & & Level_name_short & & & 255 \\
\hline FACILITY_ID & String & Yes & & Facility & & & 255 \\
\hline FACILITY_NAME & String & Yes & & Facility_name & & & 255 \\
\hline LOCATION_TYPE & Long integer & Yes & & Location type & 0 & & \\
\hline ID & String & Yes & & & & & 255 \\
\hline GlobalID & & No & & & 0 & 0 & 38 \\
\hline CATEGORY & String & Yes & & & & & 255 \\
\hline ITEM_NAME & String & Yes & & & & & 255 \\
\hline PHASE & String & Yes & & & & & 255 \\
\hline QUANTITY & Double & Yes & & & 0 & 0 & \\
\hline QUANTITY_TYPE & String & Yes & & & & & 255 \\
\hline USEFUL_LIFE & Double & Yes & & & 0 & 0 & \\
\hline REMAINING_LIFE & Double & Yes & & & 0 & 0 & \\
\hline $\mathrm{COST}^{-}$ & Double & Yes & & & 0 & 0 & \\
\hline LIABILITY & Double & Yes & & & 0 & 0 & \\
\hline $\begin{array}{l}\text { Simple featur } \\
\text { Doors }\end{array}$ & class & & & $\begin{array}{l}\text { Contain } \\
\text { Contair }\end{array}$ & $\begin{array}{l}\text { Geometry } \\
\text { s M values } \\
\text { is } Z \text { values }\end{array}$ & $\begin{array}{l}\text { Poin } \\
\text { No } \\
\text { Yes }\end{array}$ & \\
\hline Field name & Data type & $\begin{array}{l}\text { Allow } \\
\text { nulls }\end{array}$ & Default value & Domain & $\begin{array}{l}\text { Prec- } \\
\text { ision S }\end{array}$ & gale L & ength \\
\hline OBJECTID & Object ID & & & & & & \\
\hline Shape & Geometry & Yes & & & & & \\
\hline DOOR_NUMBER & String & Yes & & & & & 255 \\
\hline FACILITY_ID & String & Yes & & Facility & & & 255 \\
\hline FACILITY_NAME & String & Yes & & Facility_name & & & 255 \\
\hline LEVEL_ID & String & Yes & & Level_ID & & & 255 \\
\hline LEVEL_NAME & String & Yes & & Level_Name_Short & & & 255 \\
\hline LOCATION_TYPE & String & Yes & & Location Type & & & 255 \\
\hline GlobalID & & No & & & 0 & 0 & 38 \\
\hline
\end{tabular}




\section{Tables}

\begin{tabular}{|c|c|c|c|c|c|c|}
\hline Field name & Data type & $\begin{array}{l}\text { Allow } \\
\text { nulls }\end{array}$ & Default value & Domain & $\begin{array}{l}\text { Prec- } \\
\text { ision: }\end{array}$ & Scale Length \\
\hline OBJECTID & Object ID & & & & & \\
\hline ITEM_NAME & String & Yes & & & & 255 \\
\hline ITEM_TYPE & String & Yes & & Item_type & & 255 \\
\hline QUANTITY & Long integer & Yes & & & 0 & \\
\hline LOCATION_ID & String & Yes & & & & 255 \\
\hline LOCATION_ID_FORM & String & Yes & & & & 255 \\
\hline DESCRIPTION & String & Yes & & & & 255 \\
\hline ITEM_ID & Long integer & Yes & & & 0 & \\
\hline TYPE_OTHER & String & Yes & & & & 255 \\
\hline ITEM_SUBTYPE & String & Yes & & Item_subtype & & 255 \\
\hline
\end{tabular}

\section{Relationship Classes}

\begin{tabular}{|c|c|}
\hline \multicolumn{2}{|l|}{$\begin{array}{l}\square \text { Relationship class } \\
\tau \square \text { Inventory_Rel }\end{array}$} \\
\hline $\begin{array}{l}\quad \text { Type Simple } \\
\text { Cardinality One to many } \\
\text { Notification None }\end{array}$ & $\begin{array}{c}\text { Forward label Inventory } \\
\text { Backward label Inventory_Locatid } \\
\text { ns }\end{array}$ \\
\hline Origin feature class & Destination table \\
\hline $\begin{array}{l}\text { Name Inventory_Locatio } \\
\text { Primary key ns } \\
\text { Foreign key LOCATION_ID } \\
\text { LOCATION ID } \\
\text { NO relationshi }\end{array}$ & hip rules defined. \\
\hline
\end{tabular}




\section{Appendix B. Campus Maps}

\section{Legend}

\section{Facilities}

Management

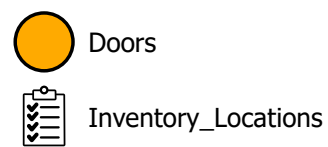

Assets

CATEGORY

(†) electrical

FLOORING

(9) landscape/Hardscape

MeChanical

(○) MiscelLaneous

(ब) PAINT

(7) PLUMBING

ReCreation facilities

$(3$ ROOF/DECKS

8 SPORTS COURT

围) STRUCTURE

\section{Indoors}

— Details

Units

Use Type

\begin{tabular}{l}
\hline Community (Classroom) \\
\hline Electrical \\
\hline Hallway \\
\hline Kitchen \\
\hline Laundry \\
\hline Lobby \\
\hline Lunch Room \\
Multipurpose Room \\
\hline No Value \\
Office \\
\hline Restroom \\
Stairway \\
Storage \\
Workroom \\
Levels
\end{tabular}

\section{Outdoors}

- Trees

- Lightposts

- FireHydrant

- DrinkingFountain

Pathway

Parking_Spaces

Walkway

Tree_Canopy

Track

SportsField

Sidewalk

Shed

Shade_Canopy

Sand_Pit

Playground

Planters

Picnic_Tables

Paved_Area

Parking_Lot

Other

Lawn

Dirt

Creek

Court

Buildings

Bridge

Boundary 


\section{Campus Map Full Extent -- Indoors Level 1}

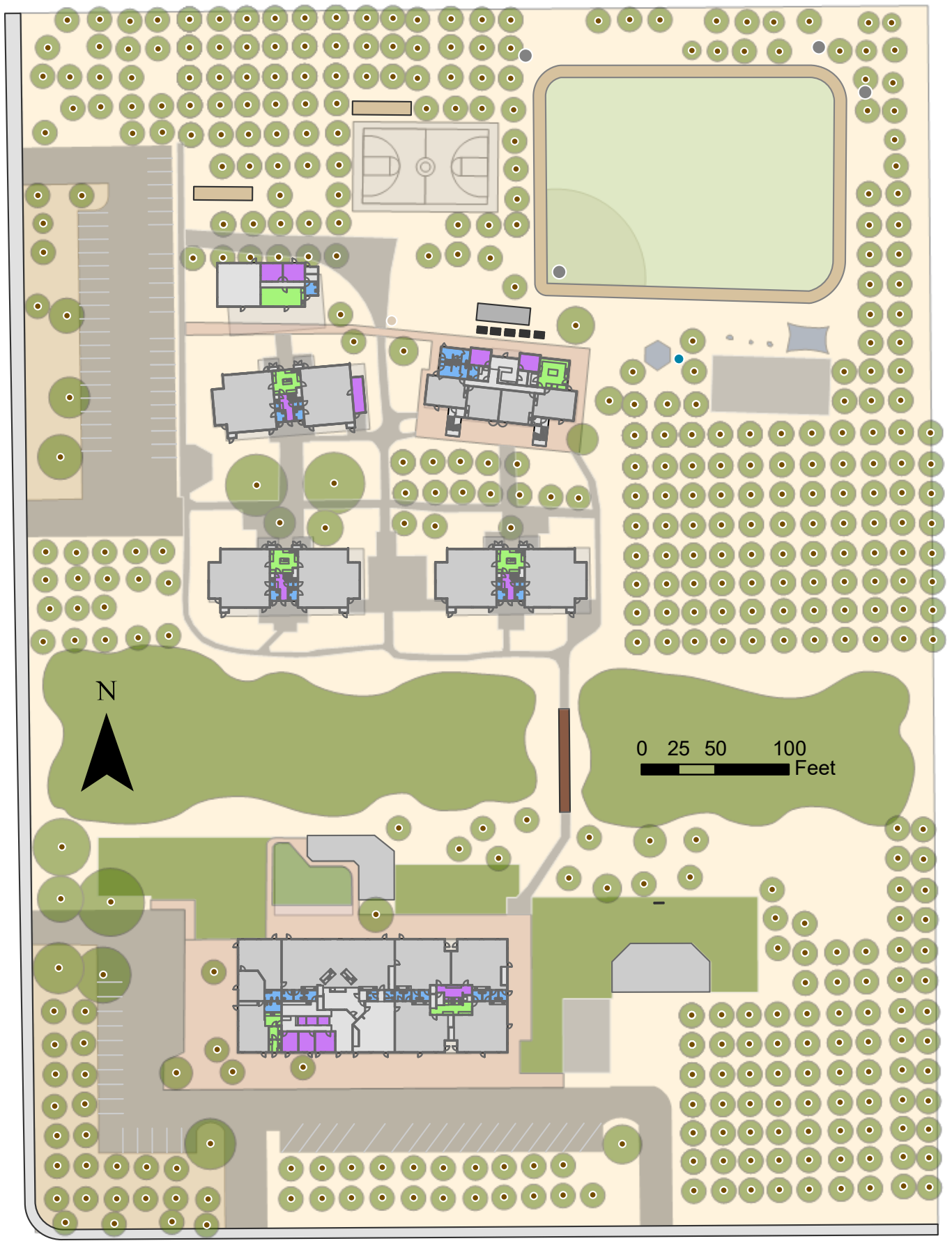




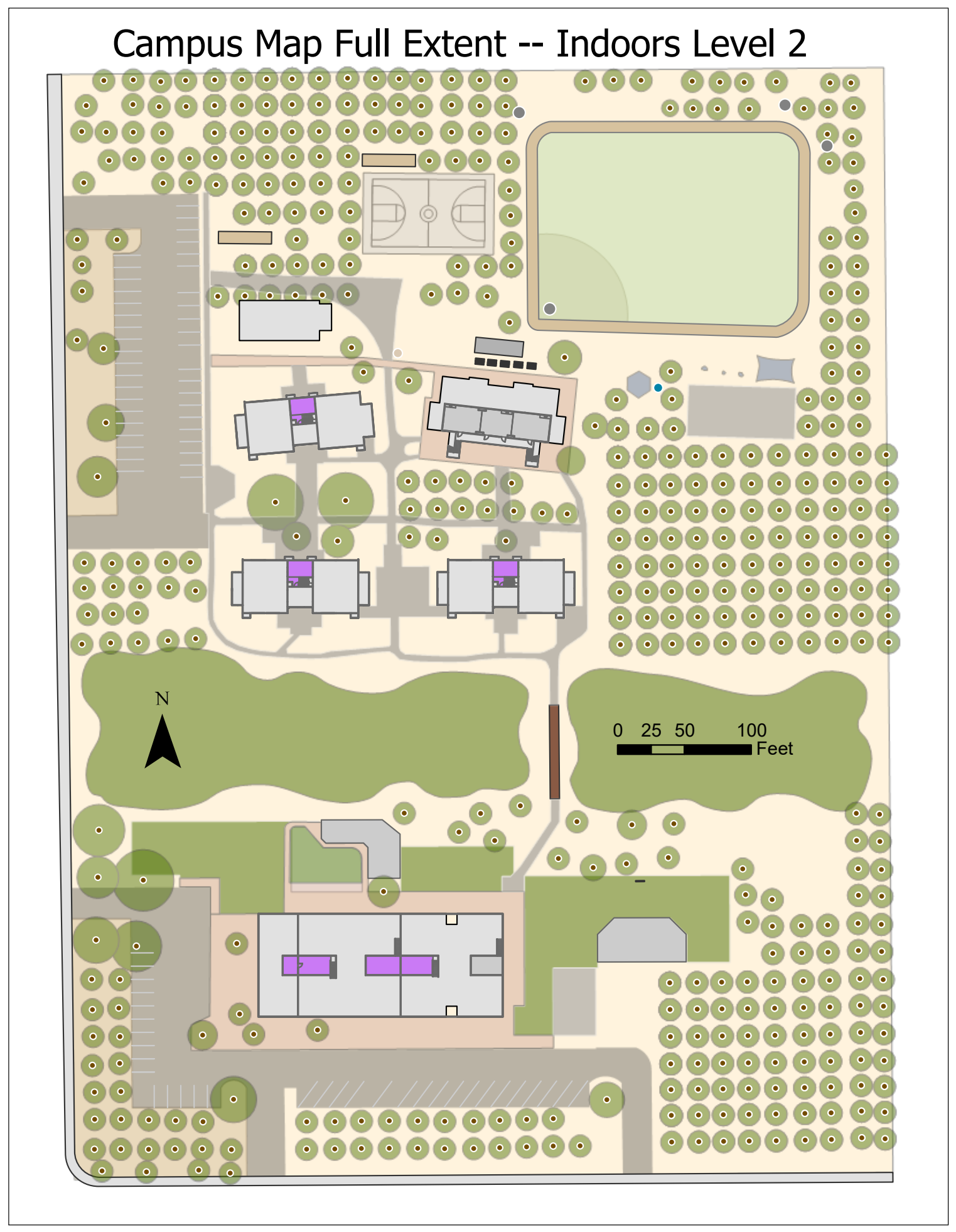




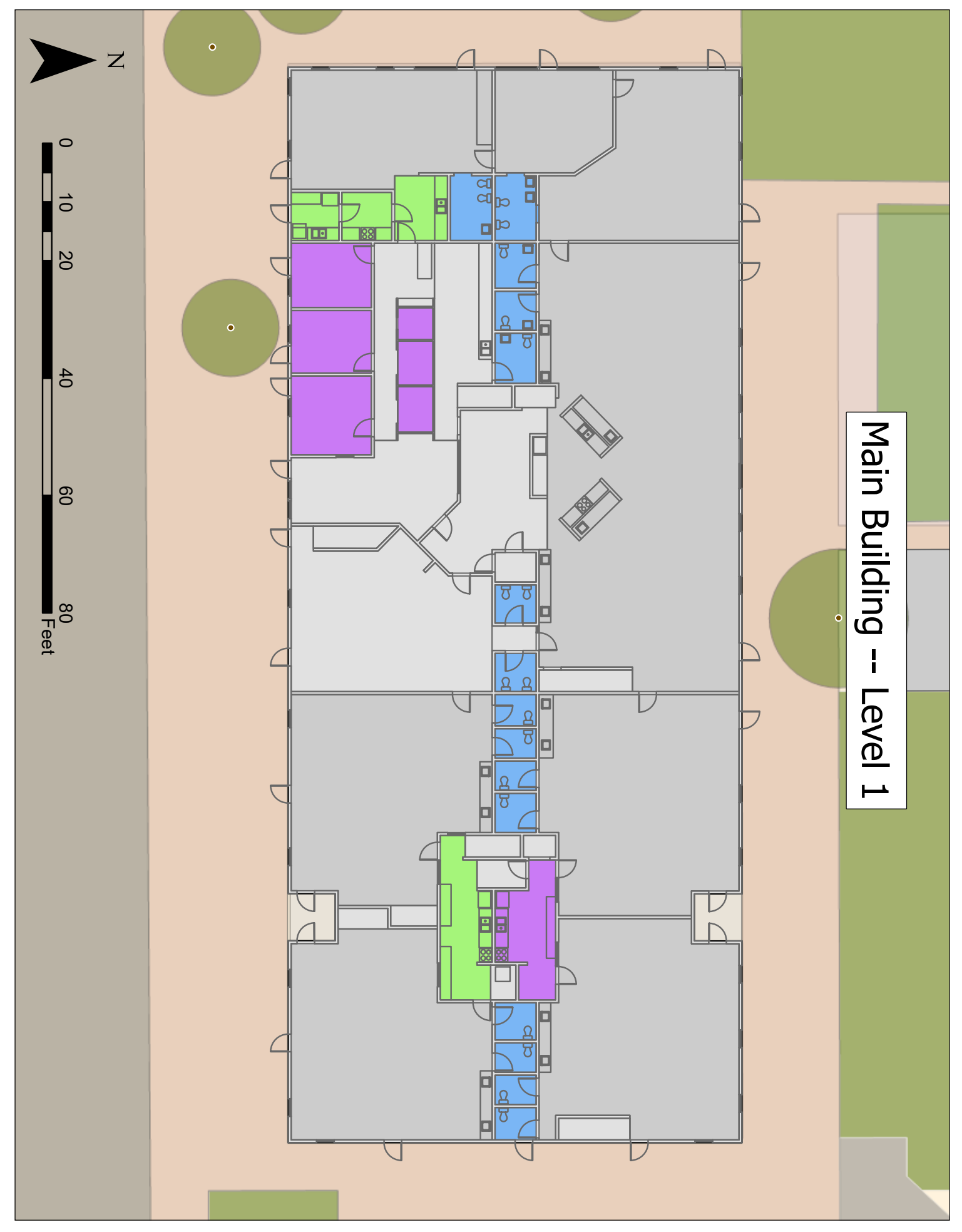




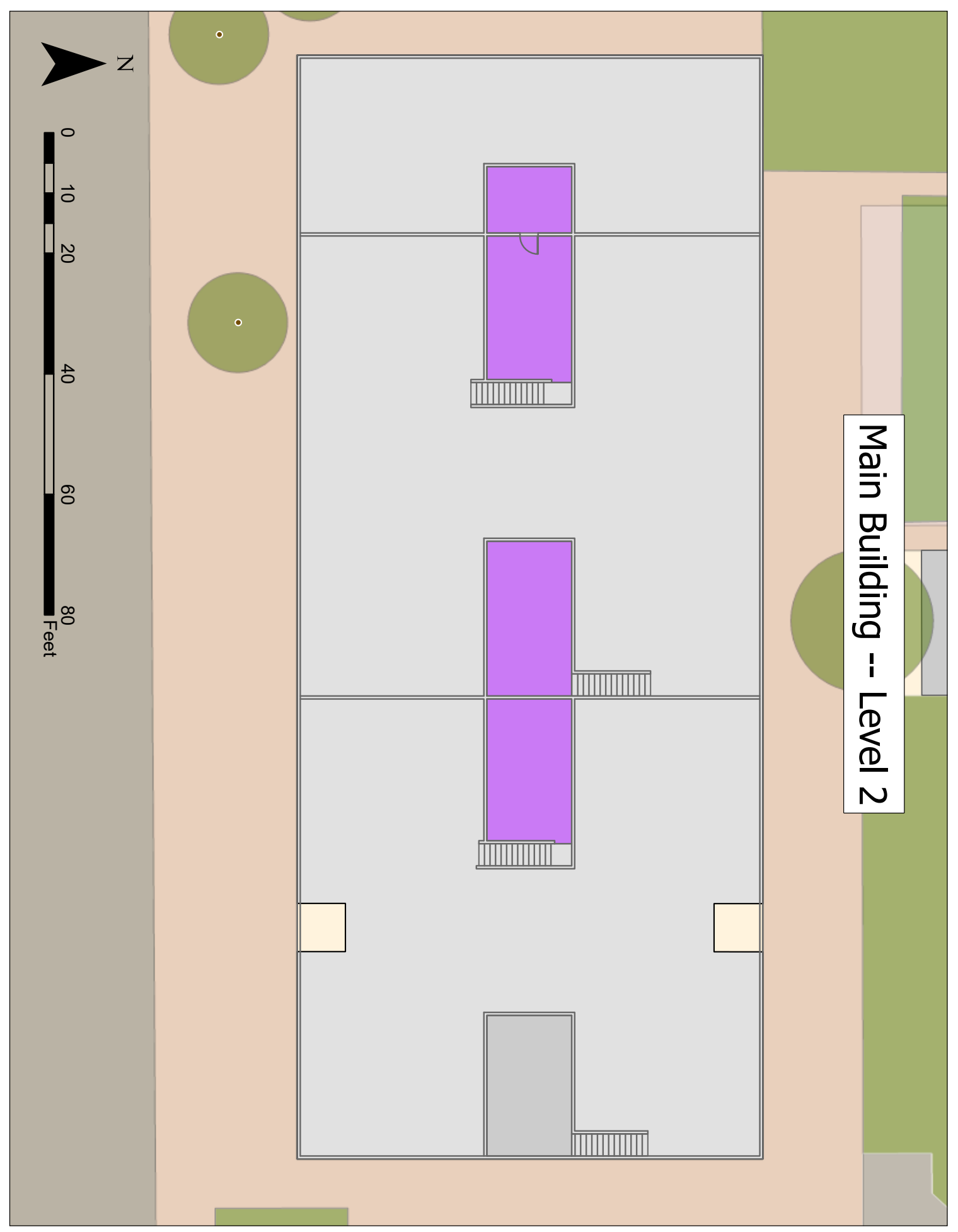




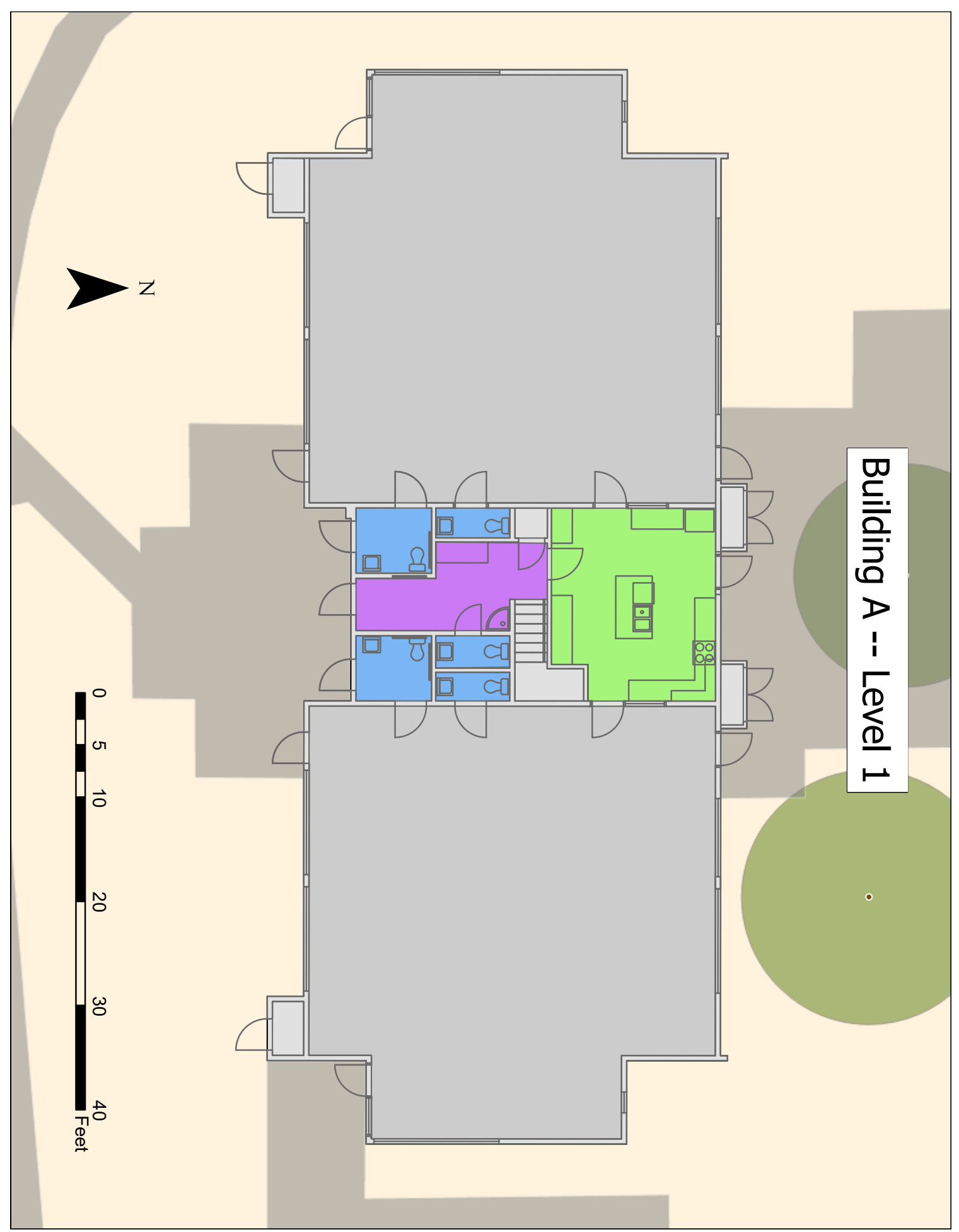




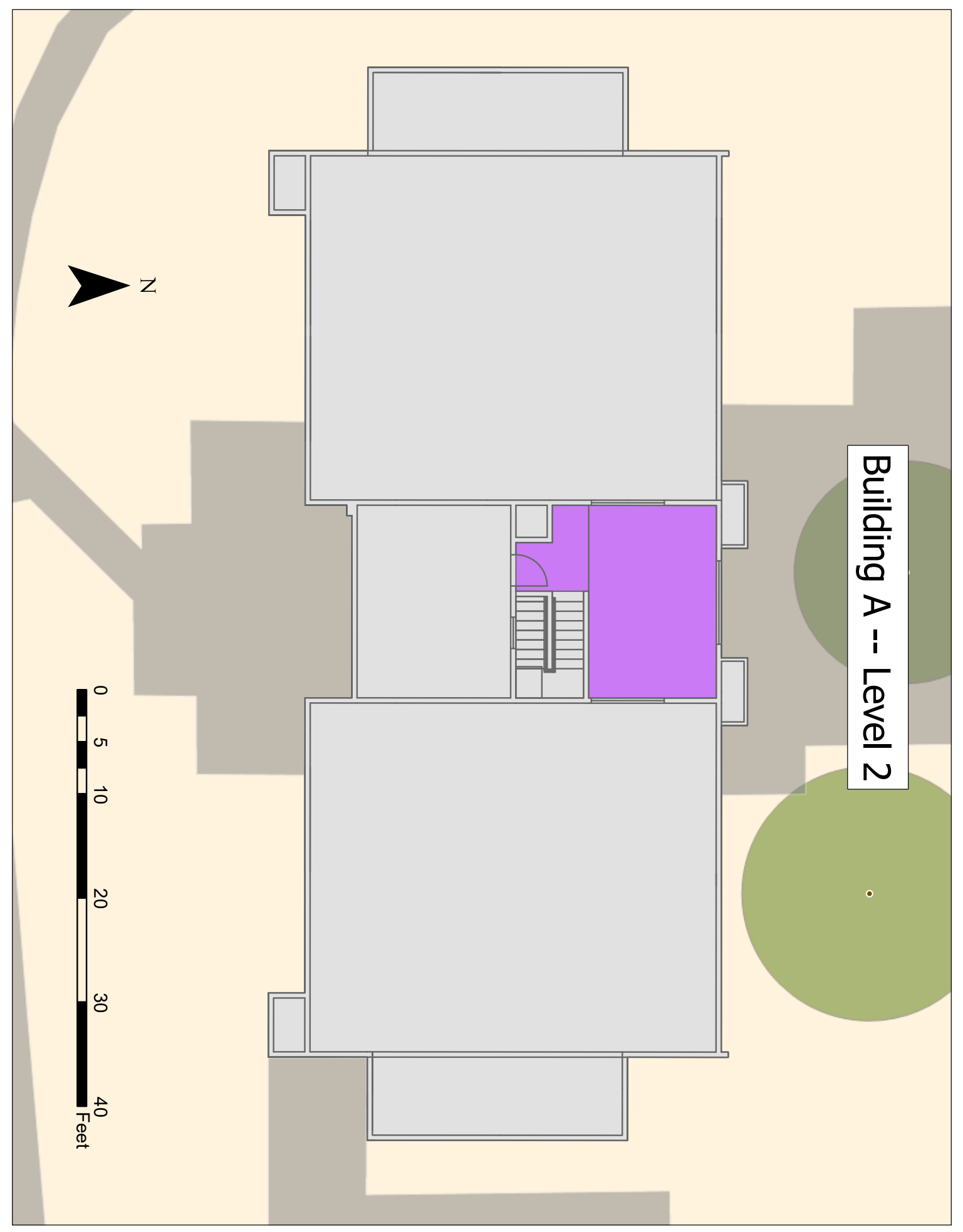




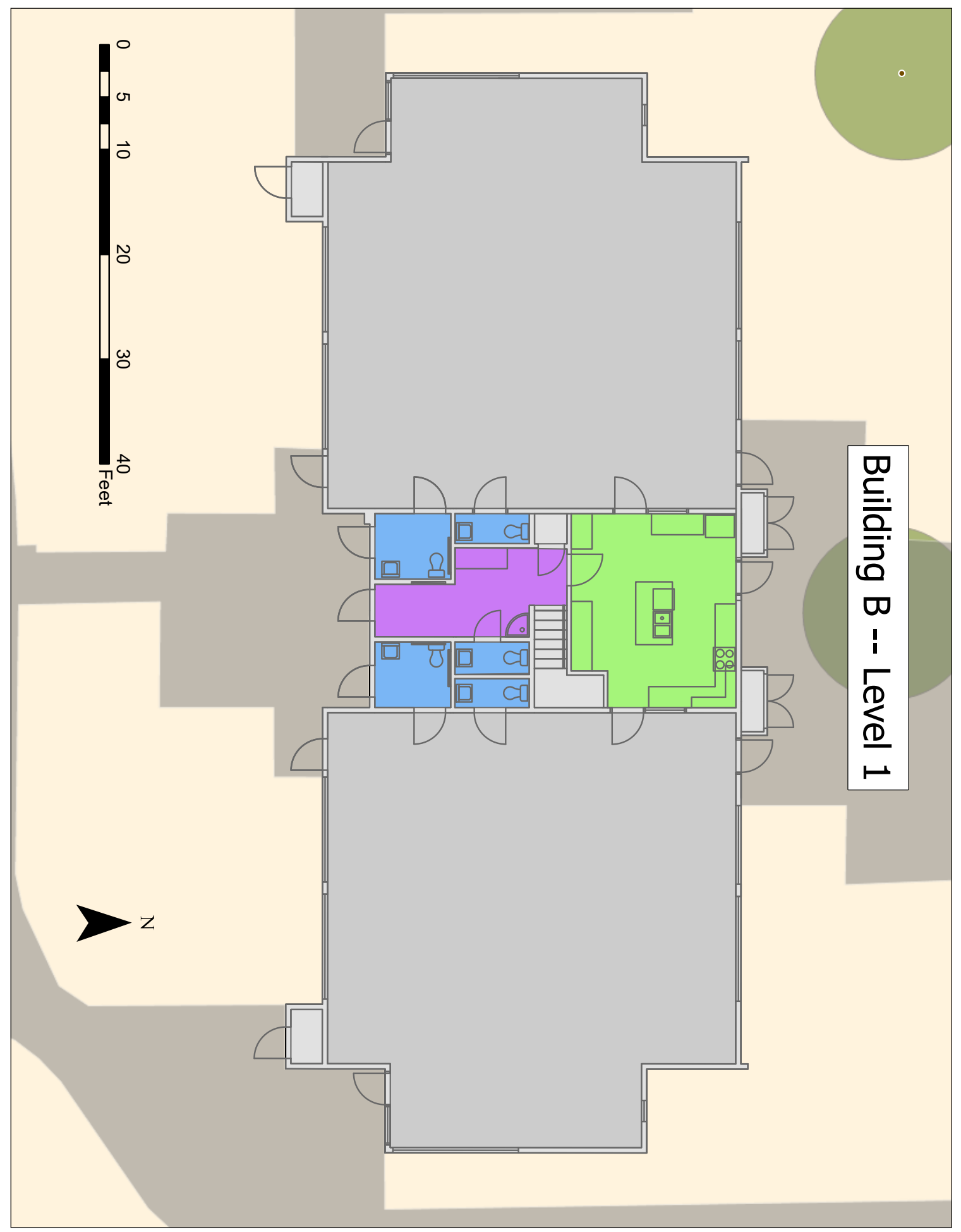




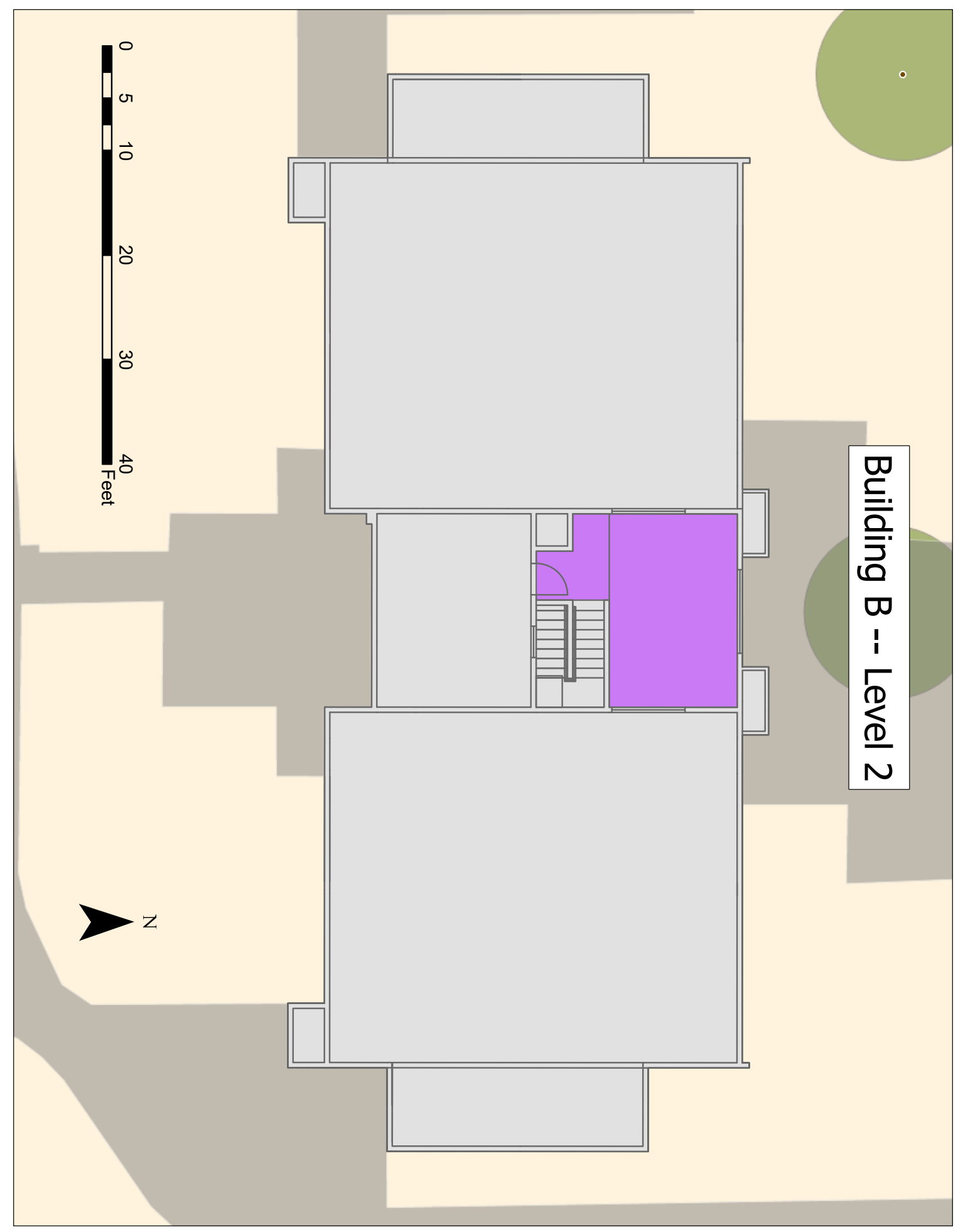




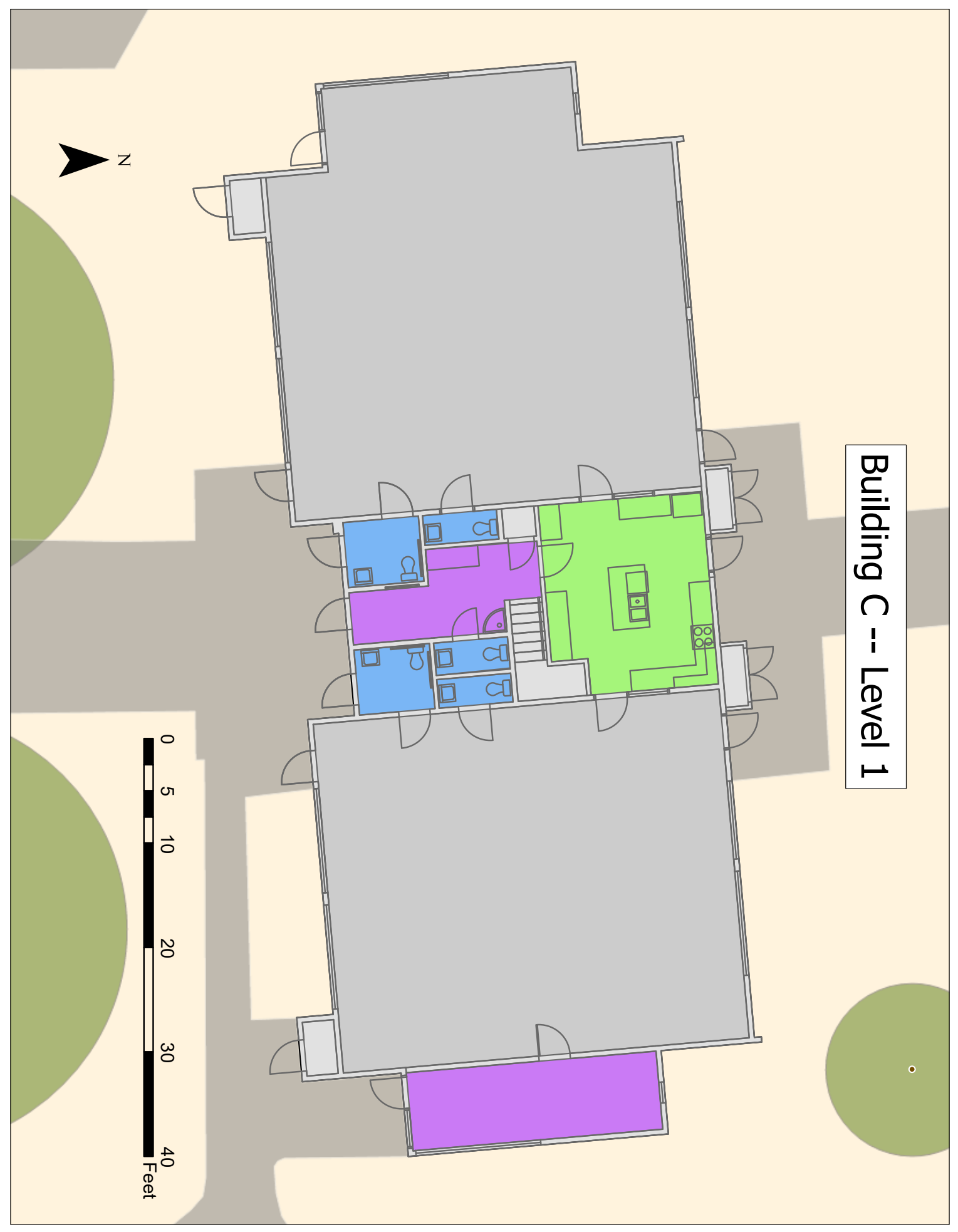




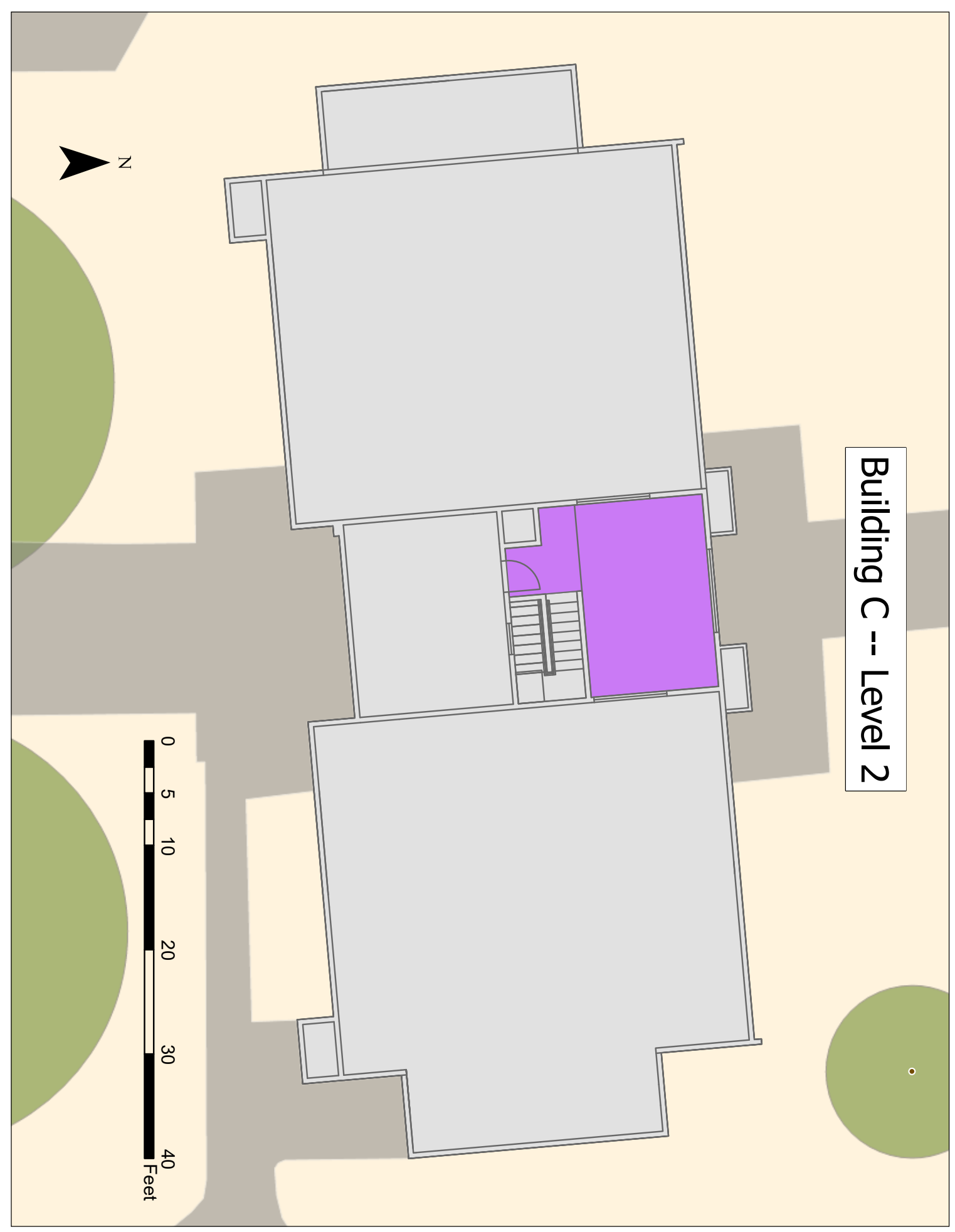




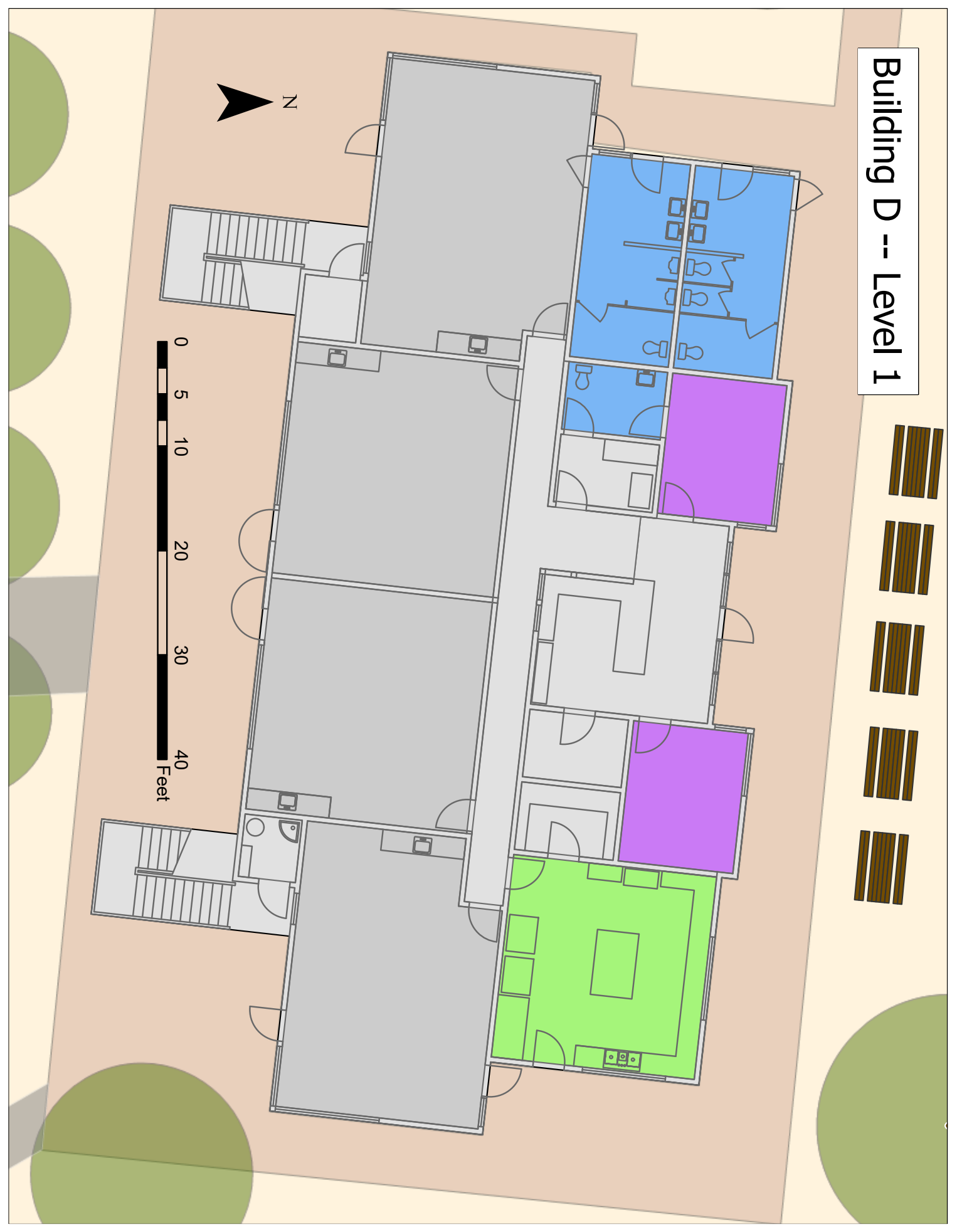




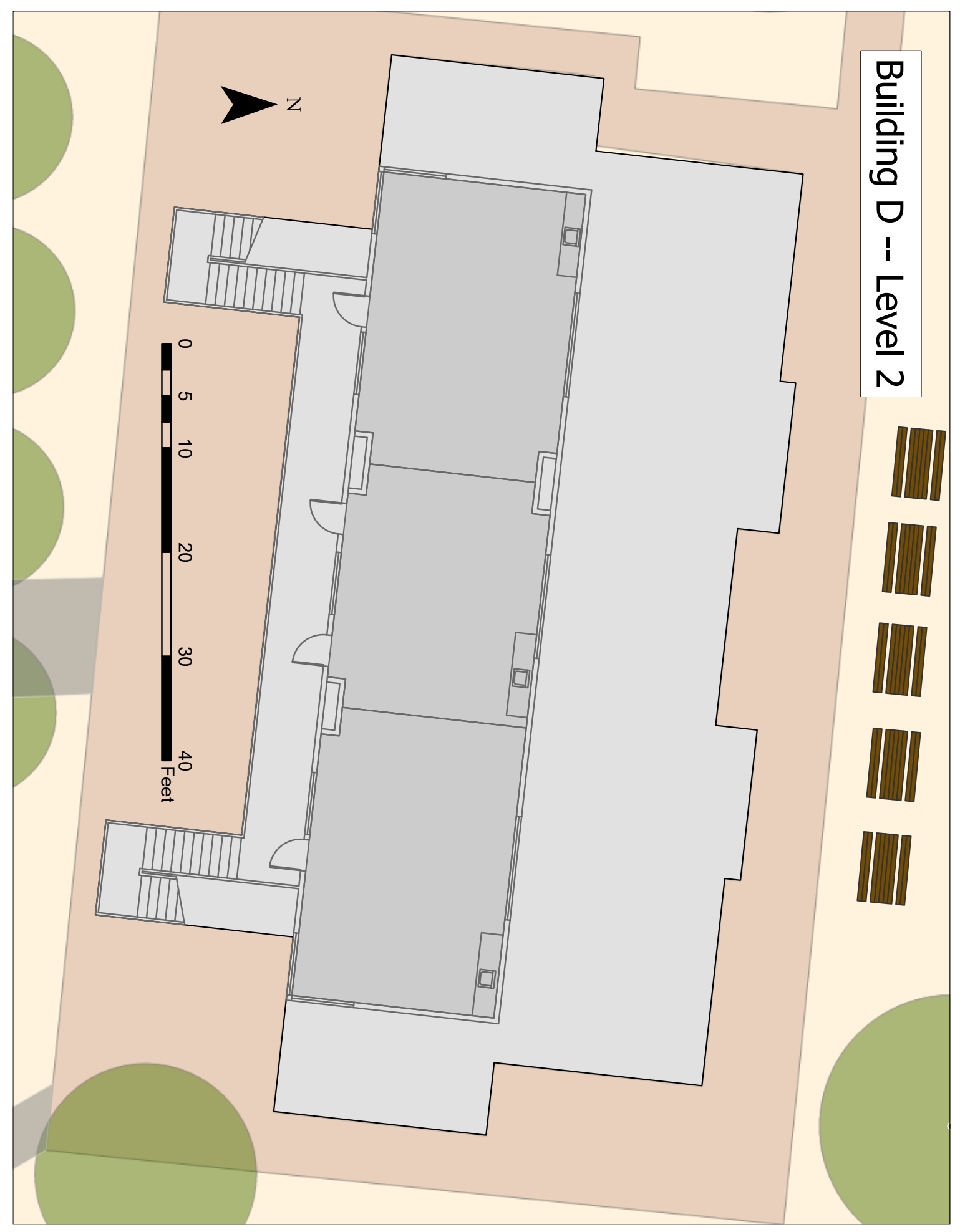




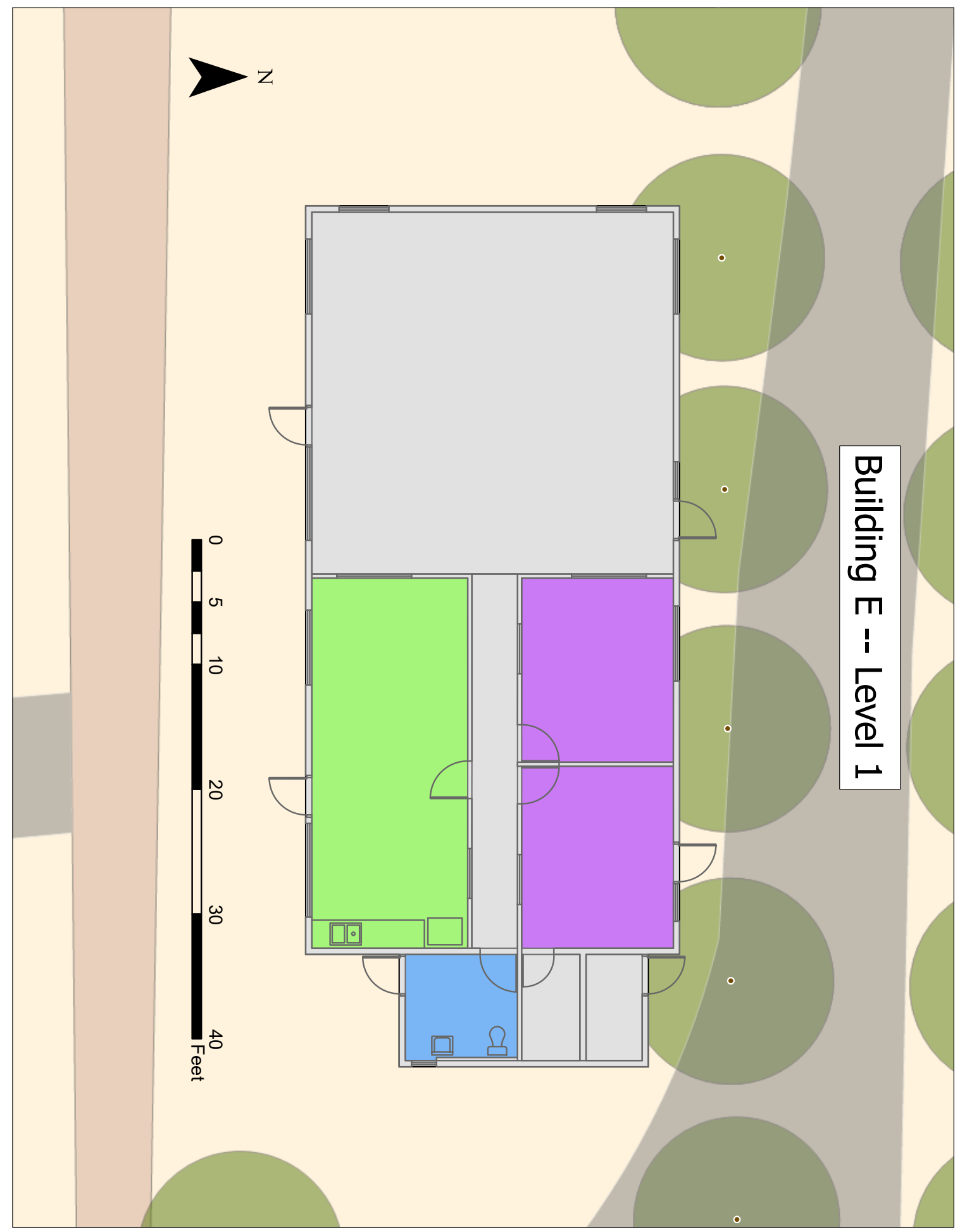




\section{Appendix C. Custom Symbology}

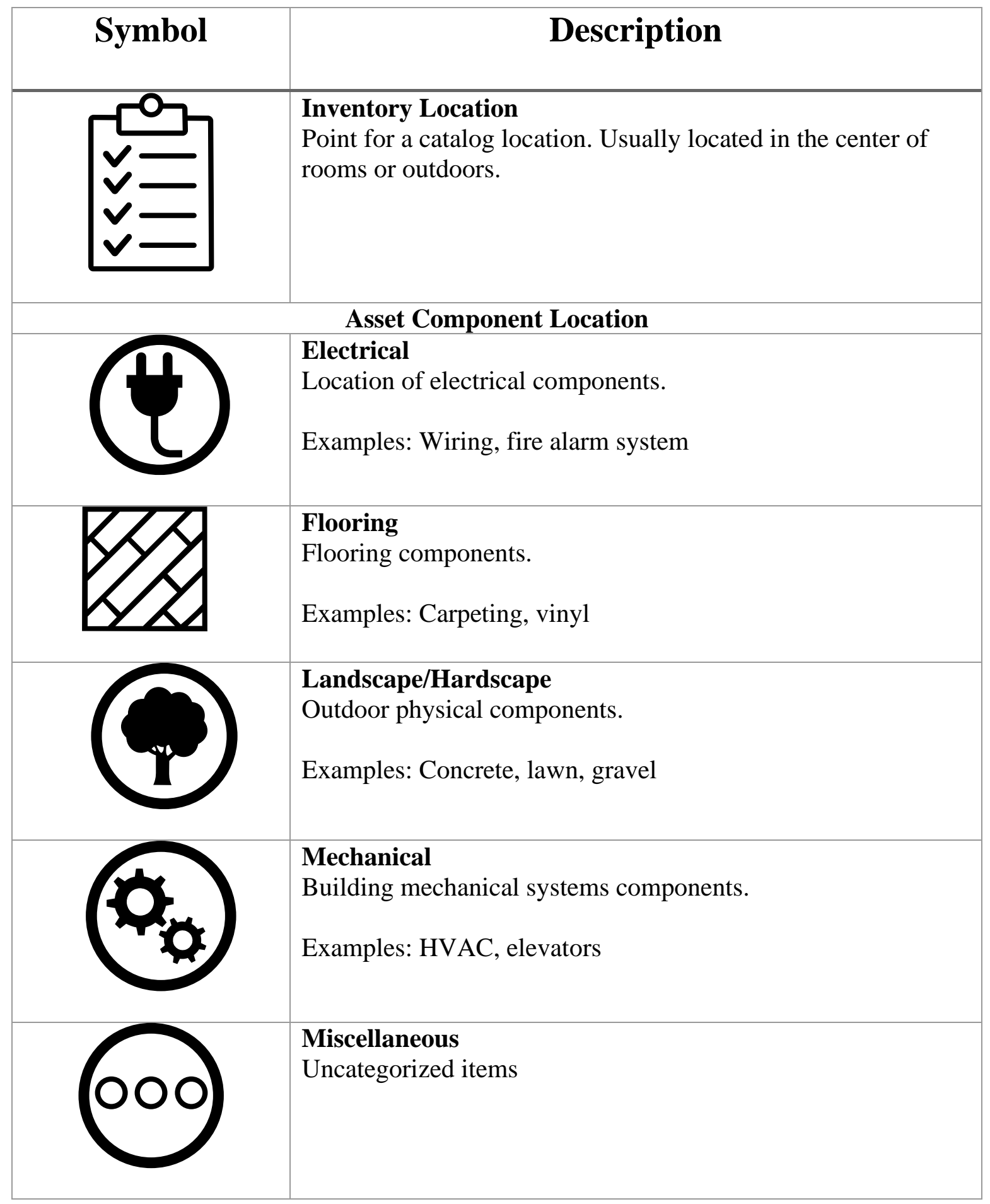




Paint
The painted surface of any other item
Examples: building paint, fencing paint
$\begin{aligned} & \text { Wambing } \\ & \text { Examples: Water heaters, sinks, toilets, piping }\end{aligned}$
$\begin{aligned} & \text { Recreation Facilities } \\ & \text { Eutdoor recreation components. } \\ & \text { Examples: Playground equipment, sports equipment }\end{aligned}$
$\begin{aligned} & \text { Roof/Decks } \\ & \text { Roof and deck components. } \\ & \text { Examples: Roofing, decking, railing } \\ & \text { Examples: Foundation, siding } \\ & \text { Components in sports courts. } \\ & \text { Examples: Playing surface, basketball hoops, backboards }\end{aligned}$




\section{Appendix D. Online System Components}

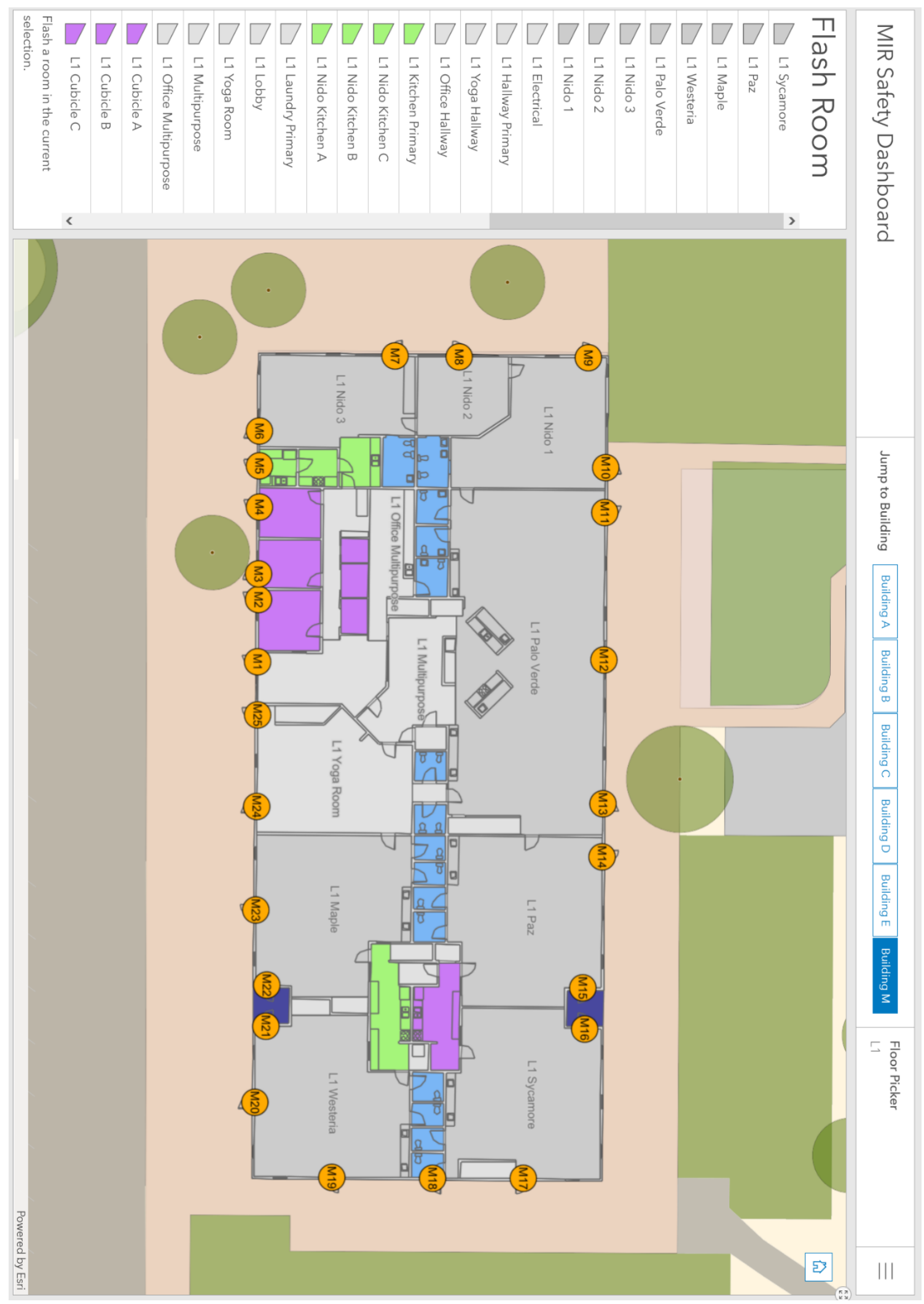




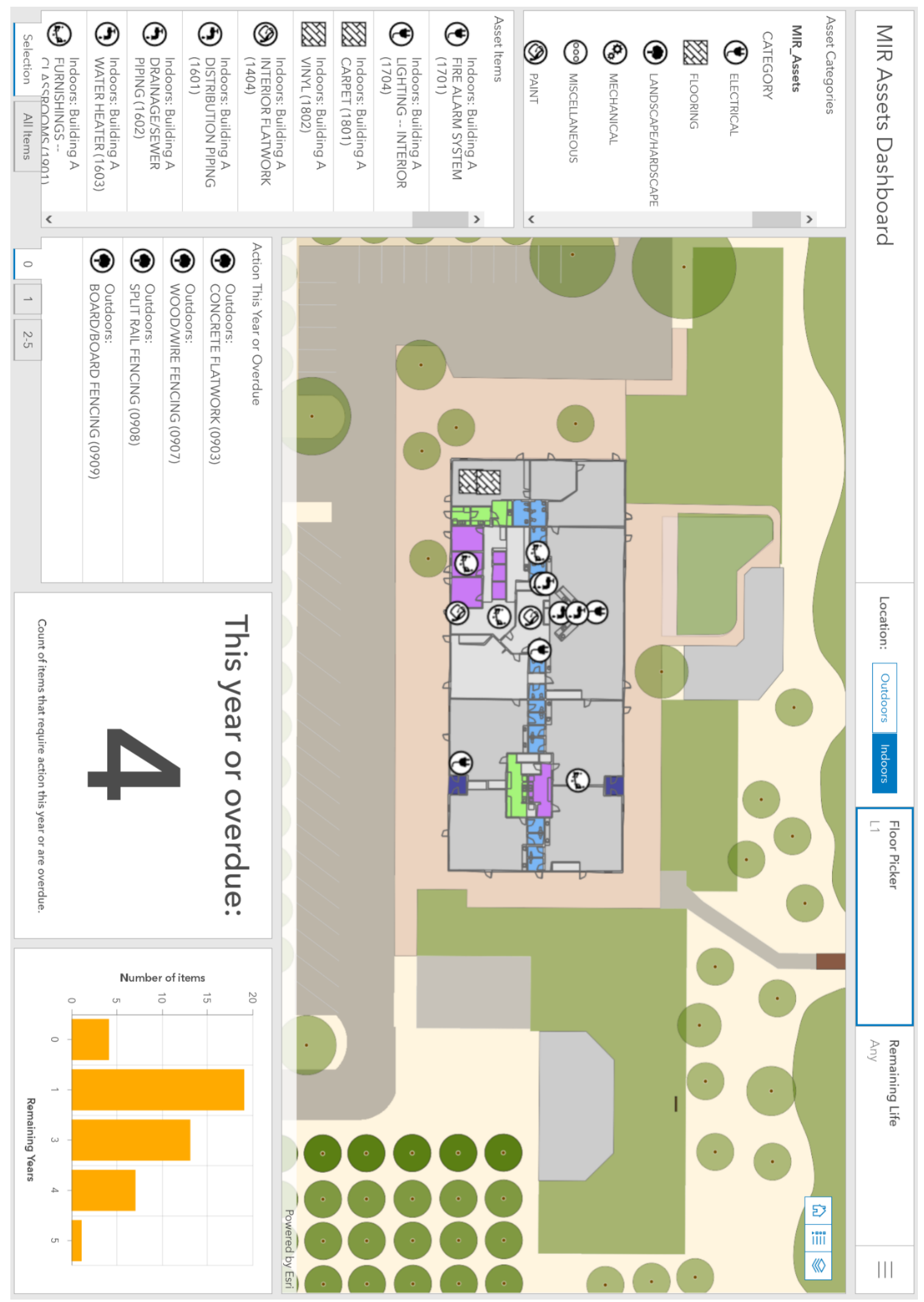




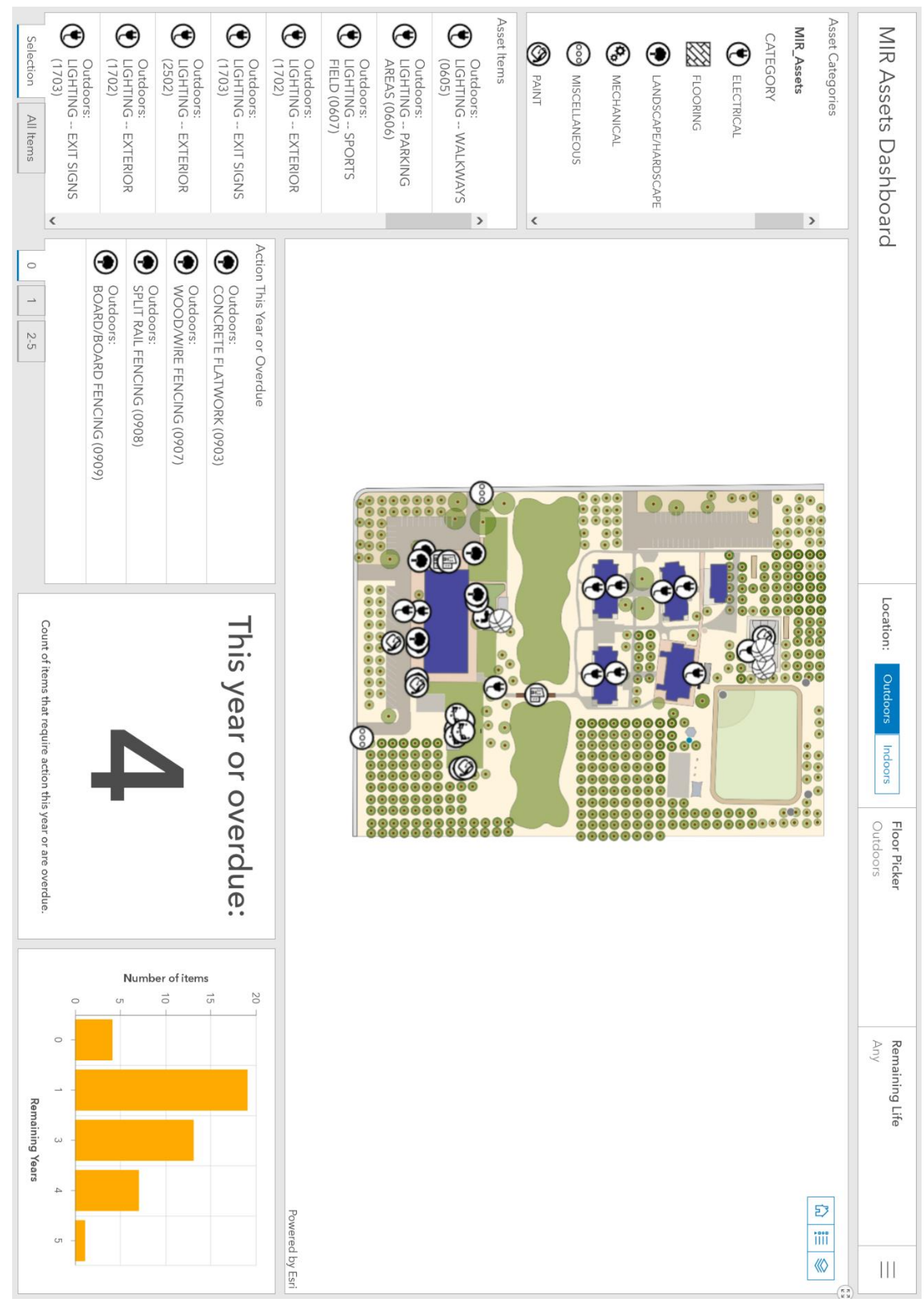




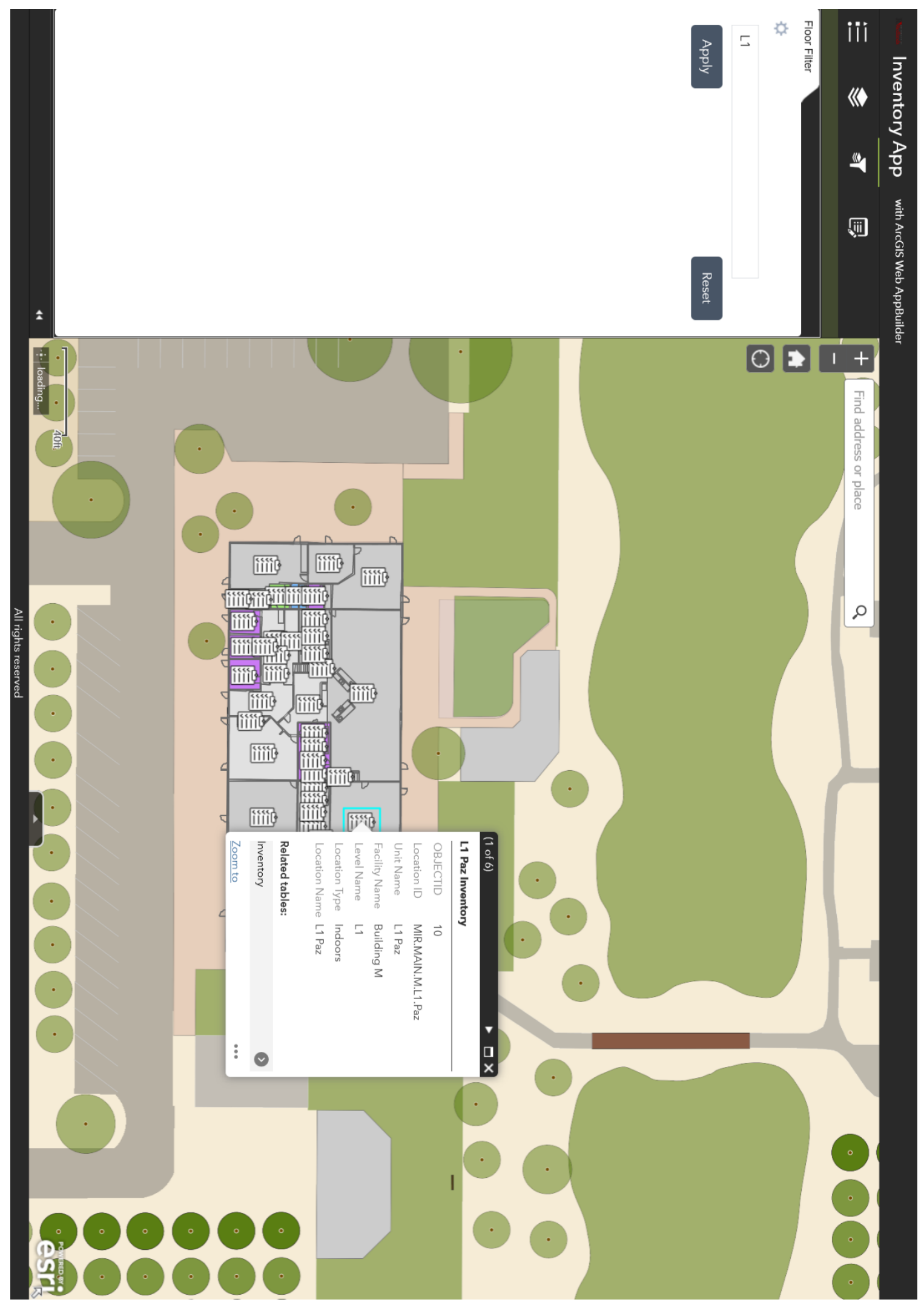




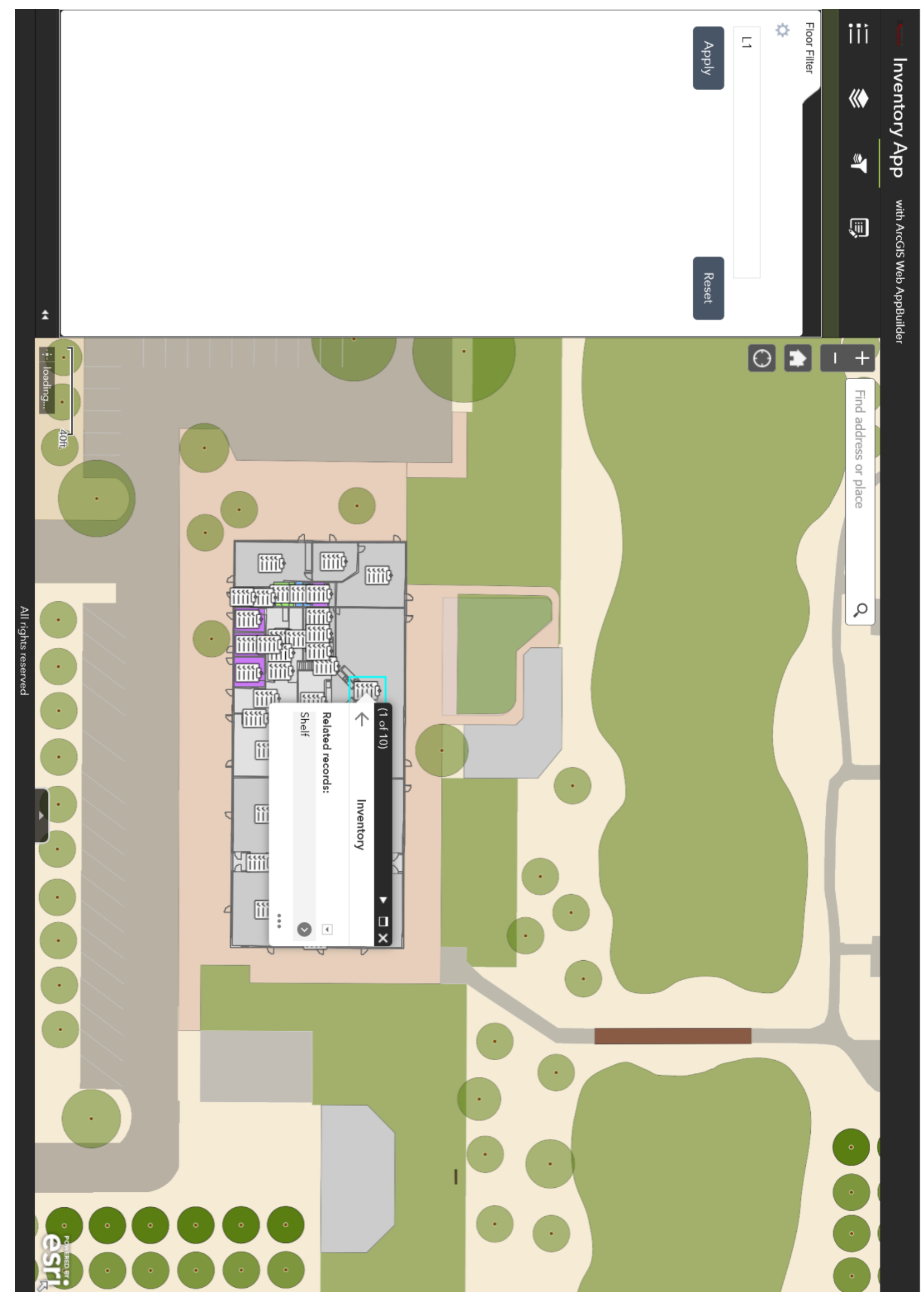




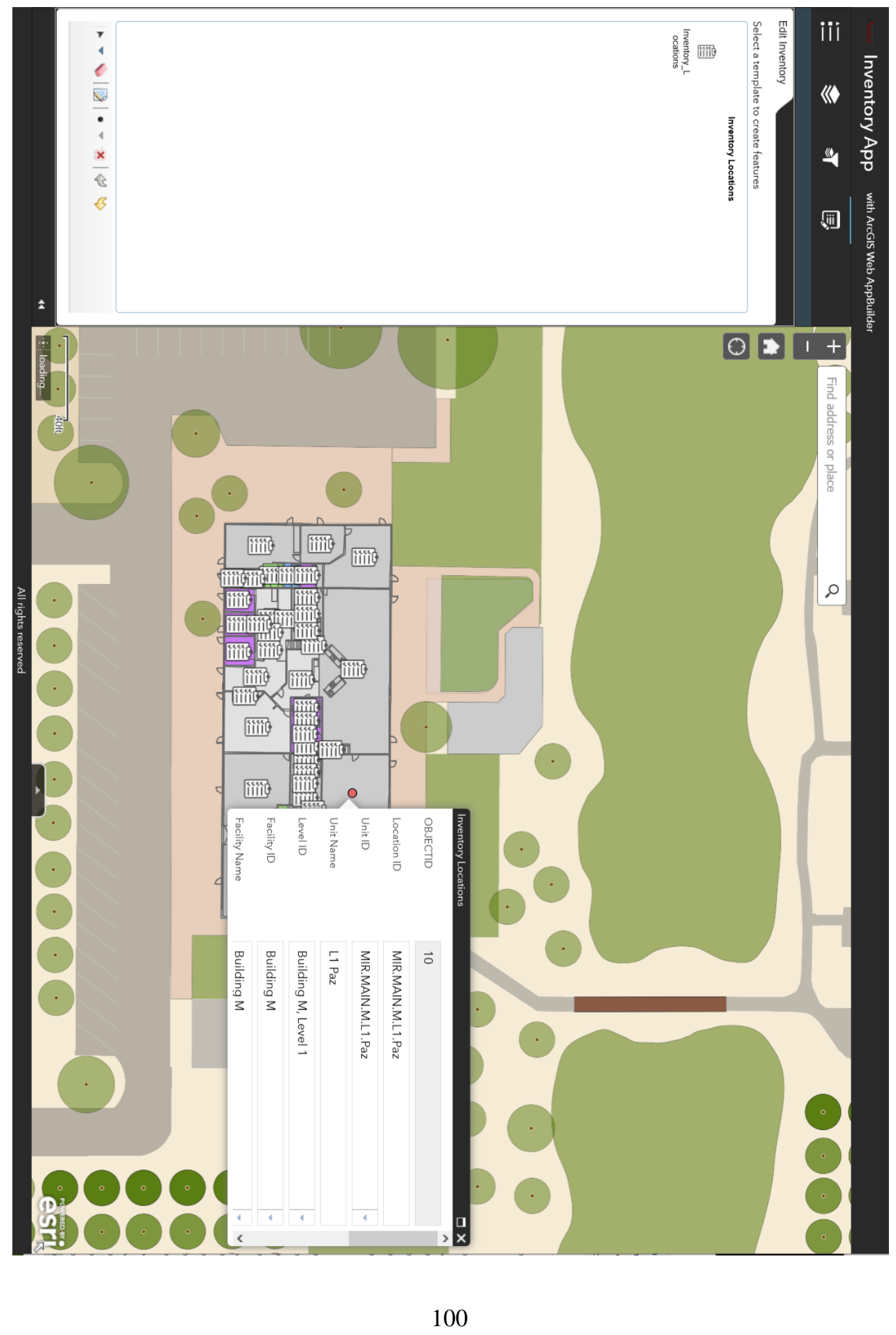




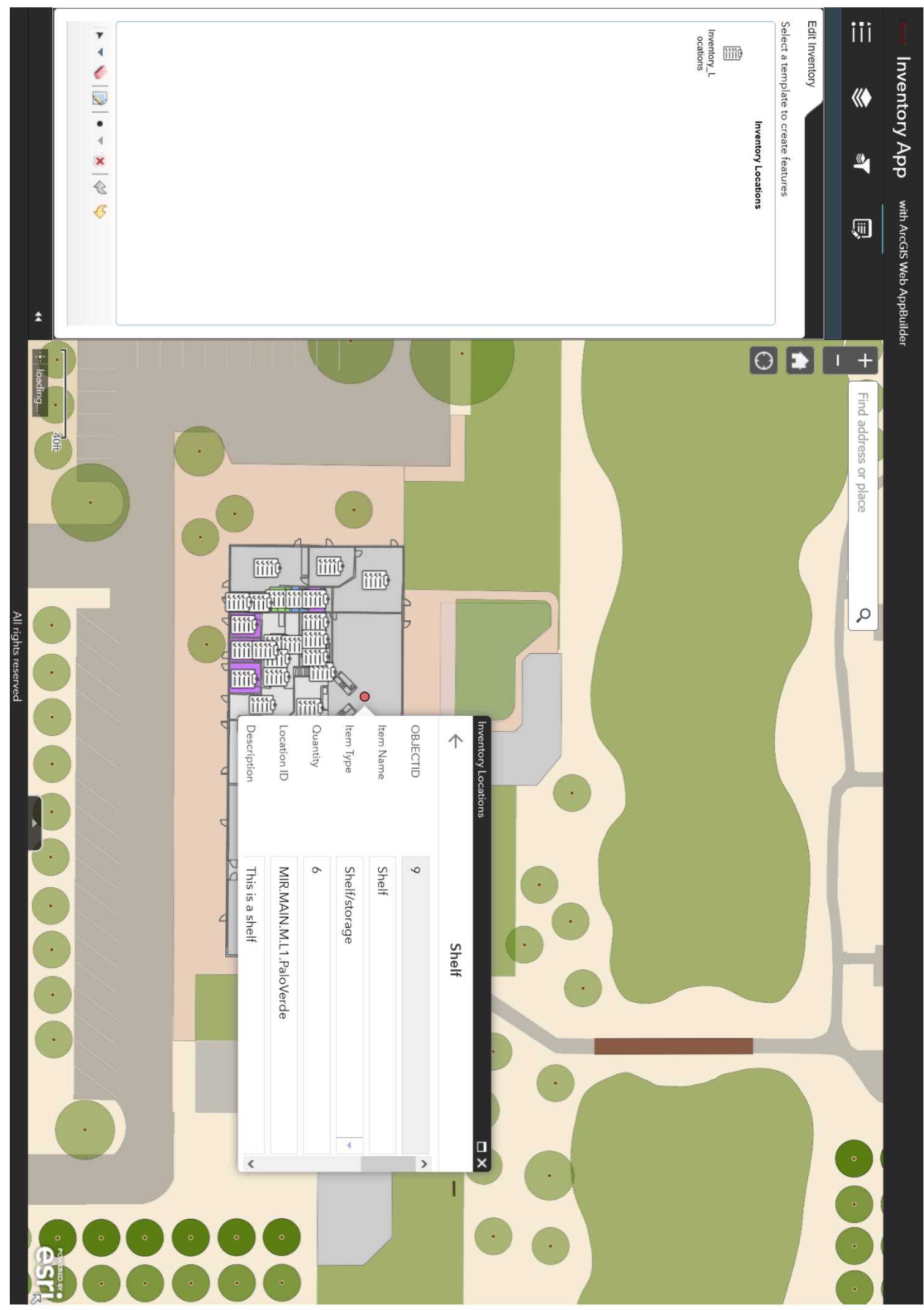

